\title{
Physiology \& pathophysiology of human brown adipose tissue : implications for the treatment of obesity
}

Citation for published version (APA):

Vijgen, G. H. E. J. (2013). Physiology \& pathophysiology of human brown adipose tissue : implications for the treatment of obesity. [Doctoral Thesis, Maastricht University]. Maastricht University. https://doi.org/10.26481/dis.20130607gv

Document status and date:

Published: 01/01/2013

DOI:

10.26481/dis.20130607gv

Document Version:

Publisher's PDF, also known as Version of record

\section{Please check the document version of this publication:}

- A submitted manuscript is the version of the article upon submission and before peer-review. There can be important differences between the submitted version and the official published version of record. People interested in the research are advised to contact the author for the final version of the publication, or visit the DOI to the publisher's website.

- The final author version and the galley proof are versions of the publication after peer review.

- The final published version features the final layout of the paper including the volume, issue and page numbers.

Link to publication

\footnotetext{
General rights rights.

- You may freely distribute the URL identifying the publication in the public portal. please follow below link for the End User Agreement:

www.umlib.nl/taverne-license

Take down policy

If you believe that this document breaches copyright please contact us at:

repository@maastrichtuniversity.nl

providing details and we will investigate your claim.
}

Copyright and moral rights for the publications made accessible in the public portal are retained by the authors and/or other copyright owners and it is a condition of accessing publications that users recognise and abide by the legal requirements associated with these

- Users may download and print one copy of any publication from the public portal for the purpose of private study or research.

- You may not further distribute the material or use it for any profit-making activity or commercial gain

If the publication is distributed under the terms of Article $25 \mathrm{fa}$ of the Dutch Copyright Act, indicated by the "Taverne" license above, 
Physiology \& pathophysiology of human brown adipose tissue Implications for the treatment of obesity

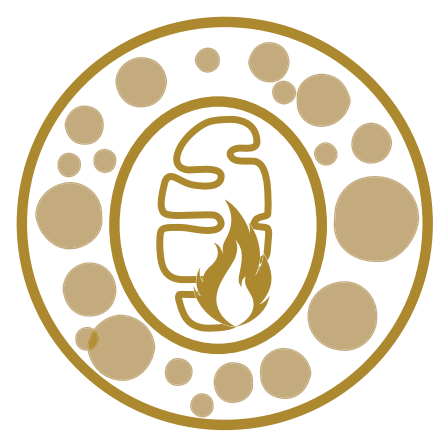

Guy H.E.J. Vijgen 
(C) 2013, Guy H.E.J. Vijgen

Cover design: Max Schulkens

Lay-out: Max Schulkens \& Guy Vijgen

Production: Optima
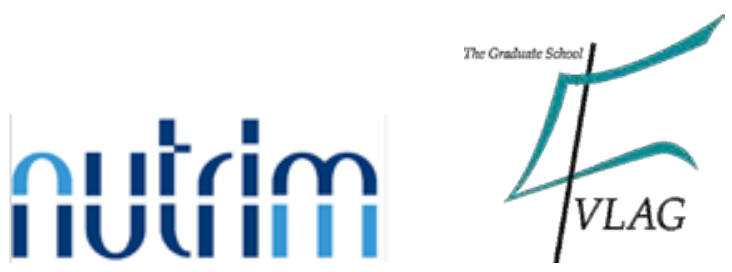

The studies presented in this thesis were performed within NUTRIM School for Nutrition, Toxicology and Metabolism which participates in the Graduate School VLAG, accredited by the Royal Netherlands Academy of Arts and Sciences.

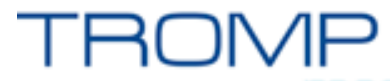

Printing of this thesis was financially supported by Perimed, Covidien, Tromp Medical and the Dutch Society for Metabolic and Bariatric Surgery (DSMBS).

All rights reserved. No part of this thesis may be reproduced or transmitted in any form or by any means, electronic or mechanical, including photocopying, recording or any information storage or retrieval system, without permission in writing from the author, or, when appropriate, from the publishers of the publications. 


\title{
Physiology \& pathophysiology of human brown adipose tissue
}

\author{
Implications for the treatment of obesity
}

\author{
PROEFSCHRIFT \\ Ter verkrijging van de graad van doctor \\ aan de Universiteit Maastricht, \\ op gezag van de Rector Magnificus, \\ Prof. dr. L.L.G. Soete, \\ volgens het besluit van het College der Decanen, \\ in het openbaar te verdedigen \\ op vrijdag 7 juni 2013 om 12.00 uur \\ door \\ Guy Hubertus Elisabeth Joseph Vijgen
}

Geboren op 13 juni 1985 te Geleen, Nederland 


\section{Promotor}

Prof. dr. P. Schrauwen

\section{Co-promotores}

Dr. W.D. van Marken Lichtenbelt

Dr. N.D. Bouvy

\section{Beoordelingscommissie}

Prof. dr. M. van Baak (voorzitter)

Prof. dr. H.A.M. Daanen (Vrije Universiteit Amsterdam / TNO Soesterberg)

Prof. dr. M. Hesselink

Prof. dr. F. Mottaghy

Dr. K.A. Virtanen (University of Turku, Finland) 


\section{Table of Contents}

Chapter $1 \quad$ General Introduction 7

Chapter $2 \quad$ Brown adipose tissue: clinical impact of 21

a re-discovered thermogenic organ

Chapter $3 \quad$ Brown adipose tissue in morbidly obese subjects 45

Chapter $4 \quad$ Increase in brown adipose tissue activity after

61

weight loss in morbidly obese subjects

Chapter $5 \quad$ Increased skeletal muscle mitochondrial function 75

in morbidly obese subjects one year after

bariatric surgery

Chapter 6 Increased oxygen consumption in human

87

adipose tissue from the brown adipose tissue

region

Chapter $7 \quad$ Vagus nerve stimulated increased energy

expenditure in relation to brown adipose

tissue activity

Chapter $8 \quad$ General Discussion

125

Summary

Nederlandse samenvatting (summary in Dutch)

Dankwoord

Scientific output 


$$
\theta_{-1}
$$


Chapter 1

General Introduction 


\section{Brown adipose tissue}

Brown adipose tissue (BAT) has been known since 1551 when the Swiss biologist Conrad Gesner described the observation of a body part in marmots he defined as "neither fat, nor flesh; nec pinguitudo, nec caro" (Figure 1).1,2 This description then already assumed that BAT was different from white adipose tissue (WAT) based on its macroscopic appearance. Throughout the centuries more knowledge has been obtained on BAT and in 1972 a detailed anatomical description of BAT in man was published. ${ }^{3}$ The brown adipocyte (fat cell) contains multiple fat vacuoles and a granular cytoplasm consisting of a large number of mitochondria which gives it its brown appearance. ${ }^{3}$ Except from colour (which varies from caramel-coloured - to brown - to dark red), BAT is highly vascularized, sympathetically innervated and most importantly functionally different from WAT; animal studies have confirmed that the primary function of BAT is heat production upon cold exposure and diet, in contrast to WAT that mainly serves energy storage. ${ }^{4,5}$

\section{Function of brown adipose tissue}

When exposed to a cold environment, the heat production in BAT serves an important function in small animals and human newborns by preventing hypothermia. ${ }^{5}$ This heat production during cold exposure is defined as non-shivering thermogenesis (NST), in contrast to shivering thermogenesis (ST) resulting from skeletal muscle contractions. ${ }^{6}$ NST in BAT is suggested to be responsible for an increase in energy expenditure of $\sim 50 \%$ in mice. ${ }^{5,7}$ In addition to NST, BAT has been shown to become active in rodents after overeating (facultative diet-induced thermogenesis; fDIT). The function of fDIT is to decrease metabolic efficiency and prevent obesity, although this is disputed by others. ${ }^{8,9}$ Genetically modified mice without functional BAT become obese and develop obesity-related comorbidities, suggesting BAT is important in weight management. ${ }^{7}$ This indicates that BAT is an important organ with a vital role in maintaining the energy balance, at least in mice. BAT produces heat by the activity of uncoupling protein 1 (UCP1), first described in $1978 .{ }^{10}$ UCP1 is located on the inner mitochondrial membrane of the brown adipocyte and is capable of producing heat. ${ }^{5,11}$ In the majority of the cells in the human body, the mitochondrial proton gradient is designed to produce adenosine-triphosphate (ATP), the key energy carrier in all of the human body's metabolic processes. However, in BAT the unique presence of UCP1 facilitates proton leakage over the mitochondrial inner-membrane and thus heat production instead of ATP generation. 

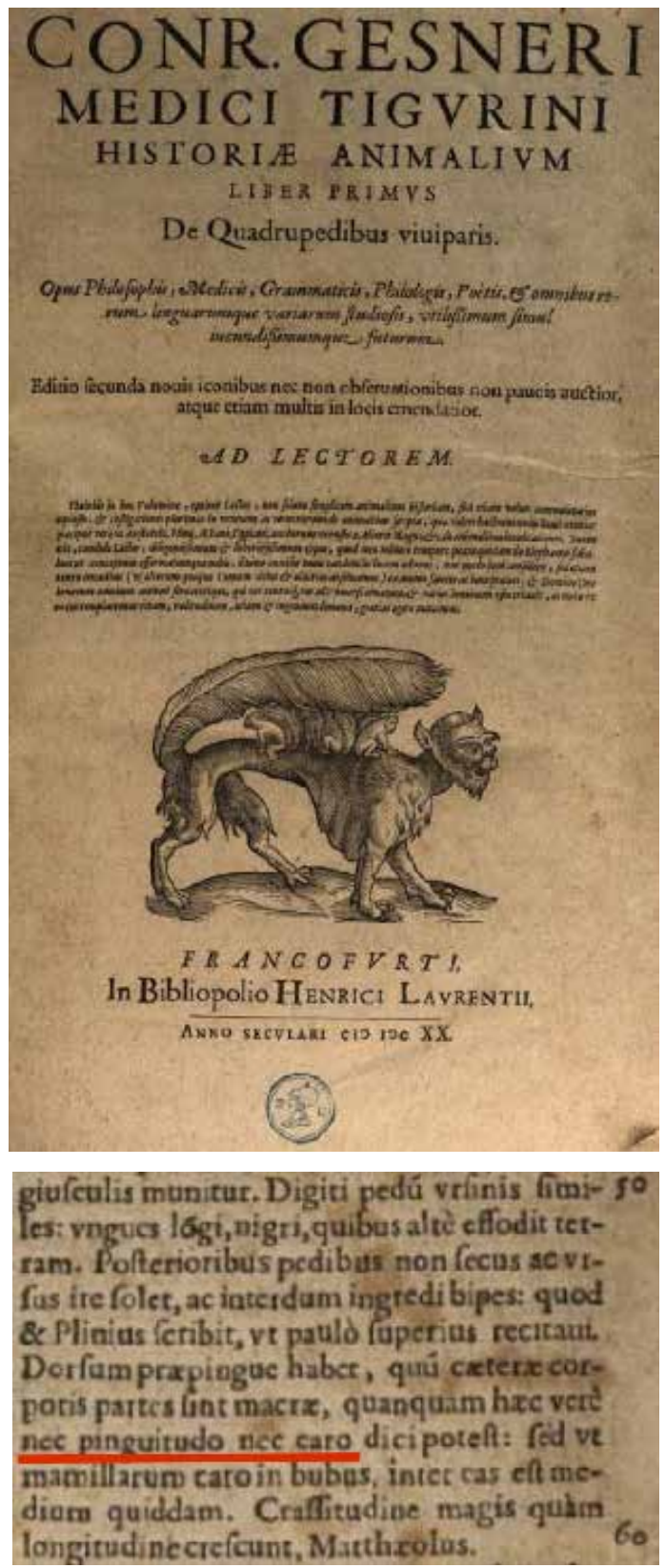

Figure 1. Front of Conrad Gesner's Historiae Animalum, with underlined detail of the first description of brown adipose tissue; "nec pinguitido, nec caro", neither fat nor flesh. Figure obtained from Google Books, with special thanks to prof. Jan Nedergaard. 


\section{Rediscovery of functional brown adipose tissue in adult humans}

In human neonates, BAT is found in the neck along the greater vessels and muscles, supraclavicular and interscapular area, mediastinum and around the aorta, pancreas, spleen, kidneys and suprarenal glands. ${ }^{3}$ The total weight of BAT ( 150-250 g) in newborns consists of 2-5\% of the body's total amount of adipose tissue. ${ }^{4}$ BAT in adults was known for several decades, but it has only recently been shown that adult humans possess significant amounts of physiological active BAT. In 1985 Astrup et al. performed dedicated studies on human BAT and measured temperatures in interscapular and perirenal BAT before and after stimulation with the sympathomimetic ephedrine.12,13 Although thermogenesis increased significantly, unfortunately no contribution of BAT could be found.12,13 In the last decade, the evolvement of new imaging techniques made it possible to determine the functionality of human BAT. The development of positron-emissiontomography-and-computed-tomography (PET-CT), where the radioactive glucose tracer 2-deoxy-2-18 F-fluoro-D-glucose (FDG) shows cellular glucose uptake, gives the opportunity to depict metabolically active tissues. FDG-PET-CT studies are clinically widely used in oncological imaging. Interestingly, it was frequently observed that FDG uptake occurred in adipose tissue (Figure 2), ${ }_{1}^{14}$ located in anatomical areas that were described in anatomical studies earlier to contain BAT. ${ }^{3}$ This FDG-PET-CT-positive adipose tissue was mostly observed in patients feeling cold and repeated scanning under warm conditions prevented the uptake in those areas (Figure 3). ${ }^{15,16}$ This raised scientific interest from autopsies. ${ }^{17}$ Moreover, there was a significant negative correlation between the amount of active BAT and the

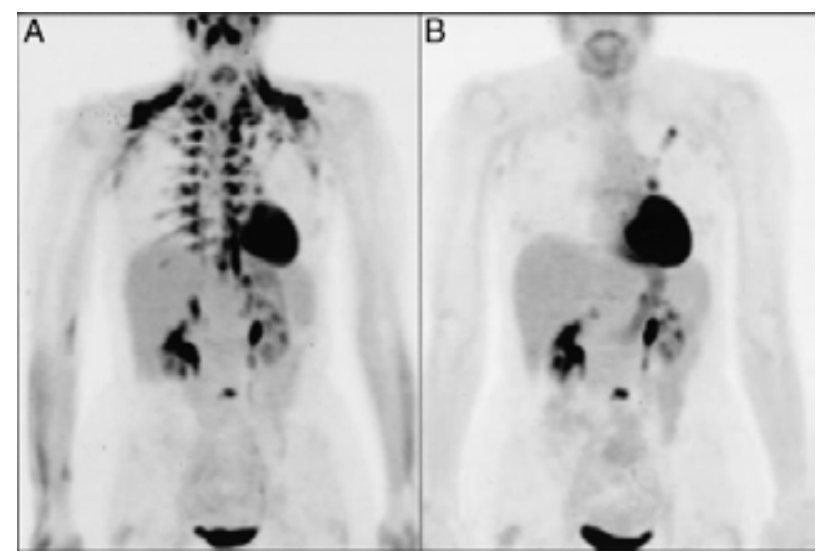

Figure 2. FDG-PET-CT-image of a female patient with non-small-cell lung cancer (NSCLC) showing increased FDG-uptake in periclavicular and paravertebral adipose tissue during uncontrolled conditions (A) and absence of FDG-uptake in adipose tissue during warm conditions (B; room temperature $22-25^{\circ}$ Celsius). Image obtained from Christensen et al. ${ }^{15}$ 
percentage of body fat and BMI, i.e. a low BAT activity in obesity (Figure 4). ${ }^{17}$ and a prospective study from our research group in young, healthy subjects under controlled mild cold exposure showed active BAT in the majority of the study group (96\%) in anatomical regions similar to descriptive observations. Adipose tissue biopsies taken from the regions that showed FDG-uptake on PET-CT during cold exposure confirmed the histological presence of brown adipocytes (Figure 5)..$^{17,18}$ Retrospective analyses of cohorts of clinically performed FDG-PET-CT studies (mostly performed in oncological settings) throughout development in childhood and adolescence showed that BAT peeks up to observed amounts of $\sim 1000 \mathrm{~g}{ }^{19}$

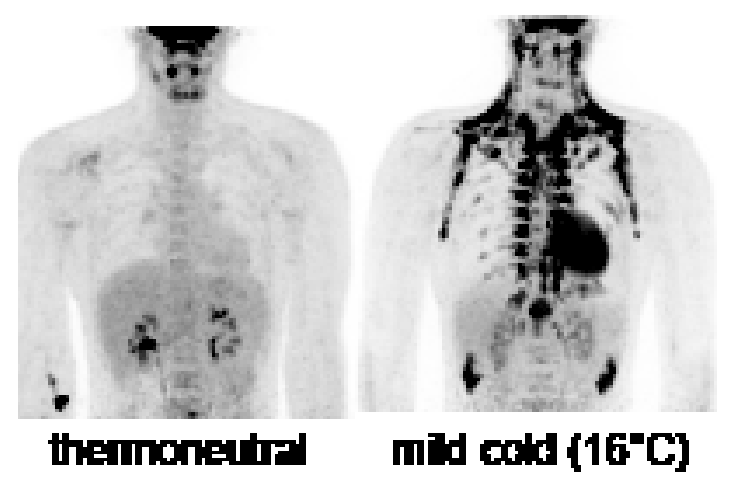

Figure 3. PET-CT-image of a healthy, young male subject under thermoneutral conditions (thermoneutral) and after mild cold exposure (mild cold). After mild cold exposure, the black areas indicate active uptake of FDG in supraclavicular and paravertebral adipose tissue. Images obtained from van Marken Lichtenbelt et al., NEJM, 2009..$^{17}$

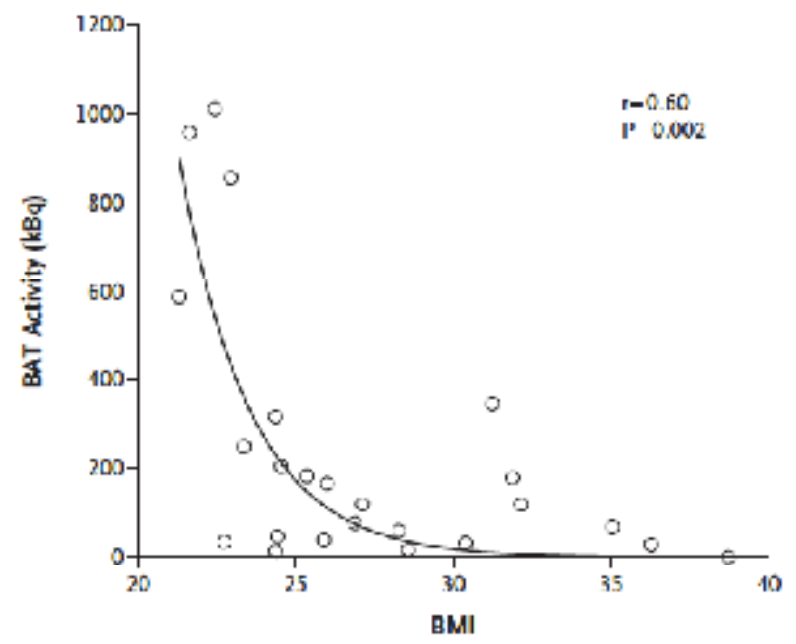

Figure 4. Relation between Body Mass Index (BMI) and Brown Adipose Tissue (BAT) activity. Image ontained from van Marken Lichtenbelt et al, NEJM, 2009. ${ }^{17}$ 


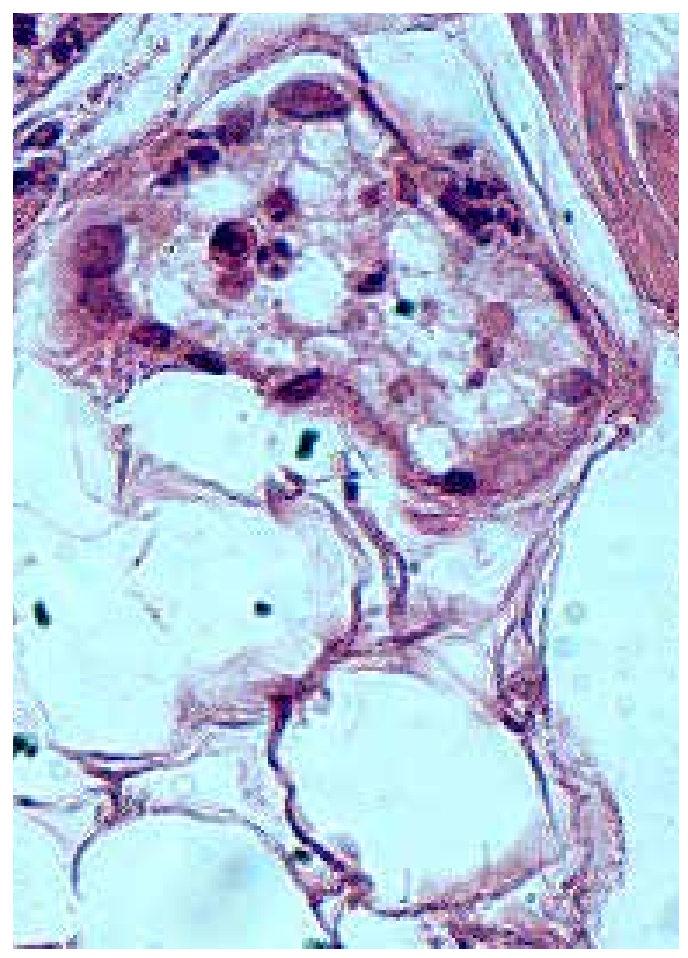

Figure 5. Formalin-fixed histological slide of supraclavicular human brown adipose tissue. The multivacuolar aspect and dark-stained mitochondria are evident in the brown adipocyte (Vijgen et al., unpublished work).

However, prospective, controlled studies in healthy adolescents using metabolic imaging techniques show average volumes of BAT of $\sim 100 \mathrm{~g}$ or $\sim 100 \mathrm{~cm}^{3}$ (range $\left.0-296 \mathrm{~cm}^{3}\right) .{ }^{17}$ Both the amount of BAT and the number of anatomical sites show that BAT decreases with age, resulting in a decline in BAT presence being restricted to the supraclavicular/neck area or even a total absence in the last decades of life. ${ }^{3,17,20}$ The mechanism underlying this change in volume and distribution of BAT through life is unknown.Interestingly, recent cell studies suggest that the amount and function of brown adipocytes can change. During the development of adipocytes several factors have been identified that can influence the final differentiation into white and brown phenotypes. ${ }^{21,22}$ Recent reports show that stimulation of WAT depots triggers the growth of a subset of thermogenically active BRITE (brown-in-white) or 'beige' cells. ${ }^{23-25}$ This suggests that within human WAT a subset of preadipocytes have a certain 'plasticity', which allows differentiation to thermogenic cells depending on environmental conditions or specific stimuli. Interestingly, recently biopsies from the supraclavicular adipose tissue area known to contain BAT in man have shown the presence of beige adipocytes and preadipocytes that could become thermogenically active in vitro. ${ }^{26,27}$ This 'plasticity' of BAT could 
explain the previously observed high amounts of BAT in Finnish outdoor workers, who were exposed for longer periods of cold compared to their colleague indoor workers. ${ }^{28}$ Therefore, possibly BAT in adult man could increase when stimulated to a similar extent as long-term cold exposure. Theoretically, BAT grafts developed ex-vivo could be implanted in subjects with low or absent BAT. However, this is not possible yet and at this moment most studies focus on determining the physiological and pathophysiological importance of BAT in man. In summary, BAT could have a significant role in human physiology and possibly be important in the regulation of the energy balance and weight maintenance, similar to the situation in small mammals. Therefore, this raised renewed interest in BAT in adult humans and its metabolic role in relation to obesity.

\section{Role of brown adipose tissue in obesity}

Obesity and its comorbidities were already stated as diseases by Hippocrates in his De Priscina Medicina (400 BC): "corpulence is not only a disease itself, but the harbinger of other diseases". 29,30 In the past under more natural living conditions, the excess amount of adipose tissue in obesity served as an energy source during periods of food scarcity. In modern times food is always available and more than $10 \%$ of the world's adult population is obese, making obesity one of the biggest health threats in the Western world. ${ }^{31,32}$ Obesity is strongly associated with other diseases, such as type II diabetes, cardiovascular disease, dyslipidemia and cancer. ${ }^{33}$ The rapidly increasing incidence of obesity and its associated diseases demand for both a preventive and curative strategy. Most obesity therapies aim at restoring the disturbed energy balance by either decreasing energy intake or increasing energy expenditure. Caloric restriction may be successful on short term, ${ }^{34}$ but unfortunately it is extremely difficult to maintain weight loss, as shown by long-term follow-up studies. ${ }^{35}$ One of the first promising drugs targeting obesity was 2,4-dinitrophenol (DNP), developed in the 1930s. DNP caused rapid weight loss by increasing energy expenditure through uncoupling of mitochondrial oxidative phosphorylation. ${ }^{36}$ However, severe adverse effects such as hyperthermia made this drug unsuitable for large-scale use. During the last decades, several drugs were released that aimed at decreasing energy intake. ${ }^{37}$ These substances targeted the central nervous system (sibutramine, rimonabant, phentermine, diethylpropion), cellular (rosiglitazone) and digestive components (orlistat) of obesity and initially showed significant long-term weight losses of 2.9-4.7 kg up to 5-10 kg after 2 years. ${ }^{34,37,38}$ However, increased cardiovascular risks (rosiglitazone), mood disorders (rimonabant) and abdominal 
complaints (orlistat) made that these drugs are currently not recommended anymore. ${ }^{37}$ Therefore, more rigorous treatments are sometimes inescapable, for example in morbid obesity. In these patients invasive bariatric surgery is an effective long-term weight loss therapy. ${ }^{39,40}$ In search for less invasive treatments, the quest for the 'holy grail' in obesity treatment continues and in the past years BAT has gained increasing scientific interest as a possible therapeutic target tissue for obesity in man.

In man, most studies using imaging techniques first expose study subjects to mild cold to induce BAT activity. The mild cold exposure sensitizes skin neurons and is then believed to stimulate an increase in sympathetic nervous system (SNS) activity. This increased SNS activity stimulates synapse norepinephrine (NE) release which induces $ß$-adrenergically-mediated BAT activity. ${ }^{5}$ Interestingly, although $ß$-receptors are present in human $\mathrm{BAT}^{18}$ the exact $\beta$-adrenergic stimulus is not known yet. Recent imaging studies targeting $ß$-adrenergic BAT stimulation with isoprenaline and ephedrine $(1.0 \mathrm{mg} / \mathrm{kg}$ ) were unsuccessful and only high doses of ephedrine $(2.5 \mathrm{mg} / \mathrm{kg})$ were able to induce some BAT activity in lean but not obese humans. ${ }^{41-}$ 43 Therefore, a more detailed, cellular investigation of BAT is necessary to identify the molecular mechanisms that eventually induce UCP1-mediated mitochondrial uncoupling in the brown adipocyte to target BAT activation.

In summary, most therapeutic strategies for obesity aiming at a long-term reduction of energy intake are unsuccessful and to date no treatment has been able to sustainably increase energy expenditure. Possibly, BAT can be of great value in future obesity treatment. This thesis deals with the physiology of BAT in obesity and therapeutic options to induce BAT activity in man are explored.

\section{Outline of the thesis}

This thesis describes studies on the physiology and pathophysiology of BAT in man, in relation to the treatment of obesity. In Chapter 2, the clinical relevance of BAT in man is discussed. In Chapter 3, we observed BAT activity in morbidly obese subjects in comparison to previously studied lean subjects. Next, the effect of major weight loss by bariatric surgery in morbidly obese subjects on presence and activity of BAT was studied (Chapter 4). Since recent studies have shown that heat production in BAT and skeletal muscle could be closely related, we studied the effect of weight loss on skeletal muscle mitochondrial function (Chapter 5). Chapter 6 describes a study on the cellular physiology and the metabolic capacity of human brown and white adipose tissue. Chapter 7 describes a study on the effect of central nervous 
system stimulation on BAT activity in patients with refractory epilepsy who are treated with vagus nerve stimulation. Finally, in Chapter 8 , the results obtained in this thesis are discussed and implications and applications for further research are presented. 


\section{References}

1. Gesner C, Rondelet G, Belon P, Carronus J, Wolf C. Historiae Animalium; De Quadrupedibus viuiparis; Liber Primus. 1620;Editio secunda:744.

2. Cannon B, Nedergaard J. Developmental biology: Neither fat nor flesh. Nature 2008;454:947-8.

3. Heaton JM. The distribution of brown adipose tissue in the human. J Anat 1972;112:35-9.

4. Fruhbeck G, Becerril S, Sainz N, Garrastachu P, Garcia-Velloso MJ. BAT: a new target for human obesity? Trends Pharmacol Sci 2009;30:387-96.

5. Cannon B, Nedergaard J. Brown adipose tissue: function and physiological significance. Physiol Rev 2004;84:277-359.

6. van Marken Lichtenbelt WD, Schrauwen P. Implications of non-shivering thermogenesis for energy balance regulation in humans. Am J Physiol Regul Integr Comp Physiol 2011.

7. Feldmann HM, Golozoubova V, Cannon B, Nedergaard J. UCP1 ablation induces obesity and abolishes diet-induced thermogenesis in mice exempt from thermal stress by living at thermoneutrality. Cell Metab 2009;9:203-9.

8. Rothwell NJ, Stock MJ. A role for brown adipose tissue in diet-induced thermogenesis. Nature 1979;281:31-5.

9. Kozak LP. Brown fat and the myth of diet-induced thermogenesis. Cell Metab 2010;11:263-7.

10. Nicholls DG, Bernson VS, Heaton GM. The identification of the component in the inner membrane of brown adipose tissue mitochondria responsible for regulating energy dissipation. Experientia Suppl 1978;32:89-93.

11. Heaton GM, Wagenvoord RJ, Kemp A, Jr., Nicholls DG. Brown-adiposetissue mitochondria: photoaffinity labelling of the regulatory site of energy dissipation. Eur J Biochem 1978;82:515-21.

12. Astrup A, Bulow J, Christensen NJ, Madsen J. Ephedrine-induced thermogenesis in man: no role for interscapular brown adipose tissue. Clin Sci (Lond) 1984;66:179-86.

13. Astrup A, Bulow J, Madsen J, Christensen NJ. Contribution of BAT and skeletal muscle to thermogenesis induced by ephedrine in man. Am J Physiol 1985;248:E507-15.

14. Yeung HW, Grewal RK, Gonen M, Schoder H, Larson SM. Patterns of (18) F-FDG upwwtake in adipose tissue and muscle: a potential source of false-positives for PET. J Nucl Med 2003;44:1789-96. 
15. Christensen CR, Clark PB, Morton KA. Reversal of hypermetabolic brown adipose tissue in F-18 FDG PET imaging. Clin Nucl Med 2006;31:193-6.

16. Nedergaard J, Bengtsson T, Cannon B. Unexpected evidence for active brown adipose tissue in adult humans. Am J Physiol Endocrinol Metab 2007;293:E444-52.

17. van Marken Lichtenbelt WD, Vanhommerig JW, Smulders NM, et al. Cold-activated brown adipose tissue in healthy men. N Engl J Med 2009;360:15008.

18. Virtanen KA, Lidell ME, Orava J, et al. Functional brown adipose tissue in healthy adults. N Engl J Med 2009;360:1518-25.

19. Gilsanz V, Smith ML, Goodarzian F, Kim M, Wren TA, Hu HH. Changes in Brown Adipose Tissue in Boys and Girls during Childhood and Puberty. J Pediatr 2011.

20. Yoneshiro T, Aita S, Matsushita M, et al. Brown Adipose Tissue, WholeBody Energy Expenditure, and Thermogenesis in Healthy Adult Men. Obesity (Silver Spring) 2011;19:13-6.

21. Tseng $\mathrm{YH}$, Kokkotou E, Schulz TJ, et al. New role of bone morphogenetic protein 7 in brown adipogenesis and energy expenditure. Nature 2008;454:10004.

22. Seale P, Bjork B, Yang W, et al. PRDM16 controls a brown fat/skeletal muscle switch. Nature 2008;454:961-7.

23. Petrovic N, Walden TB, Shabalina IG, Timmons JA, Cannon B, Nedergaard J. Chronic peroxisome proliferator-activated receptor gamma (PPARgamma) activation of epididymally derived white adipocyte cultures reveals a population of thermogenically competent, UCP1-containing adipocytes molecularly distinct from classic brown adipocytes. J Biol Chem 2009;285:7153-64.

24. Wu J, Bostrom P, Sparks LM, et al. Beige adipocytes are a distinct type of thermogenic fat cell in mouse and human. Cell 2012;150:366-76.

25. Bostrom P, Wu J, Jedrychowski MP, et al. A PGC1-alpha-dependent myokine that drives brown-fat-like development of white fat and thermogenesis. Nature 2012;481:463-8.

26. Wu ea. Beige Adipocytes Are a Distinct Type of Thermogenic Fat Cell in Mouse and Human. Cell 2012.

27. Lee P, Swarbrick MM, Ting Zhao J, Ho KK. Inducible Brown Adipogenesis of Supraclavicular Fat in Adult Humans. Endocrinology 2011.

28. Huttunen P, Hirvonen J, Kinnula V. The occurrence of brown adipose tissue in outdoor workers. Eur J Appl Physiol Occup Physiol 1981;46:339-45. 
29. Haslam DW, James WP. Obesity. Lancet 2005;366:1197-209.

30. Haslam D. Obesity: a medical history. Obes Rev 2007;8 Suppl 1:31-6.

31. WHO. Action plan for the global strategy for the prevention and control of noncommunicable diseases. 2009.

32. WHO Fact sheet No 311 - Obesity and overweight. 2011. (Accessed at www.who.int/mediacentre/factsheets/fs311/en/index.html )

33. Obesity: preventing and managing the global epidemic. Report of a WHO consultation. World Health Organ Tech Rep Ser 2000;894:i-xii, 1-253.

34. Eckel RH. Clinical practice. Nonsurgical management of obesity in adults. N Engl J Med 2008;358:1941-50.

35. Simpson SA, Shaw C, McNamara R. What is the most effective way to maintain weight loss in adults? BMJ 2011;343:d8042.

36. Grundlingh J, Dargan PI, El-Zanfaly M, Wood DM. 2,4-dinitrophenol (DNP): a weight loss agent with significant acute toxicity and risk of death. J Med Toxicol 2011;7:205-12.

37. Padwal RS, Majumdar SR. Drug treatments for obesity: orlistat, sibutramine, and rimonabant. Lancet 2007;369:71-7.

38. Douketis JD, Macie C, Thabane L, Williamson DF. Systematic review of long-term weight loss studies in obese adults: clinical significance and applicability to clinical practice. Int J Obes (Lond) 2005;29:1153-67.

39. Sjostrom L, Lindroos AK, Peltonen M. Lifestyle, diabetes, and cardiovascular risk factors 10 years after bariatric surgery. N Engl J Med 2004;351:2683-93.

40. Sjostrom L, Narbro K, Sjostrom CD, et al. Effects of bariatric surgery on mortality in Swedish obese subjects. N Engl J Med 2007;357:741-52.

41. Vosselman MJ, van der Lans AA, Brans B, et al. Systemic beta-Adrenergic Stimulation of Thermogenesis Is Not Accompanied by Brown Adipose Tissue Activity in Humans. Diabetes 2012.

42. Cypess AM, Chen YC, Sze C, et al. Cold but not sympathomimetics activates human brown adipose tissue in vivo. Proc Natl Acad Sci U S A 2012;109:10001-5. 43. Carey $A L$, Formosa MF, Van Every $B$, et al. Ephedrine activates brown adipose tissue in lean but not obese humans. Diabetologia 2012. 


$$
\theta_{-1}
$$




\section{Chapter 2}

\section{Brown adipose tissue; clinical impact of a re- discovered thermogenic organ}




\begin{abstract}
Brown adipose tissue (BAT) is physiologically present and active in adult humans. The stimulation of BAT in man can potentially increase total daily energy expenditure and is seen as a possible target to treat obesity. Altered BAT activity is also related to other diseases and therefore the therapeutic potential of BAT could reach beyond obesity. This is supported by both in vitro and in vivo reports from animal studies, describing the possible role of BAT in both physiology and pathophysiology. In addition, since the discovery of functional BAT several clinically relevant studies have been conducted in adult man. Clinical observations report BAT activity under multiple conditions, suggesting BAT could be important in the onset and/or treatment of diseases related to the metabolic syndrome, thyroid disorders, cancer and immune system dysfunction. This review highlights those diseases or syndromes in which BAT may play a role in relation to prevention, diagnosis or therapy, by translating basic research into clinical relevance.
\end{abstract}




\section{Introduction}

Recently, active brown adipose tissue (BAT) was discovered in healthy adults. ${ }^{1-3}$ In rodents, BAT is known for of its high heat production capacity that can significantly increase total energy expenditure. ${ }^{4}$ Since the discovery of functional BAT in man, increasing energy expenditure by the stimulation of BAT is seen as a new anti-obesity target. ${ }^{5,6}$ Interestingly, in man BAT activity is low or absent in obese subjects. Possibly, increasing energy expenditure by stimulation of BAT could be important in weight maintenance or could create a negative energy balance leading to weight loss.

Studies in both man and animals also show active BAT under several clinical conditions. These studies suggest involvement of BAT in conditions that are associated with the metabolic syndrome (obesity, type 2 diabetes, dyslipidemia, fatty liver disease), endocrine disorders (pheochromocytoma, thyroid gland dysfunction), neoplasms (cancer cachexia, hibernoma), general inflammation and the immune response. Here, we will discuss the relevance of BAT in relation to these conditions, and its possible therapeutic impact.

\section{Physiology of BAT}

Classically, BAT is associated with cold exposure. To prevent hypothermia, increasing insulation (clothing) and change of environment are effective behavioral strategies. However, if this is not possible or insufficient, generating body heat is necessary to prevent hypothermia. Shivering in skeletal muscle is very effective to increase heat production, but this cannot be sustained for longer periods of time.? Interestingly, when a subject is exposed to cold, heat production already increases prior to the onset of shivering. ${ }^{8}$ This process is defined as nonshivering thermogenesis (NST). In rodents BAT is the main site for NST. ${ }^{4}$ In man, there are indications that both BAT and skeletal muscle are involved. ${ }^{9}$

\section{Mitochondrial uncoupling induces thermogenesis in BAT}

NST in the brown adipocyte is facilitated by 'uncoupling' of the mitochondrial respiration from adenosine-tri-phosphate (ATP) production. The mitochondrial respiration chain generates a proton gradient across the inner mitochondrial membrane to produce ATP. However, during BAT stimulation the cross-membrane proton gradient is used to produce heat instead of ATP. An essential protein for this process, that also typifies BAT, is the uncoupling protein 1 (UCP1, Figure 1). ${ }^{4}$ In order 


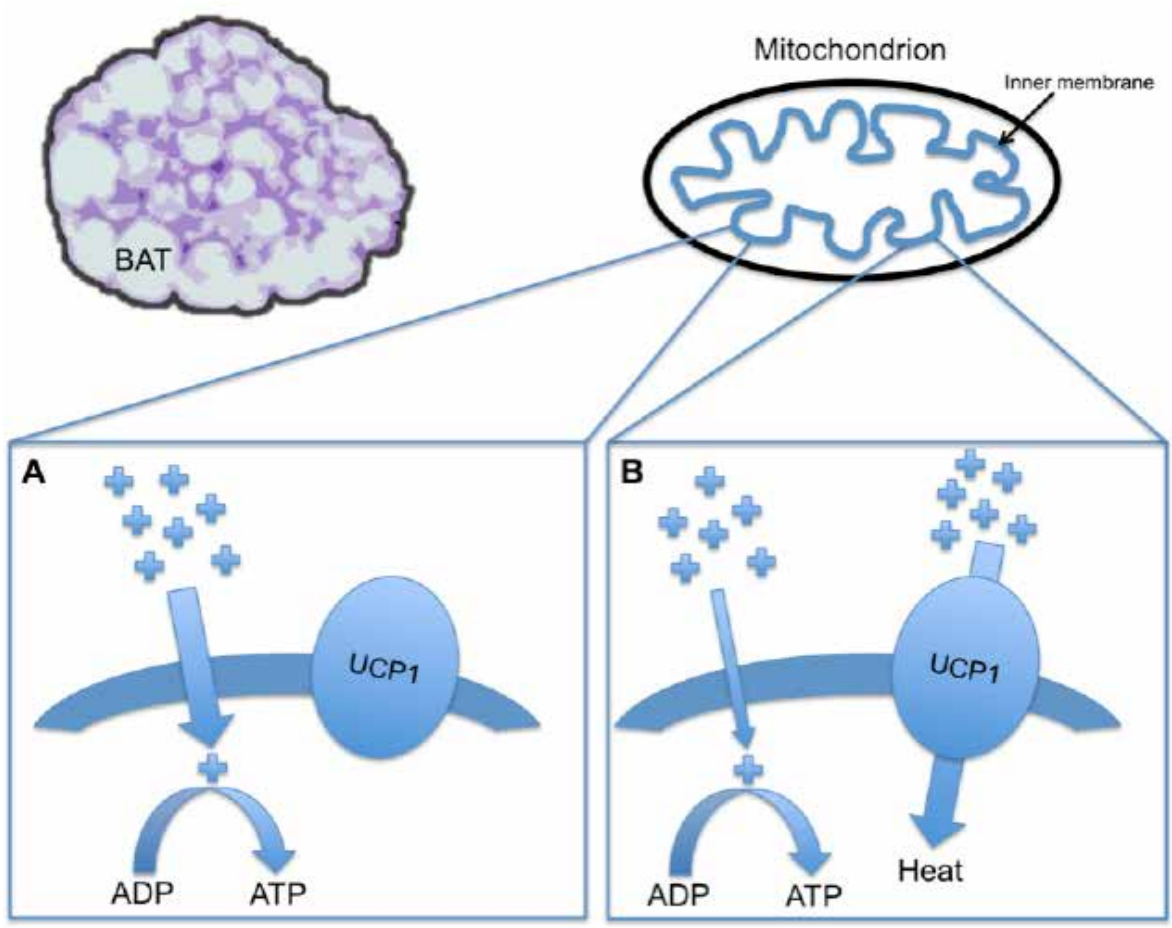

Figure 1. Uncoupling protein 1 (UCP1) mediates mitochondrial uncoupling in brown adipose tissue (BAT). Schematic overview of BAT (top left), BAT mitochondrion (top right) and inner mitochondrial membrane (A and B). The proton gradient across the inner mitochondrial membrane can be used to generate ATP (coupled respiration, depicted in A). When BAT is stimulated to become active, UCP1 facilitates uncoupling of ATP-generation to induce thermogenesis (uncoupled respiration, depicted in B).

to facilitate uncoupling by UCP1 in the brown adipocyte, free fatty acids (FFAs) are necessary. ${ }^{4}$ The exact contribution of FFAs to UCP1 activation is still unknown, but most likely they facilitate a membrane entrance for protons to generate thermogenesis. ${ }^{4}$ Besides FFAs, BAT also uses glucose during thermogenesis. ${ }^{4}$ The purpose of glucose-uptake in active BAT is largely unknown. Possibly, glucose is necessary to maintain the citric cycle, ATP production and the proton gradient ${ }^{4}$. Alternatively, glucose is converted to FFAs. ${ }^{4}$ The function of glucose uptake in BAT would then be to increase the amount of FFAs, necessary for mitochondrial uncoupling.

\section{Involvement of sympathetic nervous system in BAT activation}

The sympathetic nervous system (SNS) stimulates BAT to produce heat during NST. Sympathetic innervation is essential for BAT to become active, as disruption of the sympathetic fibers to BAT completely ceased BAT activity. ${ }^{10}$ In man, most 
likely responses from warm- and cold sensitive neurons regulate the activation of thermogenesis. ${ }^{11}$ In the hypothalamus and medulla input from these neurons is mediated into sympathetic stimuli that control BAT thermogenesis by the release of norepinephrine on the adrenergic receptors of BAT cells. ${ }^{11}$ In rodents, BAT activity is associated with both adrenergic $\alpha$ - and $\beta$-receptor subtypes. Although the $\alpha_{1 A}$-receptor is abundant in BAT, stimulation could only account for maximally $10 \%$ of total BAT thermogenesis in rodents. ${ }^{4}$ The $\alpha_{2}$-receptor is even suggested to inhibit thermogenesis. The $\beta_{3}$-receptor seems to be the most significant mediator of thermogenesis, as suggested from studies that have compared BAT stimulation with $\beta_{3}$-agonists versus norepinephrine. ${ }^{4}$ Most likely $\beta_{3}$-activation, and to a much lower extent $\alpha_{1 A}$-activation, mutually mediate norepinephrine-induced thermogenesis in mammals. Although present in BAT, the $B_{1}$-receptor is only suggested to influence BAT activity when $\beta_{3}$-receptor signaling is dysfunctional (e.g. $\beta_{3}$-receptor-ablation). ${ }^{4}$

\section{Thyroid hormone induces BAT activation}

In addition to sympathetic stimulation of BAT, thyroid hormone plays a significant role in BAT thermogenesis. The thyroid gland mainly secretes the inactive pro-hormone thyroxine (T4), which needs to be deiodinised (lose an iodine atom) by deiodinases to triiodothyronine (T3) to become active. In rodents, T3 can stimulate BAT. ${ }^{12}$ After conversion of T4 to T3 by the BAT-specific type-II-iodothyronine-5deiodinase (D2), thyroid hormone enhances BAT thermogenesis. ${ }^{12,13}$ In addition, thyroid hormone suppletion in the brain increased BAT activity and induced weight loss in rodents. ${ }^{13}$ This activation of BAT could then be reversed by the $₫ 3$-adrenergic receptor antagonist SR59230A, indicating this central thyroid activation was mediated via the SNS. ${ }^{13}$ These results indicate interplay at the central nervous system level between the endocrine (thyroid axis) and sympathetic regulation of BAT.

\section{Presence of BAT in man}

In humans, BAT is present in significant amounts during childhood. Compared to adults, newborns have several disadvantages regarding their maintenance of body temperature. The higher body surface-to-volume ratio may cause a more rapid heat loss. Due to low amount of muscle, the ability to induce thermogenesis by shivering is limited. Therefore NST is important in newborns. ${ }^{4}$ During infancy growth changes the relative surface area and the amount of BAT gradually decreases. In adulthood BAT has been reported in autopsies, ${ }^{14}$ but it was always believed to be dysfunctional. ${ }^{15}$ 
Strikingly, BAT has recently shown to be present in significant amounts in adults and becomes functionally active upon cold exposure. ${ }^{1,2} 2$-deoxy-2-18 F-fluoro-Dglucose $\left({ }^{18} \mathrm{~F}-\mathrm{FDG}\right)$, a radioactive labeled glucose tracer, is used in nuclear imaging (positron-emission-tomography-and-computed-tomography (PET-CT)) to depict glucose uptake for tumor diagnosis. Frequently non-malignant uptake in adipose tissue was seen, which later appeared to be related to mild cold conditions during the tracer administration. Prospective, controlled mild cold exposure consistently showed this activity in supraclavicular, cervical and paravertebral adipose tissue regions. Tissue biopsies from PET-active supraclavicular adipose tissue regions confirmed BAT presence. ${ }^{1,2}$

Retrospective ${ }^{18} \mathrm{~F}$-FDG PET-CT series in infants show high amounts of BAT that even increase during adolescence. ${ }^{16}$ In adolescence, muscle mass also still increases under influence of metabolic and hormonal factors. ${ }^{16}$ Seale et al. showed that skeletal muscle and BAT share a similar developmental origin ${ }^{17}$ and therefore it is suggested the factors that induce muscle growth during adolescence could similarly influence BAT. ${ }^{16}$ After the volume of BAT peeks up to amounts of $1200 \mathrm{~cm}^{3}$ in the second decade of life, ${ }^{16}{ }^{18} \mathrm{~F}$-FDG PET-CT studies ${ }^{3}$ and histological analyses ${ }^{18}$ indicate the amount of BAT decreases with age. BAT expresses sex hormone receptors and the age-related involution of BAT could be influenced by the lowering levels of sex hormones. ${ }^{19}$ If low BAT is related to disease, the age-related BAT loss could be important in the onset of the metabolic syndrome and other pathologic conditions.

\section{BAT in disease}

\section{Metabolic syndrome}

\section{Obesity}

In mice, absence of active BAT through ablation of UCP1 causes a weight gain of $>50 \%$ within 30 days after birth. ${ }^{20}$ In man, dedicated studies show a strong negative correlation with BAT activity and body fat percentage and body mass index.1,3,21 Retrospective analyses of patient ${ }^{18} \mathrm{~F}-\mathrm{FDG}$ PET-CT-scans reveal comparable relations. ${ }^{22-25}$ Possibly, due to the insulation by subcutaneous adipose tissue in obese subjects there is less need to activate BAT in daily living situations. Subjects without BAT activity show significantly lower NST, ${ }^{21,26}$ suggesting BAT is involved in the human energy balance. A classic overfeeding study in man showed 
large differences in weight gain on a high-calorie diet. ${ }^{27}$ If low or absent BAT activity would be responsible for the differences in weight gain after overfeeding, increasing BAT activity could possibly prevent or treat obesity. An explanation for the individual variation in BAT could be associated with polymorphisms in the UCP1-gene. Resting energy expenditure, sympathetic nervous system activity and the thermal effect of a meal show a relation with the UCP1 G/G genotype. ${ }^{28}$ Moreover, if the UCP1 G/G genotype is accompanied by a Trp64Arg polymorphism of the $\beta_{3}$-receptor gene this is associated with a lower basal metabolic rate, lower sympathetic nervous system activity and a higher amount of visceral adipose tissue. ${ }^{29,30}$ However, there are no studies that have directly related BAT activity and UCP1-polymorphisms yet.

In summary, active BAT is low or absent in obesity. Body composition (body fat percentage) and environmental factors (lifestyle) may cause variations in amount and activity of BAT. Increasing BAT activity could be a new target to both prevent and treat overweight.

\section{Type 2 diabetes}

The significant uptake of glucose in active BAT is shown by ${ }^{18} \mathrm{~F}-\mathrm{FDG}$ PET-CT. Stimulating glucose uptake in BAT can be beneficial in situations of impaired glucose clearance, as in type 2 diabetes. Indeed, retrospective cohort studies suggest an association with high blood glucose levels and absence of active BAT on ${ }^{18} \mathrm{~F}$-FDG PET-CT. ${ }^{24,31,32}$ Interesting is the case of a patient with a resected thyroid gland because of thyroid cancer and an extreme insulin resistance due to an insulin receptor mutation. ${ }^{33}$ The therapeutic treatment of this patient with high doses of thyroid hormone was accompanied with an increase of active BAT. The suppletion with thyroid hormone also resolved the patient's hyperglycaemia, hinting towards an association between BAT activity and glucose homeostasis.

To estimate the glucose clearing capacity of BAT in man, the uptake of glucose in cold-stimulated BAT can be used. Calculations made from dynamic ${ }^{18} \mathrm{~F}$-FDG PET-CT scans during cold exposure show a mean glucose clearance in BAT of $9.1 \pm 5.1 \mu \mathrm{mol} / 100 \mathrm{~g} /$ minute $(\mathrm{n}=27)^{34}$ to $22.67 \pm 18.28 \mu \mathrm{mol} / 100 \mathrm{~g} /$ minute $(\mathrm{n}=5)$ (unpublished results). To assess the possible contribution of cold stimulated BAT on whole body glucose clearance, glucose uptake in BAT $(0.7 \mathrm{mMol}$ glucose for $100 \mathrm{~g}$ 
BAT per hour, based on reports above) can be compared to the glucose infusion rate in a hyperinsulinemic-euglycemic clamp test $\left(166 \mathrm{mMol}\right.$ glucose per hour). ${ }^{35}$ In this comparison, BAT accounts for $0.4 \%$ of the body's maximal glucose uptake. In summary, active BAT takes up glucose and BAT in man can be important in glucose clearance. Therefore, BAT is a new potential target tissue for the treatment of type 2 diabetes.

\section{Dyslipidemia}

FFAs are essential for BAT thermogenesis (see Physiology of BAT). They are delivered to BAT by plasma circulating triglyceride-rich lipoproteins (TRLs) and cold-activated BAT in mice cleared a large amount of TRLs from the blood after a meal. ${ }^{36}$ On a cellular level, BAT-released lipoprotein lipase (LPL) converted the TRLs to free fatty acids (FFA), then used for thermogenesis. ${ }^{36}$ In conclusion, after BAT activation by cold exposure experimentally induced hyperlipidemia could be corrected. Although the condition of cold exposure was extreme $\left(4^{\circ} \mathrm{C}\right)$, the reported high clearance of plasma triglycerides ( $>50 \%$ ) provides hope for the treatment of dyslipidemia in man. In man, current treatment for dyslipidemia with statins generates a 40-60\% reduction in plasma low density lipoprotein (LDL) cholesterol, a direct lipolysis product of TRLs. ${ }^{37}$ Increasing BAT activity theoretically requests more lipolysis and could positively affect dyslipidemia.

\section{Hepatosteatosis}

Obesity, type 2 diabetes and dyslipidemia are all associated with an increased accumulation of fat in the liver, defined as hepatosteatosis (or non-alcoholic fatty liver disease (NAFLD)). Hepatosteatosis is associated with a decrease in liver functionality and a high risk of developing steatohepatitis and liver cirrhosis. ${ }^{38} \mathrm{~A}$ retrospective analysis of ${ }^{18} \mathrm{~F}$-FDG PET-CT-scans suggests an inverse relation between BAT activity and hepatosteatosis. ${ }^{39}$ Subjects without BAT activity had a significantly higher risk of hepatosteatosis and BAT activity was inversely correlated with the 
level of liver steatosis and body mass index (BMI). In addition to the association with energy balance, genetic polymorphisms of UCP1 are also associated with hepatosteatosis. ${ }^{40}$

In conclusion, hepatosteatosis is associated with low BAT activity and prospective studies are needed to define the role of low BAT in the development of hepatosteatosis.

\section{Thyroid disease}

Patients with thyrotoxicosis (high levels of plasma T4 and T3 due to a toxic goiter) have an increase in resting energy expenditure (REE) up to 30\% that returns to normal values after treatment with thiamazole. ${ }^{41}$ Upon T3-suppletion (resulting in T3 values twice as high compared to normal) in healthy subjects basal metabolic rate (BMR) increased. ${ }^{42}$ During hypothyroidism (low plasma levels of thyroid hormone) REE decreases $20-25 \% .{ }^{43}$ Even the induction of subclinical variations in thyroid function by suppletion of thyroid hormone already caused changes in REE of $5-10 \% .^{43}$ This indicates thyroid function is relevant for at least REE in man, but the contribution to thermogenesis and BAT has not been clearly defined yet. Support for thyroid-induced BAT activity in man is given by the above-mentioned case report of a patient with a resected thyroid gland because of thyroid cancer. ${ }^{33}$ Treatment with thyroid hormone was accompanied with active BAT on ${ }^{18} \mathrm{~F}-\mathrm{FDG}$ PET-CT, without cold exposure.

Similar to the influence of thyroid hormone on REE, variations in thyroid function within normal ranges could also impair or stimulate BAT activity. Therefore, dedicated studies on thyroid function and BAT in man are necessary.

\section{Pheochromocytoma}

The pheochromocytoma is a tumor of chromaffin-cells. In man, chromaffin cells are present in the medulla of the adrenal gland and secrete the catecholamines norepinephrine and epinephrine. During cold exposure, BAT is activated by sympathetic stimuli from the brain that release norepinephrine to stimulate the adrenergic receptors on BAT cells. ${ }^{11}$ Catecholamines released in the blood plasma induce adrenergic responses similar to activation of the sympathetic nervous system. In rodents, suppletion of norepinephrine is known to be able to increase the activity of BAT up to a fourfold. ${ }^{4}$ In patients with pheochromocytoma, retrospective ${ }^{18} \mathrm{~F}$-FDG PET-CT studies often show high BAT activity even under thermoneutral conditions. ${ }^{44,45}$ Interestingly, the high levels of circulating plasma catecholamines 
in pheochromocytoma were significantly related to the level of BAT activity. ${ }^{25}$ This suggests that the principle of catecholamine-activated BAT (by suppletion of norepinephrine into the circulation) as observed in rodents also applies to man.

\section{Cancer cachexia}

During cancer cachexia high BAT activity is reported. In autopsy studies in man high BAT presence in cachectic cancer patients was already described years ago. ${ }^{46}$ Active BAT is also often reported in ${ }^{18} \mathrm{~F}$-FDG PET-CT-imaging of oncologic patients. ${ }^{47}$ It is hypothesized that the high production of cytokines in cancer stimulates thermogenesis in BAT, both via the hypothalamus and directly on BAT cells. This is supported by studies in mice that show tumor implantation or stimulation with carcinogenic factors increases BAT activity. ${ }^{48}$

Experimental denervation of BAT and $\beta_{3}$-receptor antagonism reduces the effect of a cytokine-induced increase in energy expenditure. ${ }^{49}$ Therefore, the tumor-induced high BAT activity is held responsible for the increased thermogenesis that causes body mass loss up to anorectic levels in cancer cachexia.

\section{Hibernoma}

In addition to the high BAT activity in physiological depots in patients with malignant tumors, BAT can be present within a tumor itself. ${ }^{50}$ The rare hibernoma, a benign lipomatous tumor of brown adipocytes, was visible on ${ }^{18} \mathrm{~F}$-FDG PET-CT-imaging. ${ }^{23}$ Although the hibernoma shows activity on ${ }^{18} \mathrm{~F}-\mathrm{FDG}$ PET-CT, its activity apparently seems not high enough to significantly induce weight loss. ${ }^{23}$ In spite of the fact large amounts of BAT are present in the hibernoma, it is not activated in daily life.

\section{Immune response}

In rodents BAT thermogenesis increases during fever. ${ }^{51}$ In the inflammatory state BAT serves as the main heat-producing tissue in rodents. ${ }^{51}$ After the injection of lipopolysaccharide (LPS), an endotoxin present in bacteria, BAT is triggered to increase thermogenesis which causes fever. ${ }^{51}$ The increase in BAT activity also occurs in response to sympathetic stimuli from the hypothalamus, which itself is stimulated by pyrogenic cytokines. ${ }^{11}$ Besides the sympathetic stimulus from the brain, macrophages in the blood of mice are able to release catecholamines that activate BAT. ${ }^{52}$ Interestingly, during fever BAT starts producing its own endogenous pyrogens. ${ }^{51}$ BAT is reported to produce interleukin-6 (IL-6), a pro-inflammatory 
cytokine normally secreted by cells of the immune system, e.g. macrophages. IL- 6 is not only present during fever, but is also important during inflammation and infection and is seen in other immune reactions. ${ }^{53}$ In fever, IL- 6 initiates the pyrogenic mediator prostaglandine E2 (PGE2) which in the hypothalamus increases the temperature setpoint. ${ }^{11}$ In this perspective BAT thus could be capable of both controlling the level of fever and increasing heat production. This is interesting, because it makes BAT not only a tissue that responds when stimulated, but also a controller of thermogenesis itself. Cyclooxygenase 2 (COX-2), an important controller of PGE2 production, recruits BRITE (brown-in-white) cells in white adipose tissue (WAT) of mice. ${ }^{54}$ These cells are non-thermogenic preadipocytes present in WAT that differ from white adipocytes because they can become thermogenic to similar levels as BAT. ${ }^{55}$ Others factors for BRITE recruitment are a currently further explored treatment target (see Stimulation of BAT). Since IL-6, COX-2 and PGE2 are important in inflammation, increasing the amount of BAT or BRITE could be an interesting target to enhance the immune response during immunodeficiency.

Besides cold induced thermogenesis, BAT may be involved in immune competence of neonates. ${ }^{56}$ Sudden infant death syndrome (SIDS) is associated with a depletion of BAT, as observed afterwards during autopsy. ${ }^{56} \mathrm{It}$ is suggested this BAT depletion could follow from maternal conditions during pregnancy. Indeed, animal studies show that maternal cold exposure increased BAT activity in newborn lambs and lactation conditions were important for BAT development in mice. ${ }^{57,58}$

Results from autopsies, ${ }^{14}{ }^{18}$ F-FDG PET-CT-imaging studies ${ }^{3}$ and histological analyses ${ }^{18}$ indicate the amount of BAT decreases with age. If BAT is important in the immune response, a lower amount of BAT in the elderly could possibly account to more disease susceptibility.

In conclusion, BAT can be important for our immune system. However, more prospective studies on BAT activity or recruitment during fever or immune activation are needed.

\section{Stimulating BAT}

\section{Cold exposure}

Functional BAT in man was discovered by exposing subjects to mild cold., ${ }^{1,2}$ Under thermoneutral conditions, healthy subjects did not show active BAT. Indeed, active BAT is related to thermogenesis under mild cold exposure. ${ }^{21,26,59}$ During cold acclimatization (days to weeks) nonshivering thermogenesis (NST) increases. ${ }^{60}$ 
In mice, the increased NST after cold acclimatization originates from increased BAT activity. ${ }^{4}$ Cold acclimatization also causes recruitment of BAT in rodents and primates. ${ }^{4}$

In man, high volumes of BAT were observed in autopsy studies on Finnish outdoor workers, who were acclimatized to cold. ${ }^{61}$ Hence, cold acclimatization could be an interesting treatment modality to increase energy expenditure by activating NST in BAT. 7

\section{Insulin stimulation}

In mice, injection of insulin in the hypothalamus increases thermogenesis by activating BAT. ${ }^{62}$ In man, insulin infusion increased glucose uptake in BAT five-fold compared to baseline, from 0.9 to $4.7 \mu \mathrm{mol} / 100 \mathrm{~g} /$ minute. ${ }^{34}$ This insulin stimulated glucose uptake in BAT amounted to half of the cold stimulated BAT activity. In contrast to glucose uptake during cold exposure, insulin-stimulated glucose uptake in BAT is suggested to be more active under non-thermogenic conditions (no cold exposure). ${ }^{4}$ Under such conditions glucose would mainly be taken up in BAT to be converted into FFAs, that later can be used for mitochondrial uncoupling during BAT activation. ${ }^{4}$

In summary, BAT can be stimulated by insulin to take up glucose under thermoneutral conditions. Possibly, insulin-stimulated BAT could decrease blood glucose levels and serve as a therapeutic target for type 2 diabetes.

\section{Sympathetic stimulation}

Sympathetic ß-receptor agonists increase SNS activity, which could possibly stimulate BAT thermogenesis. Interestingly, cold-induced BAT activity in man can be fully blocked by the $ß$-antagonist propranolol. ${ }^{63}$ Propranolol is highly specific for $\beta_{1}$ - and $\beta_{2}$-receptors and rodent studies indicate BAT mainly contains $\beta_{3}$-receptors. ${ }^{4}$ This suggests BAT activity in man is mediated differently from rodents. The ß-agonist ephedrine was used to stimulate BAT and increase energy expenditure many years ago, but activation of BAT was not reported possibly due to the limited methods then available. ${ }^{15}$ Since ephedrine mainly stimulates $\beta_{1}$ - and $\beta_{2}$-receptors and propranolol shows blockage of these receptors inhibits BAT, ephedrine (or other ß-receptor agonists) could be effective in activating BAT in man after all. Controversially, propranolol suppletion did not reduce cold-induced NST in man. ${ }^{64}$ Since NST in man upon cold exposure is associated with BAT, this again supports 
the $B_{3}$-receptor specificity of BAT seen in rodents. These contrasting results could possibly become more clear after an intervention study with $B_{3}$-receptor specific

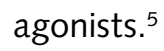

\section{BAT stimulation via the gut}

In rodents, the feeding of bile acids increases fat combustion in BAT. ${ }^{65}$ This signal is mediated via the $G$ protein-coupled bile acid-receptor TGR5 (TGR5), present on BAT. ${ }^{66}$ TGR5-agonists increase energy expenditure, decrease non-alcoholic fatty liver disease (NAFLD) and improve glucose tolerance in rodents. ${ }^{65}$ Interestingly, this process is dependent of the thyroid hormone converter D2 (for thyroid function and BAT, see BAT physiology). ${ }^{67}$ If D2 is disrupted in mice, bile acids do not increase BAT activity anymore, indicating the importance of thyroid-induced thermogenesis in bile acid-stimulated BAT activity. ${ }^{67}$

Gut hormones are involved in food intake regulation via the brain. ${ }^{68}$ Rodent studies indicate the central responses involved in ingestion are most likely located in the paraventricular hypothalamus, which is also known to induce the sympathetic nervous system (SNS) and thyroid axis. ${ }^{68}$ Increased SNS activity stimulates BAT activity, and therefore the response that mediates a decrease in food intake could stimulate energy expenditure in BAT. In man, this is illustrated by the observation that after suppletion of the appetite hormone oxyntomodulin energy expenditure was also stimulated. ${ }^{69}$

In summary, the suppletion of bile acids and gut hormones could induce an increase in BAT activity via the gut. ${ }^{70}$

\section{Transforming WAT to BAT}

All options described above consider the stimulation of BAT present at birth, often referred to as 'native' BAT depots. BAT in these depots was shown the share a developmental origin that is very similar to skeletal muscle. ${ }^{17}$ The presence or absence of the signaling molecule PRDM16 stimulates dermomyotomal precursors to end up as a skeletal muscle cell (absence of PRDM16) or as a brown adipocyte (PRDM16+). ${ }^{17}$ WAT seems to derive from a different developmental origin without the thermogenic capacity of BAT. ${ }^{11}$ However, within WAT depots a subpopulation of cells is indeed capable of developing into thermogenic 'beige' or BRITE (brownin-white) cells. ${ }^{55,71}$ These cells possess UCP1 and are thus capable of thermogenesis. Chaffee et al. already reported 'darker yellow adipose tissue' in rhesus monkeys after 12-24 months cold-acclimatization at $5^{\circ} \mathrm{C}$ in comparison to controls housed 
at $35^{\circ} \mathrm{C}{ }^{4}$. Several studies have now reported the development of BRITE cells upon diverse stimuli under cell culture conditions and in rodents. ${ }^{55,72,73}$ Here, three studies are of special clinical interest because of the use of human samples. ${ }^{73-75}$ Irisin, a novel hormone that induced 'browning' and UCP1-expression in subcutaneous adipose tissue from mice, was shown to increase in human plasma after 10 weeks of exercise. ${ }^{75}$ However, this study did not study the effects of irisin on human adipocytes and therefore further human studies are needed. Lee et al. show in vitro development of thermogenic adipocytes that derive from preadipocytes isolated from supraclavicular adipose tissue biopsies in both FDG-PET-CT-positive $(n=2)$ and FDG-PET-CT-negative $(n=4)$ subjects. ${ }^{74}$ The stimulants used in this study were both ß-agonists and proliferator-activated receptor gamma (PPAR-gamma), an agent present in several antidiabetic agents (rosiglitazone, pioglitazone). In another study, Schulz et al. treated preadipocytes isolated from human subcutaneous WAT with a mix of several agonists and showed the induction of UCP1 presence as a marker of BAT. ${ }^{73}$ Although no evidence exists of BRITE recruitment in vivo in man, the cell studies suggest that transformation of non-thermogenic to thermogenic fat cells is possible in man.

\section{Perspective}

Increasing evidence appears on the important role of BAT in adult physiology in both health and disease. Several recent studies illustrate possible therapeutic potentials of BAT (Table 1, Figure 2). This overview shows that in addition to nonshivering thermogenesis, BAT could be important in the metabolic syndrome (obesity, type 2 diabetes, dyslipidemia, hepatosteatosis), neoplasms (cancer cachexia, hibernoma) and the immune system. However, to define the clinical impact of BAT on disease more basic and clinical controlled studies are needed. Recent studies clearly show metabolic activity of BAT in adult humans, although there are indications of decreasing BAT activity with ageing. Therefore, the factors that account for this decrease should be targets for future investigations. Since it is considered unethical to use PET-CT-techniques to prospectively determine BAT activity in healthy infants and for repeated measurements in healthy adults, less invasive techniques need to be developed (like magnetic resonance imaging (MRI)). On the other hand it is feasible to perform clinical studies on BAT activity and recruitment during existing therapies. Thyroid hormone suppletion, insulin therapy, weight loss treatment and sympathetic stimulation can provide much insight in the dynamics of BAT and possible clinical targets. 
Table 1. Diseases associated with brown adipose tissue (BAT) activity and possible implications for therapy. Abbreviations: ${ }^{1}$ Brown adipose tissue

\begin{tabular}{|c|c|c|c|}
\hline Disease & Effect on $\mathrm{BAT}^{1}$ & Evidence & Potential implication \\
\hline Obesity & $\begin{array}{l}\text { Decrease of BAT } \\
\text { activity with increased } \\
\text { BMI and body fat } \%\end{array}$ & $\begin{array}{l}\text { Retrospective } \\
\text { (human) }{ }^{22-25,31,32} \\
\text { Prospective (human) }{ }^{1,3,21}\end{array}$ & $\begin{array}{l}\text { Increase BAT activity } \\
\text { to prevent and/ } \\
\text { or treat obesity }\end{array}$ \\
\hline Type 2 diabetes & $\begin{array}{l}\text { Association with high } \\
\text { blood glucose and } \\
\text { absence of BAT activity }\end{array}$ & $\begin{array}{l}\text { Retrospective } \\
\text { (human) }{ }^{24,31,32} \\
\text { Prospective evidence } \\
\text { of insulin-mediated } \\
\text { BAT activity }{ }^{34}\end{array}$ & $\begin{array}{l}\text { Increase BAT } \\
\text { activity to decrease } \\
\text { hyperglycemia }\end{array}$ \\
\hline Dyslipidemia & $\begin{array}{l}\text { BAT activation decreases } \\
\text { hyperlipidemia }\end{array}$ & Mice ${ }^{36}$ & $\begin{array}{l}\text { Increase BAT } \\
\text { activity to improve } \\
\text { hyperlipidemia }\end{array}$ \\
\hline Hepatosteatosis & $\begin{array}{l}\text { Association with } \\
\text { absence of BAT activity } \\
\text { and hepatosteatosis }\end{array}$ & Retrospective (human) ${ }^{39}$ & $\begin{array}{l}\text { Absence of BAT } \\
\text { activity could be } \\
\text { a risk to develop } \\
\text { hepatosteatosis }\end{array}$ \\
\hline Hyperthyroidism & $\begin{array}{l}\text { Increase in BAT activity } \\
\text { during hyperthyroidism }\end{array}$ & Rats $^{13}$ & \multirow{2}{*}{$\begin{array}{l}\text { Target thyroid } \\
\text { function to correct } \\
\text { impaired BAT activity }\end{array}$} \\
\hline Hypothyroidism & $\begin{array}{l}\text { Absence of BAT activity } \\
\text { during hypothyroidism }\end{array}$ & $\begin{array}{l}\text { Case-report after thyroid } \\
\text { resection (human) }{ }^{33}\end{array}$ & \\
\hline Pheochromocytoma & $\begin{array}{l}\text { Association with active } \\
\text { BAT and high plasma } \\
\text { catecholamine levels }\end{array}$ & $\begin{array}{l}\text { Pheochromocytoma } \\
\text { patients } 44,45\end{array}$ & $\begin{array}{l}\text { Stimulation of } \\
\text { sympathetic nervous } \\
\text { system to induce } \\
\text { BAT activity }\end{array}$ \\
\hline Cancer cachexia & $\begin{array}{l}\text { Active BAT during } \\
\text { cancer cachexia }\end{array}$ & $\begin{array}{l}\text { Tumor-bearing mice }{ }^{48} \\
\text { Autopsy (human) }{ }^{46} \\
\text { Cancer cachexia (human) }{ }^{47}\end{array}$ & $\begin{array}{l}\text { Cancer-induced } \\
\text { BAT activity } \\
\text { increases cachexia }\end{array}$ \\
\hline Hibernoma & $\begin{array}{l}\text { High amount of active } \\
\text { BAT in hibernoma }\end{array}$ & Human 23,50 & $\begin{array}{l}\text { Actived BAT in } \\
\text { hibernoma affects } \\
\text { energy balance }\end{array}$ \\
\hline Immune-activation & $\begin{array}{l}\text { Immune response } \\
\text { influences BAT activity }\end{array}$ & $\begin{array}{l}\text { Cell studies }{ }^{52} \\
\text { Mice }^{54}\end{array}$ & $\begin{array}{l}\text { BAT activity is } \\
\text { relevant in immune } \\
\text { activation }\end{array}$ \\
\hline
\end{tabular}




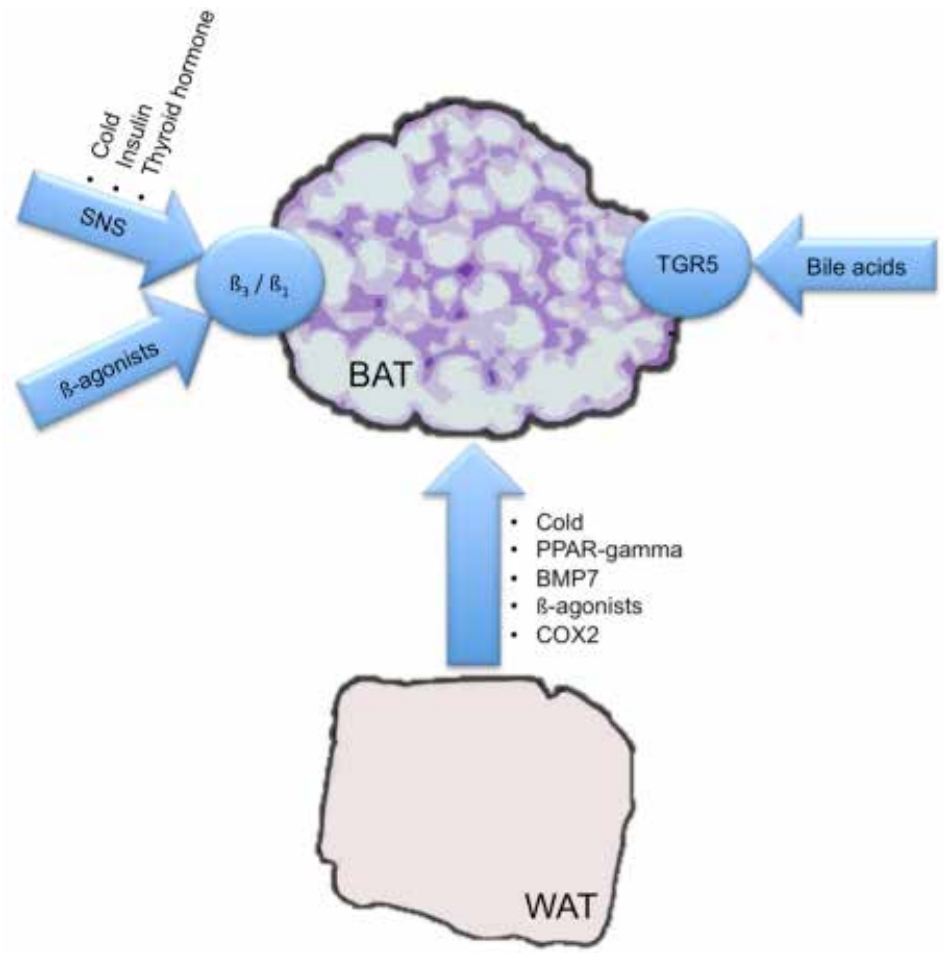

Figure 2. Potential therapeutic targets to stimulate BAT based on clinical observations. Schematic representation of brown adipose tissue (BAT) with beta-receptors $\left(\beta_{3}, \beta_{1}\right)$ and the bile-acid receptor TGR5. SNS indicates sympathetic nervous system, PPAR-gamma indicates peroxisome proliferator-activated receptor gamma, BMP7 indicates bone morphogenic protein 7, COX2 indicates cyclo-oxygenase-2, WAT indicates white adipose tissue. 


\section{References}

1. van Marken Lichtenbelt WD, Vanhommerig JW, Smulders NM, et al. Cold-activated brown adipose tissue in healthy men. N Engl J Med 2009;360:15008.

2. Virtanen KA, Lidell ME, Orava J, et al. Functional brown adipose tissue in healthy adults. N Engl J Med 2009;360:1518-25.

3. Saito $M$, Okamatsu-Ogura $Y$, Matsushita $M$, et al. High Incidence of Metabolically Active Brown Adipose Tissue in Healthy Adult Humans: Effects of Cold Exposure and Adiposity. Diabetes 2009;58:1526-31.

4. Cannon B, Nedergaard J. Brown adipose tissue: function and physiological significance. Physiol Rev 2004;84:277-359.

5. Tseng $\mathrm{YH}$, Cypess AM, Kahn CR. Cellular bioenergetics as a target for obesity therapy. Nat Rev Drug Discov 2010;9:465-82.

6. Nedergaard J, Bengtsson T, Cannon B. New powers of brown fat: fighting the metabolic syndrome. Cell Metab 2011;13:238-40.

7. van Marken Lichtenbelt WD, Schrauwen P. Implications of non-shivering thermogenesis for energy balance regulation in humans. Am J Physiol Regul Integr Comp Physiol 2011.

8. van Ooijen AM, van Marken Lichtenbelt WD, van Steenhoven AA, Westerterp KR. Cold-induced heat production preceding shivering. $\mathrm{Br} J \mathrm{Nutr}$ 2005;93:387-91.

9. Wijers SL, Schrauwen P, Saris WH, van Marken Lichtenbelt WD. Human skeletal muscle mitochondrial uncoupling is associated with cold induced adaptive thermogenesis. PLoS ONE 2008;3:e1777.

10. Lebron L, Chou AJ, Carrasquillo JA. Interesting image. Unilateral F-18 FDG uptake in the neck, in patients with sympathetic denervation. Clin Nucl Med 2010;35:899-901.

11. Morrison SF, Nakamura K. Central neural pathways for thermoregulation. Front Biosci 2011;16:74-104.

12. Bianco AC, Silva JE. Intracellular conversion of thyroxine to triiodothyronine is required for the optimal thermogenic function of brown adipose tissue. J Clin Invest 1987;79:295-300.

13. Lopez M, Varela L, Vazquez MJ, et al. Hypothalamic AMPK and fatty acid metabolism mediate thyroid regulation of energy balance. Nat Med 2010;16:10018.

14. Heaton JM. The distribution of brown adipose tissue in the human. J Anat 
1972;112:35-9.

15. Astrup A, Bulow J, Madsen J, Christensen NJ. Contribution of BAT and skeletal muscle to thermogenesis induced by ephedrine in man. Am J Physiol 1985;248:E507-15.

16. Gilsanz V, Chung SA, Jackson H, Dorey FJ, Hu HH. Functional brown adipose tissue is related to muscle volume in children and adolescents. J Pediatr 2011;158:722-6.

17. Seale P, Bjork B, Yang W, et al. PRDM16 controls a brown fat/skeletal muscle switch. Nature 2008;454:961-7.

18. Zingaretti MC, Crosta F, Vitali A, et al. The presence of UCP1 demonstrates that metabolically active adipose tissue in the neck of adult humans truly represents brown adipose tissue. FASEB J 2009;23:3113-20.

19. Nedergaard J, Cannon B. The changed metabolic world with human brown adipose tissue: therapeutic visions. Cell Metab 2010;11:268-72.

20. Feldmann HM, Golozoubova V, Cannon B, Nedergaard J. UCP1 ablation induces obesity and abolishes diet-induced thermogenesis in mice exempt from thermal stress by living at thermoneutrality. Cell Metab 2009;9:203-9.

21. Vijgen $\mathrm{GH}$, Bouvy ND, Teule GJ, Brans B, Schrauwen P, van Marken Lichtenbelt WD. Brown adipose tissue in morbidly obese subjects. Plos One 2011;6:e17247.

22. Pfannenberg C, Werner MK, Ripkens S, et al. Impact of age on the relationships of brown adipose tissue with sex and adiposity in humans. Diabetes 2010;59:1789-93.

23. Cypess AM, Lehman S, Williams G, et al. Identification and importance of brown adipose tissue in adult humans. N Engl J Med 2009;360:1509-17.

24. Ouellet V, Routhier-Labadie A, Bellemare W, et al. Outdoor temperature, age, sex, body mass index, and diabetic status determine the prevalence, mass, and glucose-uptake activity of 18F-FDG-detected BAT in humans. J Clin Endocrinol Metab 2011;96:192-9.

25. Wang $Q$, Zhang $M$, Ning $G$, et al. Brown adipose tissue in humans is activated by elevated plasma catecholamines levels and is inversely related to central obesity. Plos One 2011;6:e21006.

26. Yoneshiro T, Aita S, Matsushita M, et al. Brown Adipose Tissue, WholeBody Energy Expenditure, and Thermogenesis in Healthy Adult Men. Obesity (Silver Spring) 2011;19:13-6.

27. Bouchard C, Tremblay A, Despres JP, et al. The response to long-term overfeeding in identical twins. N Engl J Med 1990;322:1477-82. 
28. Nagai N, Sakane N, Kotani K, Hamada T, Tsuzaki K, Moritani T. Uncoupling protein 1 gene $-3826 \mathrm{~A} / \mathrm{G}$ polymorphism is associated with weight loss on a shortterm, controlled-energy diet in young women. Nutr Res 2011;31:255-61.

29. Valve R, Heikkinen S, Rissanen A, Laakso M, Uusitupa M. Synergistic effect of polymorphisms in uncoupling protein 1 and beta3-adrenergic receptor genes on basal metabolic rate in obese Finns. Diabetologia 1998;41:357-61.

30. Tsunekawa K, Yanagawa Y, Aoki T, et al. Association between accumulation of visceral fat and the combination of beta3 adrenergic receptor Trp64Arg, beta2 adrenergic receptor Arg16Gly and uncoupling protein $1-3826 \mathrm{~A}>\mathrm{G}$ polymorphisms detected by Smart Amplification Process 2. Endocr J 2011.

31. Jacene HA, Cohade CC, Zhang Z, Wahl RL. The Relationship between Patients' Serum Glucose Levels and Metabolically Active Brown Adipose Tissue Detected by PET/CT. Mol Imaging Biol 2011;13:1278-83.

32. Lee $\mathrm{P}$, Greenfield JR, Ho KK, Fulham MJ. A critical appraisal of the prevalence and metabolic significance of brown adipose tissue in adult humans. Am J Physiol Endocrinol Metab 2010;299:E601-6.

33. Skarulis MC, Celi FS, Mueller E, et al. Thyroid hormone induced brown adipose tissue and amelioration of diabetes in a patient with extreme insulin resistance. J Clin Endocrinol Metab 2010;95:256-62.

34. Orava J, Nuutila P, Lidell ME, et al. Different metabolic responses of human brown adipose tissue to activation by cold and insulin. Cell Metab 2011;14:272-9.

35. Goossens $\mathrm{GH}$, Bizzarri A, Venteclef $\mathrm{N}$, et al. Increased adipose tissue oxygen tension in obese compared with lean men is accompanied by insulin resistance, impaired adipose tissue capillarization, and inflammation. Circulation 2011;124:6776.

36. Bartelt A, Bruns OT, Reimer R, et al. Brown adipose tissue activity controls triglyceride clearance. Nat Med 2011;17:200-5.

37. Law MR, Wald NJ, Rudnicka AR. Quantifying effect of statins on low density lipoprotein cholesterol, ischaemic heart disease, and stroke: systematic review and meta-analysis. BMJ 2003;326:1423.

38. Farrell GC, Larter CZ. Nonalcoholic fatty liver disease: from steatosis to cirrhosis. Hepatology 2006;43:S99-S112.

39. Yilmaz $Y$, Ones $T$, Purnak $T$, et al. Association between the presence of brown adipose tissue and non-alcoholic fatty liver disease in adult humans. Aliment Pharmacol Ther 2011.

40. Labruna G, Pasanisi F, Nardelli C, et al. UCP1 -3826 AG+GG genotypes, adiponectin, and leptin/adiponectin ratio in severe obesity. J Endocrinol Invest 
2009;32:525-9.

41. Moller N, Nielsen S, Nyholm B, Porksen N, Alberti KG, Weeke J. Glucose turnover, fuel oxidation and forearm substrate exchange in patients with thyrotoxicosis before and after medical treatment. Clin Endocrinol (Oxf) 1996;44:453-9.

42. Lim VS, Zavala DC, Flanigan MJ, Freeman RM. Basal oxygen uptake: a new technique for an old test. J Clin Endocrinol Metab 1986;62:863-8.

43. al-Adsani H, Hoffer LJ, Silva JE. Resting energy expenditure is sensitive to small dose changes in patients on chronic thyroid hormone replacement. J Clin Endocrinol Metab 1997;82:1118-25.

44. Hadi $M$, Chen CC, Whatley $M$, Pacak $K$, Carrasquillo JA. Brown fat imaging with (18)F-6-fluorodopamine PET/CT, (18)F-FDG PET/CT, and (123)I-MIBG SPECT: a study of patients being evaluated for pheochromocytoma. J Nucl Med 2007;48:1077-83.

45. Kuji I, Imabayashi E, Minagawa A, Matsuda H, Miyauchi T. Brown adipose tissue demonstrating intense FDG uptake in a patient with mediastinal pheochromocytoma. Ann Nucl Med 2008;22:231-5.

46. Shellock FG, Riedinger MS, Fishbein MC. Brown adipose tissue in cancer patients: possible cause of cancer-induced cachexia. J Cancer Res Clin Oncol 1986;111:82-5.

47. Dobert N, Menzel C, Hamscho N, Wordehoff W, Kranert WT, Grunwald F. Atypical thoracic and supraclavicular FDG-uptake in patients with Hodgkin's and non-Hodgkin's lymphoma. Q J Nucl Med Mol Imaging 2004;48:33-8.

48. Bing $C$, Bao $Y$, Jenkins J, et al. Zinc-alpha2-glycoprotein, a lipid mobilizing factor, is expressed in adipocytes and is up-regulated in mice with cancer cachexia. Proc Natl Acad Sci U S A 2004;101:2500-5.

49. Arruda AP, Milanski M, Romanatto T, et al. Hypothalamic actions of tumor necrosis factor alpha provide the thermogenic core for the wastage syndrome in cachexia. Endocrinology 2010;151:683-94.

50. Furlong MA, Fanburg-Smith JC, Miettinen $M$. The morphologic spectrum of hibernoma: a clinicopathologic study of 170 cases. Am J Surg Pathol 2001;25:80914.

51. Cannon B, Houstek J, Nedergaard J. Brown adipose tissue. More than an effector of thermogenesis? Ann N Y Acad Sci 1998;856:171-87.

52. Nguyen KD, Qiu Y, Cui X, et al. Alternatively activated macrophages produce catecholamines to sustain adaptive thermogenesis. Nature 2011.

53. Scheller J, Chalaris A, Schmidt-Arras D, Rose-John S. The pro- and 
anti-inflammatory properties of the cytokine interleukin-6. Biochim Biophys Acta 2011;1813:878-88.

54. Vegiopoulos A, Muller-Decker K, Strzoda D, et al. Cyclooxygenase-2 controls energy homeostasis in mice by de novo recruitment of brown adipocytes. Science 2010;328:1158-61.

55. Petrovic N, Walden TB, Shabalina IG, Timmons JA, Cannon B, Nedergaard J. Chronic peroxisome proliferator-activated receptor gamma (PPARgamma) activation of epididymally derived white adipocyte cultures reveals a population of thermogenically competent, UCP1-containing adipocytes molecularly distinct from classic brown adipocytes. J Biol Chem 2009;285:7153-64.

56. David CM. Sudden infant death syndrome: a hypothesis. Med Hypotheses 1997;49:61-7.

57. Symonds ME, Bryant MJ, Clarke L, Darby CJ, Lomax MA. Effect of maternal cold exposure on brown adipose tissue and thermogenesis in the neonatal lamb. J Physiol 1992;455:487-502.

58. Hondares E, Rosell M, Gonzalez FJ, Giralt M, Iglesias R, Villarroya F. Hepatic FGF21 expression is induced at birth via PPARalpha in response to milk intake and contributes to thermogenic activation of neonatal brown fat. Cell Metab 2010;11:206-12.

59. Ouellet $\mathrm{V}$, Labbe SM, Blondin DP, et al. Brown adipose tissue oxidative metabolism contributes to energy expenditure during acute cold exposure in humans. J Clin Invest 2012;122:545-52.

60. Davis TR. Chamber cold acclimatization in man. J Appl Physiol 1961;16:10115.

61. Huttunen P, Hirvonen J, Kinnula V. The occurrence of brown adipose tissue in outdoor workers. Eur J Appl Physiol Occup Physiol 1981;46:339-45.

62. Sanchez-Alavez $M$, Tabarean IV, Osborn $O$, et al. Insulin causes hyperthermia by direct inhibition of warm-sensitive neurons. Diabetes 2010;59:4350.

63. Wu C, Cheng W, Xing H, Dang Y, Li F, Zhu Z. Brown adipose tissue can be activated or inhibited within an hour before 18F-FDG injection: a preliminary study with microPET. J Biomed Biotechnol 2011;2011:159834.

64. Wijers SL, Schrauwen $P$, van Baak MA, Saris WH, van Marken Lichtenbelt WD. Beta-adrenergic receptor blockade does not inhibit cold-induced thermogenesis in humans: possible involvement of brown adipose tissue. J Clin Endocrinol Metab 2011;96:E598-605.

65. Thomas C, Gioiello A, Noriega L, et al. TGR5-mediated bile acid sensing 
controls glucose homeostasis. Cell Metab 2009;10:167-77.

66. Thomas C, Auwerx J, Schoonjans K. Bile acids and the membrane bile acid receptor TGR5--connecting nutrition and metabolism. Thyroid 2008;18:167-74.

67. Watanabe $M$, Houten SM, Mataki $C$, et al. Bile acids induce energy expenditure by promoting intracellular thyroid hormone activation. Nature 2006;439:484-9.

68. Murphy KG, Bloom SR. Gut hormones and the regulation of energy homeostasis. Nature 2006;444:854-9.

69. Wynne K, Park AJ, Small CJ, et al. Oxyntomodulin increases energy expenditure in addition to decreasing energy intake in overweight and obese humans: a randomised controlled trial. Int J Obes (Lond) 2006;30:1729-36.

70. Thomas C, Pellicciari R, Pruzanski M, Auwerx J, Schoonjans K. Targeting bile-acid signalling for metabolic diseases. Nat Rev Drug Discov 2008;7:678-93.

71. Kajimura S, Seale P, Spiegelman BM. Transcriptional control of brown fat development. Cell Metab 2010;11:257-62.

72. Seale P, Conroe HM, Estall J, et al. Prdm16 determines the thermogenic program of subcutaneous white adipose tissue in mice. J Clin Invest 2011;121:96105.

73. Schulz TJ, Huang TL, Tran TT, et al. Identification of inducible brown adipocyte progenitors residing in skeletal muscle and white fat. Proc Natl Acad Sci U S A 2011;108:143-8.

74. Lee P, Swarbrick MM, Ting Zhao J, Ho KK. Inducible Brown Adipogenesis of Supraclavicular Fat in Adult Humans. Endocrinology 2011.

75. Bostrom $\mathrm{P}, \mathrm{Wu}$ J, Jedrychowski MP, et al. A PGC1-alpha-dependent myokine that drives brown-fat-like development of white fat and thermogenesis. Nature 2012;481:463-8. 


$$
\theta_{-1}
$$




\section{Chapter 3}

\section{Brown adipose tissue in morbidly obese subjects}

The contents of this chapter are published in Vijgen GH, Bouvy ND, Teule GJ, Brans B, Schrauwen P, van Marken Lichtenbelt WD. PLoS One. 2011 Feb 24;6(2):e17247. 


\section{Abstract}

\section{Background}

Cold-stimulated adaptive thermogenesis in brown adipose tissue (BAT) to increase energy expenditure is suggested as a possible therapeutic target for the treatment of obesity. We have recently shown high prevalence of BAT in adult humans, which was inversely related to body mass index (BMI) and body fat percentage (BF\%), suggesting that obesity is associated with lower BAT activity. Here, we examined BAT activity in morbidly obese subjects and its role in cold-induced thermogenesis (CIT) after applying a personalized cooling protocol. We hypothesize that morbidly obese subjects show reduced BAT activity upon cold exposure.

\section{Methods and Results}

After applying a personalized cooling protocol for maximal non-shivering conditions, BAT activity was determined using positron-emission tomography and computed tomography (PET-CT). Cold-induced BAT activity was detected in three out of 15 morbidly obese subjects. Combined with results from lean to morbidly obese subjects $(n=39)$ from previous study, the collective data show a highly significant correlation between BAT activity and body composition $(P<0.001)$, respectively explaining $64 \%$ and $60 \%$ of the variance in $\mathrm{BMI}(r=0.8 ; \mathrm{P}<0.001)$ and $\mathrm{BF} \%$ $(r=0.75 ; P<0.001)$. Obese individuals demonstrate a blunted CIT combined with low BAT activity. Only in BAT-positive subjects $(n=26)$ mean energy expenditure was increased significantly upon cold exposure $(51.5 \pm 6.7 \mathrm{~J} / \mathrm{s}$ versus $44.0 \pm 5.1 \mathrm{~J} / \mathrm{s}$, $\mathrm{P}=0.001$ ), and the increase was significantly higher compared to BAT-negative subjects $(+15.5 \pm 8.9 \%$ versus $+3.6 \pm 8.9 \%, P=0.001)$, indicating a role for BAT in CIT in humans.

\section{Conclusions}

This study shows that in an extremely large range of body compositions, BAT activity is highly correlated with $\mathrm{BMI}$ and $\mathrm{BF} \%$. BAT-positive subjects showed higher CIT, indicating that BAT is also in humans involved in adaptive thermogenesis. Increasing BAT activity could be a therapeutic target in (morbid) obesity. 


\section{Introduction}

Brown adipose tissue (BAT) is known for its capacity to generate heat in response to cold or diet to maintain thermal balance. The regulated production of heat is called adaptive thermogenesis. BAT is the main tissue for this adaptive thermogenesis in rodents and most likely in human infants. ${ }^{1,2}$ Although several early anatomical studies suggested that brown adipose tissue is present in adult humans ${ }^{3-5}$, its physiologic relevance was believed to be marginal for most. ${ }^{5,6}$ However, recent prospective, controlled studies showed that functional BAT is detectable in lean and obese adult humans after exposure to mild cold. ${ }^{7-9}$ We showed a high incidence of cold induced BAT activity that was inversely related to body mass index (BMI) and body fat percentage (BF\%). ${ }^{7}$ Other studies had similar findings. ${ }^{8-10}$

Cold-stimulated adaptive thermogenesis in BAT to increase energy expenditure is suggested as a possible therapeutic target for the treatment of obesity. ${ }^{11,12}$ Cold can indeed stimulate adaptive thermogenesis, but differences in body composition may be correlated with the magnitude and type of response. We showed that lean subjects increase energy expenditure significantly in response to mild cold, whereas obese subjects have a blunted cold-induced thermogenesis (CIT) and show a larger insulative response..$^{13}$ If BAT is directly responsible for CIT, the absence of CIT in obesity suggests that BAT is reduced or absent. In addition, if the presence of BAT is structurally lower in obesity, this could be a risk factor for the development of obesity. Not being able to burn off excess calories leads to a positive energy balance and predisposes to develop obesity. This could explain the differences in weight gain in classic overfeeding studies. ${ }^{14}$

In our previous report BAT abundance in obese subjects was relatively low, though detectable in most individuals. In the current study we address BAT activity in morbid obesity, a severe form of obesity characterized by a BMI $\geq 35 \mathrm{~kg} / \mathrm{m}^{2}$ with concomitant disease or $\geq 40 \mathrm{~kg} / \mathrm{m}^{2}$ without.

To ensure maximal non-shivering thermogenesis (NST), as occurs before the onset of shivering, ${ }^{15}$ every subject was exposed to an individualized cooling protocol. We hypothesized that morbidly obese in comparison to lean subjects show reduced BAT activity upon cold exposure. Furthermore, we examined the role of BAT activity in cold-induced thermogenesis in these morbidly obese subjects. 


\section{Methods}

The study was reviewed and approved by the medical ethics committee of the Maastricht University Medical Centre. Written informed consent was obtained from fifteen morbidly obese subjects, two male and thirteen female. The mean BMI was $42.1 \pm 3.8 \mathrm{~kg} / \mathrm{m}^{2}$ (range $\left.34.8-48.3 \mathrm{~kg} / \mathrm{m}^{2}\right)$, mean body fat percentage $48.5 \pm 4.5 \%$ (range 37.9-54.7\%), mean weight $123.9 \pm 16.7 \mathrm{~kg}$ (range 98.2-155.0 kg) and mean age 39.2 \pm 8.1 years (range $24-51$ years). Subjects were not included when diagnosed with diabetes or use of beta-blockers. One female subject used levothyroxin for hypothyroidism and was euthyroid for several years. One female subject used azathioprine and mesalazine for Crohn's disease. They were studied in the morning from 8 a.m. to 1 p.m. in a fasted state; only water consumption was allowed after 10 p.m. the night before measurements. During the measurements the subjects wore light standardized clothing (socks $0.02 \mathrm{clo}$, shirt 0.09 clo, sweatpants 0.28 clo, underwear 0.04 clo, total clo factor $0.43 \mathrm{clo}$ ). Core temperature was measured by a telemetric pill (CoreTemp, USA). This measurement failed in two subjects. Skin temperature was measured by wireless iButtons at the 14 ISO-defined skin sites. ${ }^{16}$ Subjects were placed in a specially equipped air permeable tent (Colorado altitude training, USA), which functioned as a transportable climate room at the department of nuclear medicine. The tent was cooled by an air-conditioning, which can be controlled to maintain the temperature inside the tent with an accuracy of $1^{\circ} \mathrm{C}$ [Kingma et al., submitted]. Subjects were placed in a semi-supine position in a nephrodialysis chair to lay comfortable during the personalized cooling protocol. Previous studies in our group showed high NST in lean subjects at the fixed temperature of $16^{\circ} \mathrm{C}$, without shivering..$^{17}$ However, in the current study group pilot experiments showed a lower onset temperature of shivering, with high interindividual variation. Therefore we used a personal cooling protocol to ensure maximal NST in the morbidly obese state. The level of cooling (temperature) was attuned to each individual, i.e. close to the cold level that induces shivering. This protocol was performed with subsequent FDG-PET-CT-imaging, because the intraindividual response to cold exposure shows high variation. ${ }^{17}$ In order to cool the dorsal site of the body, a water perfused cooling mattress was used (cooling device; Blankett roll, Cincinatti sub zero 2000, USA). Energy expenditure was measured for three hours by indirect calorimetry (Oxycon, Jaeger, Germany). One hour in thermoneutral conditions was followed by two hours of personalized cooling. In the first hour the room temperature was $22.3 \pm 0.4^{\circ} \mathrm{C}$ (baseline), followed by the second hour where the temperature of the room and cooling mattress were 
decreased until subjects subjectively reported shivering. This was confirmed by continuous measurement of muscle activity (pectoralis major) by use of on-skin electrodes attached to an electromyograph (Nicolet Viking, Nicolet Biomedical Inc, USA). When shivering occurred, the air and water temperatures were increased by steps of $1^{\circ} \mathrm{C}$ until shivering just stopped. In this manner NST was maximized for each individual without shivering. Stable cooling conditions were reached within $28.6 \pm 11.8$ minutes. After one hour of cold exposure $74 \mathrm{MBq}$ of ${ }^{18}$-FluoroDeoxy-Glucose (FDG) was injected. Cold exposure was maintained for another hour. To exclude the artifact of muscle activity, subjects were instructed to lay still. After this hour subjects were transferred to the positron emission tomography and computed tomography scanner (PET-CT-scanner) (Gemini TF PET-CT, Philips, the Netherlands). The scanning protocol and data interpretation were identical to our previous study protocol. ${ }^{7}$ Energy expenditure, room temperatures and body temperatures were sampled on a one minute basis. On a separate occasion body composition was measured with a dual $x$-ray absorptiometry (DXA, Hologic, type Discovery A, USA). Statistical analysis was performed with PASW Statistics 18.0 for Mac OS $X$ 10.6.4. Reported data is expressed as means $\pm S D$. Total BAT activity is expressed in kiloBequerel $(\mathrm{kBq})$. To compare findings before and after cold exposure paired t-tests were used; unpaired t-tests were used to compare BAT-positive to BAT-negative subjects. To identify correlations between variables linear regression analyses were conducted. For the relation between brown-adipose-tissue activity and body composition, exponential curve fitting was applied.

\section{Results}

\section{Individual cooling conditions}

Temperature conditions before the onset of shivering were variable. Stable cooling temperatures were established in fourteen subjects (room temperature $14.7 \pm 2.4^{\circ} \mathrm{C}$, inlet temperature of cooling mattress $13.3 \pm 4.7^{\circ} \mathrm{C}$ ). One female subject (BMI $38.7 \mathrm{~kg} / \mathrm{m}^{2}$, body fat $49.9 \%$, age 44 years, BAT $0 \mathrm{kBq}$ ) did not report shivering subjectively nor electromyographically at the lowest possible room $\left(12^{\circ} \mathrm{C}\right)$ and cooling mattress (inlet temperature of water mattress $4^{\circ} \mathrm{C}$ ) conditions. 


\section{BAT activity}

In the current morbidly obese group mean BAT activity was low $(5.5 \pm 14.8 \mathrm{kBq}$, range $0.0-54.5 \mathrm{kBq}$ ). Only three female subjects showed a cold induced increased FDG uptake in the neck and supraclavicular area on FDG-PET-CT-imaging (Figure 1). In these three subjects, the BAT activity was $4.48 \mathrm{kBq}\left(\mathrm{BMI} 42.5 \mathrm{~kg} / \mathrm{m}^{2}\right.$, body fat $51.2 \%$, age 47 years), $23.61 \mathrm{kBq}$ (BMI $36.9 \mathrm{~kg} / \mathrm{m}^{2}$, body fat $50.8 \%$, age 47 years) and $54.45 \mathrm{kBq}$ (BMI $46.3 \mathrm{~kg} / \mathrm{m}^{2}$, body fat $53.7 \%$, age 32 years). No BAT activity was detected in the other 12 subjects.

\section{Energy expenditure}

On average, energy expenditure during cooling compared to baseline did not increase significantly (baseline $41.9 \pm 3.3 \mathrm{~J} / \mathrm{s}$, mild cold $43.7 \pm 4.8 \mathrm{~J} / \mathrm{s}, \mathrm{P}=0.100$ ) (Table 1). However, a large interindividual variation was evident. The calorimetry measurement in one female subject failed and was excluded from analysis.

\section{Core and skin temperature}

During cooling we observed a significant rise in core temperature $\left(37.2 \pm 0.4^{\circ} \mathrm{C}\right.$ in thermoneutral conditions $(\mathrm{TN})$ versus $37.5 \pm 0.3^{\circ} \mathrm{C}$ during cold exposure $\left(\mathrm{T}_{\text {cold }}\right)$, $\mathrm{P}=0.048)$ and a drop in mean skintemperature $\left(T N 31.7 \pm 0.6^{\circ} \mathrm{C}\right.$ versus $\mathrm{T}_{\text {cold }}$ $27.7 \pm 1.5^{\circ} \mathrm{C}, \mathrm{P}<0.001$ ) (Table 1). Compared to thermoneutral conditions, cooling significantly increased the core-mean skin temperature gradient (TN $5.6 \pm 0.6^{\circ} \mathrm{C}$ versus $\mathrm{T}_{\text {Cold }} 9.9 \pm 1.6^{\circ} \mathrm{C}, \mathrm{P}<0.001$ ) (Table 1 ).

\section{Relationship of BAT - BMI}

When combining the data from the current and our previous study, ${ }^{7}$ we found strong correlations between BAT activity and BMI ( $r=0.80, P<0.001)$, BAT activity and body fat mass $(r=0.80, P<0.001)$, and BAT activity and BF\% $(r=0.75, P<0.001)$ (Figure 2).

In the combined group there were 26 subjects that showed active BAT on cold exposure, whereas 13 subjects did not. We therefore reanalysed our energy expenditure data in BAT-positive and BAT-negative subjects (age: 26.1 7.1 yrs, range 18-47 yrs versus 37.1 \pm 9.4 yrs, range 20-51 yrs, $P=0.001$, and body fat $\%: 25.9 \pm 11.9 \%$ versus $47.2 \pm 4.7 \%, P<0.001$ in BAT+ vs BAT- resp.). There was no difference in fat free mass, an important contributor to resting metabolic rate $(R M R)$, between both groups $(68.6 \pm 9.9 \mathrm{~kg}$ versus $64.2 \pm 9.8 \mathrm{~kg}, \mathrm{P}=0.195)$. 
RMR under thermoneutral conditions did not differ between BAT-positive and BAT-negative subjects $(44.6 \pm 5.2 \mathrm{~J} / \mathrm{s}$ versus $42.4 \pm 3.3 \mathrm{~J} / \mathrm{s}, \mathrm{P}=0.123)$, but after cold exposure BAT-positive subjects showed a higher energy expenditure $(51.5 \pm 6.7 \mathrm{~J} / \mathrm{s}$ versus $44.0 \pm 5.1 \mathrm{~J} / \mathrm{s}, \mathrm{P}=0.001$ ). Very interestingly, mean energy expenditure after cold exposure increased significantly within the BAT-positive group, but not within the BAT-negative group $(+15.5 \pm 8.9 \%$ versus $+3.6 \pm 8.9 \%, P=0.001$, Figure $2 \mathrm{C})$.

In the BAT-negative group the core temperature was higher, both in thermoneutral conditions $\left(37.1 \pm 0.4^{\circ} \mathrm{C}\right.$ versus $36.7 \pm 0.4^{\circ} \mathrm{C}, \mathrm{P}=0.018$ ) and after cold exposure ( $37.4 \pm 0.4^{\circ} \mathrm{C}$ versus $36.9 \pm 0.3^{\circ} \mathrm{C}, \mathrm{P}=0.001$ ). However, mean skin temperatures did not show a significant difference in thermoneutral conditions $\left(31.9 \pm 0.6^{\circ} \mathrm{C}\right.$ versus $\left.32.3 \pm 0.7^{\circ} \mathrm{C}, \mathrm{P}=0.076\right)$, nor during cold exposure $\left(28.0 \pm 1.2^{\circ} \mathrm{C}\right.$ versus $28.6 \pm 1.2^{\circ} \mathrm{C}$, $\mathrm{P}=0.135)$. The gradient between core temperature and mean skin temperature was larger in the BAT-negative group in thermoneutral conditions $\left(5.3 \pm 0.5^{\circ} \mathrm{C}\right.$ versus $\left.4.5 \pm 0.8^{\circ} \mathrm{C}, \mathrm{P}=0.004\right)$ and during cold exposure $\left(9.6 \pm 1.5^{\circ} \mathrm{C}\right.$ versus $8.3 \pm 1.4^{\circ} \mathrm{C}$, $\mathrm{P}=0.023)$. The gradient between core temperature and mean skin temperature was larger in the BAT-negative group in thermoneutral conditions $\left(5.3 \pm 0.5^{\circ} \mathrm{C}\right.$ versus $\left.4.5 \pm 0.8^{\circ} \mathrm{C}, \mathrm{P}=0.004\right)$ and during cold exposure $\left(9.6 \pm 1.5^{\circ} \mathrm{C}\right.$ versus $8.3 \pm 1.4^{\circ} \mathrm{C}$, $\mathrm{P}=0.023)$. 
Table 1. Energy expenditure, body core temperature, mean skin temperature and core-skin temperature gradient in thermoneutral conditions (TN) and during mild cold (Cold) in morbidly obese subjects.

\begin{tabular}{lccccccc} 
& TN & Min & Max & Cold & Min & Max & P value \\
\hline Energy expenditure $(\mathrm{J} / \mathrm{s})$ & $41.9 \pm 3.3$ & 37.9 & 49.6 & $43.7 \pm 4.8$ & 35.7 & 51.1 & 0.100 \\
Body core temperature $\left({ }^{\circ} \mathrm{C}\right)$ & $37.2 \pm 0.4$ & 36.5 & 37.8 & $37.5 \pm 0.3$ & 36.9 & 37.9 & 0.048 \\
Mean skin temperature $\left({ }^{\circ} \mathrm{C}\right)$ & $31.7 \pm 0.6$ & 30.4 & 32.9 & $27.7 \pm 1.5$ & 25.2 & 29.6 & $<0.001$ \\
Core-mean skin gradient $\left({ }^{\circ} \mathrm{C}\right)$ & $5.6 \pm 0.6$ & 4.9 & 6.7 & $9.9 \pm 1.6$ & 7.3 & 12.2 & $<0.001$
\end{tabular}
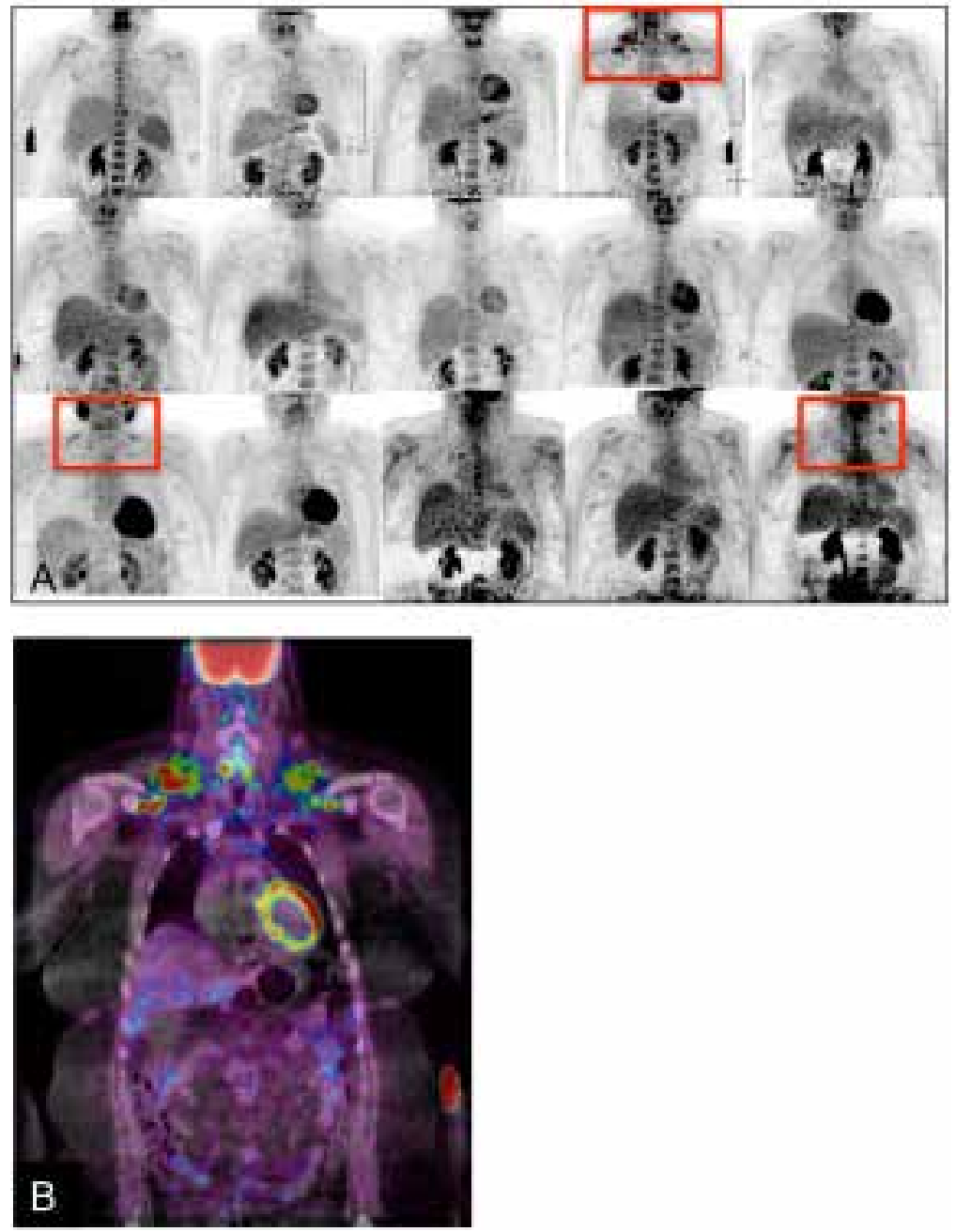

Figure 1. PET-images of fifteen morbidly obese subjects. Three female subjects showed BAT activity in the supraclavicular area, highlighted by a red rectangle (A). Combined PET- and CT-imaging shows FDG-uptake in supraclavicular adipose tissue (this was the morbidly obese subject that showed the most BAT activity) (B). 

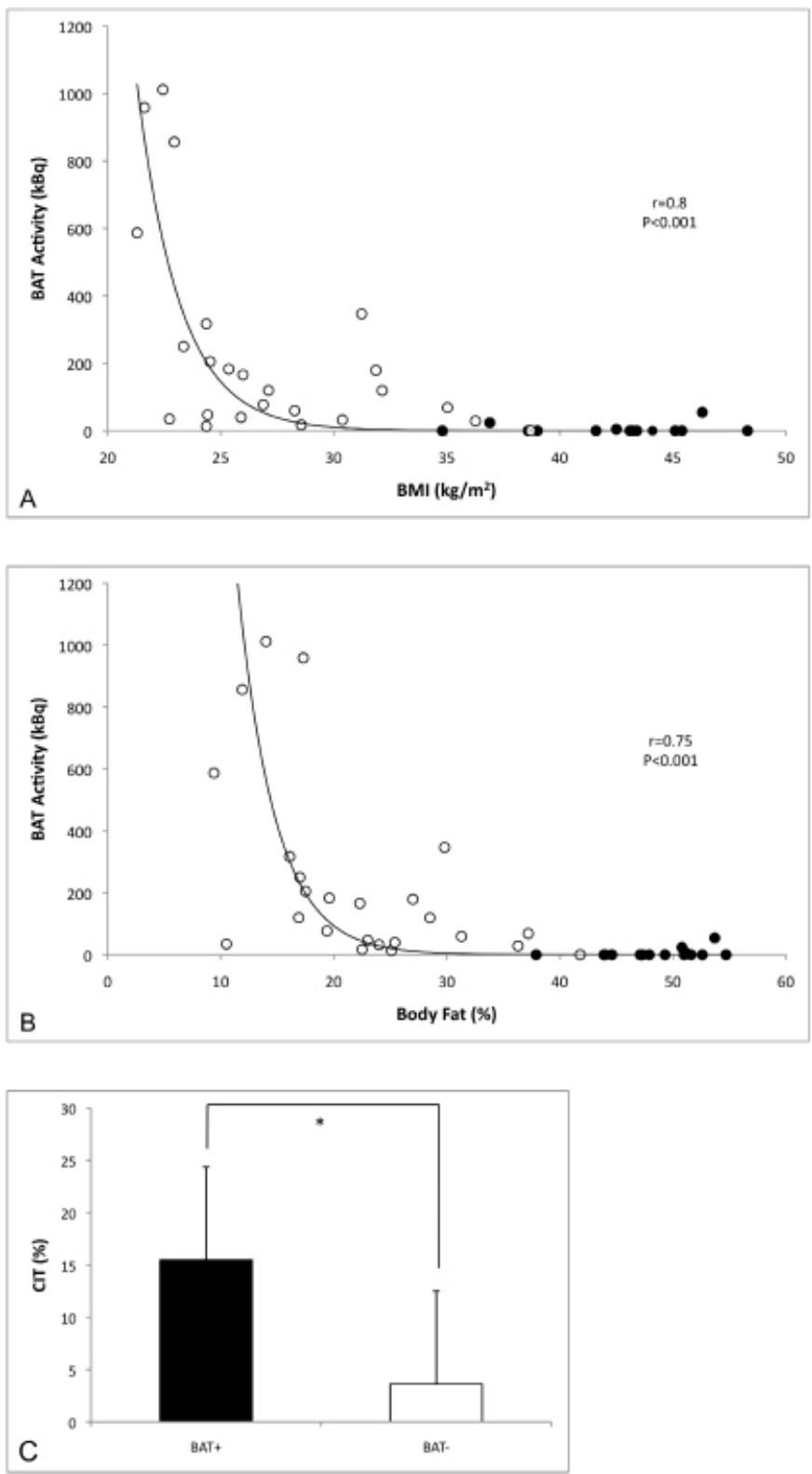

Figure 2. Brown adipose tissue activity in relation to body mass index (A) and body fat percentage (B). The black dots indicate the current study group, the open dots indicate previously performed measurements. Cold-induced thermogenesis (CIT), denoted in percentages, is significantly increased in 26 BAT-positive $(\mathrm{BAT}+)$ compared to 13 BAT-negative (BAT-) subjects $(\mathrm{C}){ }^{*}$ : $\mathrm{P}<0.05$ 
Very interestingly, mean energy expenditure after cold exposure increased significantly within the BAT-positive group, but not within the BAT-negative group ( $+15.5 \pm 8.9 \%$ versus $+3.6 \pm 8.9 \%, P=0.001$, Figure $2 C$ ).

In the BAT-negative group the core temperature was higher, both in thermoneutral conditions $\left(37.1 \pm 0.4^{\circ} \mathrm{C}\right.$ versus $\left.36.7 \pm 0.4^{\circ} \mathrm{C}, \mathrm{P}=0.018\right)$ and after cold exposure $\left(37.4 \pm 0.4^{\circ} \mathrm{C}\right.$ versus $\left.36.9 \pm 0.3^{\circ} \mathrm{C}, \mathrm{P}=0.001\right)$. However, mean skin temperatures did not show a significant difference in thermoneutral conditions $\left(31.9 \pm 0.6^{\circ} \mathrm{C}\right.$ versus $\left.32.3 \pm 0.7^{\circ} \mathrm{C}, \mathrm{P}=0.076\right)$, nor during cold exposure $\left(28.0 \pm 1.2^{\circ} \mathrm{C}\right.$ versus $28.6 \pm 1.2^{\circ} \mathrm{C}$, $\mathrm{P}=0.135)$. The gradient between core temperature and mean skin temperature was larger in the BAT-negative group in thermoneutral conditions $\left(5.3 \pm 0.5^{\circ} \mathrm{C}\right.$ versus $\left.4.5 \pm 0.8^{\circ} \mathrm{C}, \mathrm{P}=0.004\right)$ and during cold exposure $\left(9.6 \pm 1.5^{\circ} \mathrm{C}\right.$ versus $8.3 \pm 1.4^{\circ} \mathrm{C}$, $\mathrm{P}=0.023)$.

\section{Discussion}

In this study active BAT was detected in only three out of fifteen morbidly obese patients, suggesting that morbid obesity is associated with low BAT activity. On the other hand, the data also demonstrate that even in this group BAT is present in some subjects and can be activated by cold exposure. Nevertheless, the combined results of morbidly obese subjects with those of previously reported lean and obese subjects, clearly show that body composition is highly related to BAT activity. This is in line with lower UCP-1-presence in the intraperitoneal adipose tissue of morbidly obese compared to lean subjects. ${ }^{18}$

A limitation in our analysis is fact that our previous study was composed of male subjects, whereas the morbidly obese subjects were mostly females. However, retrospective studies have actually shown higher BAT activity in women, ${ }^{19,20}$ which would only further strengthen the conclusion that BAT activity is reduced in obesity. The obese subjects generally show a high insulative response. Therefore, it is important to note that we used an individualized cold exposure protocol that achieves maximal non-shivering conditions. Despite this approach, the major part of obese and morbidly obese subjects did not show BAT activation. In the three BAT-positive morbidly obese subjects, two subjects showed pronounced CIT (BAT $23.61 \mathrm{kBq}$; CIT 18.76\%, BAT $4.48 \mathrm{kBq}$; CIT 6.04\%). Unfortunately, in the third subject (BAT $54.45 \mathrm{kBq}$ ) measurement of energy expenditure failed for technical reasons. However, CIT in the two BAT positive morbidly obese subjects was high compared to the average of the morbidly obese group (mean CIT $4.45 \pm 9.33 \%$ ). This suggests a possible relation between active BAT after cold exposure and CIT. 
Since approximately two-thirds of the total group of subjects showed cold-induced BAT activity whereas one-third did not, we further tested the hypothesis that BAT-positive subjects would have higher (cold-induced) thermogenesis. Interestingly, subjects that have active BAT also have a significant increase in energy expenditure during cold exposure, whereas cold exposure did not increase energy expenditure in BAT-negative subjects. Although age is significantly different in BAT-positive and BAT-negative subjects, the range between both groups (18-47 yrs versus 20-51 yrs) strongly overlaps, with active and inactive BAT spread over all age quartiles.

After we had previously shown that BAT activity was related to RMR, the additional analysis in this study shows that BAT activity may in fact be involved in adaptive thermogenesis. The role of FFM in this process can be excluded, since there is no significant difference in FFM between the analyzed groups. Therefore, the data suggest that - like in rodents - BAT is indeed involved in cold-induced thermogenesis in humans. This is in line with earlier findings in small study groups. ${ }^{10}$

The main native BAT depot is located supraclavicular. ${ }^{3}$ That depot shows FDG-uptake after cold exposure in all subjects with active BAT. Biopsies taken from this region show typical BAT cells, mostly surrounded by white fat cells. ${ }^{8,21}$ A pure BAT depot, as seen in rodents, is rare in adult man. Next to native BAT, recently 'brite' or 'beige' cells derived from white adipose tissue were reported. ${ }^{22-25}$ These cells stem from a different cell lineage than native BAT, but they possess the ability to generate heat by mitochondrial uncoupling similar to native BAT cells.

Most morbidly obese subjects in this study had no detectable BAT activity after cold exposure. From our study, it cannot be concluded that the lack of BAT is cause or consequence of severe obesity in our subjects. However, when the amount of native BAT is minimal or absent, this could lead to severe overweight. For example, mice that lack UCP-1, essential for mitochondrial uncoupling in BAT, become obese when studied under thermoneutral conditions. ${ }^{26}$ With a congenital shortage of native BAT, brite cells could therefore be of great therapeutic interest.

On the other hand, in adult man a high amount of thermogenic potential can be present throughout the body, but may remain dormant when insulation makes a metabolic response unnecessary, as may be the case in the morbidly obese state. Indeed, prolonged cold exposure in rodents and rhesus monkeys increases the amount of (brite) BAT, supporting the idea of recruitable BAT. ${ }^{1}$ It would therefore be interesting to test if BAT can be recruited in morbidly obese subjects. Next to prolonged cold exposure, other therapeutic targets could recruit BAT: a) weight reduction decreases the insulative capacity that blunts CIT, b) pharmacological intervention, such as sympathicomimetics has a high potential and c) implantation 
of cultured brown adipocytes could all counteract severe overweight.12,27 In conclusion, this study shows that in an extremely large range of body compositions, BAT activity is highly correlated with $\mathrm{BMI}$ and $\mathrm{BF} \%$. Active BAT was seen in three out of 15 morbidly obese subjects. BAT-positive subjects showed higher CIT, indicating that BAT is also in humans involved in adaptive thermogenesis. 


\section{References}

1. Cannon B, Nedergaard J. Brown adipose tissue: function and physiological significance. Physiol Rev 2004;84:277-359.

2. Enerback S. Human brown adipose tissue. Cell Metab 2010;11:248-52.

3. Heaton JM. The distribution of brown adipose tissue in the human. J Anat 1972;112:35-9.

4. Huttunen P, Hirvonen J, Kinnula V. The occurrence of brown adipose tissue in outdoor workers. Eur J Appl Physiol Occup Physiol 1981;46:339-45.

5. Astrup A, Bulow J, Madsen J, Christensen NJ. Contribution of BAT and skeletal muscle to thermogenesis induced by ephedrine in man. Am J Physiol 1985;248:E507-15.

6. Himms-Hagen J. Does brown adipose tissue (BAT) have a role in the physiology or treatment of human obesity? Rev Endocr Metab Disord 2001;2:395401.

7. van Marken Lichtenbelt WD, Vanhommerig JW, Smulders NM, et al. Cold-activated brown adipose tissue in healthy men. N Engl J Med 2009;360:15008 .

8. Virtanen KA, Lidell ME, Orava J, et al. Functional brown adipose tissue in healthy adults. N Engl J Med 2009;360:1518-25.

9. Saito $M$, Okamatsu-Ogura $Y$, Matsushita $M$, et al. High Incidence of Metabolically Active Brown Adipose Tissue in Healthy Adult Humans: Effects of Cold Exposure and Adiposity. Diabetes 2009;58:1526-31.

10. Yoneshiro T, Aita S, Matsushita M, et al. Brown Adipose Tissue, WholeBody Energy Expenditure, and Thermogenesis in Healthy Adult Men. Obesity (Silver Spring) 2011;19:13-6.

11. Lidell ME, Enerback $S$. Brown adipose tissue-a new role in humans? Nature Reviews Endocrinology 2010;6:319-25.

12. Tseng $\mathrm{YH}$, Cypess $\mathrm{AM}$, Kahn CR. Cellular bioenergetics as a target for obesity therapy. Nat Rev Drug Discov 2010;9:465-82.

13. Wijers SL, Saris WH, van Marken Lichtenbelt WD. Cold-induced adaptive thermogenesis in lean and obese. Obesity (Silver Spring) 2010;18:1092-9.

14. Bouchard C, Tremblay A, Despres JP, et al. The response to long-term overfeeding in identical twins. N Engl J Med 1990;322:1477-82.

15. van Ooijen $A M$, van Marken Lichtenbelt WD, van Steenhoven $A A$, Westerterp KR. Cold-induced heat production preceding shivering. $\mathrm{Br} J \mathrm{Nutr}$ 2005;93:387-91. 
16. van Marken Lichtenbelt WD, Daanen HA, Wouters $L$, et al. Evaluation of wireless determination of skin temperature using iButtons. Physiol Behav 2006;88:489-97.

17. van Ooijen $A M$, van Marken Lichtenbelt WD, van Steenhoven $A A$, Westerterp KR. Seasonal changes in metabolic and temperature responses to cold air in humans. Physiol Behav 2004;82:545-53.

18. Oberkofler H, Dallinger G, Liu YM, Hell E, Krempler F, Patsch W. Uncoupling protein gene: quantification of expression levels in adipose tissues of obese and non-obese humans. J Lipid Res 1997;38:2125-33.

19. Cypess AM, Lehman S, Williams $G$, et al. Identification and importance of brown adipose tissue in adult humans. N Engl J Med 2009;360:1509-17.

20. Pfannenberg C, Werner MK, Ripkens S, et al. Impact of age on the relationships of brown adipose tissue with sex and adiposity in humans. Diabetes 2010;59:1789-93.

21. Zingaretti MC, Crosta F, Vitali A, et al. The presence of UCP1 demonstrates that metabolically active adipose tissue in the neck of adult humans truly represents brown adipose tissue. FASEB J 2009;23:3113-20.

22. Ishibashi J, Seale P. Beige can be slimming. Science 2010;328:1113-4.

23. Petrovic N, Walden TB, Shabalina IG, Timmons JA, Cannon B, Nedergaard J. Chronic peroxisome proliferator-activated receptor gamma (PPARgamma) activation of epididymally derived white adipocyte cultures reveals a population of thermogenically competent, UCP1-containing adipocytes molecularly distinct from classic brown adipocytes. J Biol Chem 2009;285:7153-64.

24. Barbatelli G, Murano I, Madsen L, et al. The emergence of cold-induced brown adipocytes in mouse white fat depots is determined predominantly by white to brown adipocyte transdifferentiation. Am J Physiol Endocrinol Metab 2010;298:E1244-53.

25. Frontini A, Cinti S. Distribution and development of brown adipocytes in the murine and human adipose organ. Cell Metab 2010;11:253-6.

26. Feldmann HM, Golozoubova V, Cannon B, Nedergaard J. UCP1 ablation induces obesity and abolishes diet-induced thermogenesis in mice exempt from thermal stress by living at thermoneutrality. Cell Metab 2009;9:203-9.

27. Ghorbani M, Himms-Hagen J. Appearance of brown adipocytes in white adipose tissue during CL 316,243-induced reversal of obesity and diabetes in Zucker $\mathrm{fa} / \mathrm{fa}$ rats. Int J Obes Relat Metab Disord 1997;21:465-75. 


$$
\theta_{-1}
$$




\section{Chapter 4}

\section{Increase in brown adipose tissue activity after weight loss in morbidly obese subjects}

The contents of this chapter are published in Vijgen GH, Bouvy ND, Teule GJ, Brans B, Hoeks J, Schrauwen P, van Marken Lichtenbelt WD. Journal of Clinical Endocrinology and Metabolism. 2012 Jul;97(7):E1229-33. 


\section{Abstract}

\section{Background}

Stimulation of thermogenesis in brown adipose tissue (BAT) is a potential target to treat obesity. We earlier demonstrated that BAT activity is relatively low in obese subjects. It is unknown whether BAT can be recruited in adult humans.

\section{Methods}

Ten morbidly obese subjects eligible for laparoscopic adjustable gastric banding (LAGB) surgery were studied before and one year after bariatric surgery. The main outcome measure was BAT activity, as determined after acute cold stimulation using ${ }^{18} \mathrm{~F}$-fluoro-deoxyglucose positron-emission-tomography and computedtomography (FDG-PET-CT).

\section{Results}

Before surgery, only two out of 10 subjects showed active BAT. One year after surgery the number of subjects with active BAT was increased to five. After weight loss, BAT-positive subjects had significantly higher non-shivering thermogenesis (NST) compared to BAT-negative subjects $(\mathrm{P}<0.05)$.

\section{Conclusions}

The results show that in humans BAT can be recruited in the regions where it was also reported in lean subjects before. These results for the first time show recruitment of BAT in humans and may open the door for BAT targeted treatments of obesity. 


\section{Introduction}

Until very recently, the role of brown adipose tissue (BAT) in human physiology was only considered to be of importance during the first years of life. However, metabolic imaging techniques (18-Fluoro-Deoxy-Glucose-Positron-EmissionTomography-and-Computed-Tomography $\left.\left({ }^{18} \mathrm{~F}-\mathrm{FDG}-\mathrm{PET}-\mathrm{CT}\right)\right)$ showed functionally active BAT upon cold exposure in adult man $\cdot^{1-4}$ We previously found that BAT activity is inversely related to body mass index (BMI) and body fat percentage (BF\%). ${ }^{2}$ This could suggest that a low level of BAT activity may predispose to obesity, when assumed that BAT activity is an intrinsic characteristic of an individual. Alternatively, low BAT activity may be an adaptive trait of obesity - for example due to improved insulation - which would predict that functional BAT could be re-recruited when body composition would change, for example after significant weight loss. Post-obese subjects indeed report an increased cold sensation during weight loss by bariatric surgery. ${ }^{5}$, personal observations It is still unknown if BAT can be recruited in man. However, the principle of recruitable BAT has been observed after prolonged cold exposure in rodents and primates. ${ }^{6}$ In man, seasonal variation influences BAT activity within subjects. ${ }^{3,6}$ In vitro stimulation of white adipose tissue (WAT) with peroxisome proliferator-activated-receptor-gamma (PPAR-gamma) induced the development of thermogenic 'brite' adipocytes.? Moreover, treating preadipocytes isolated from human subcutaneous adipose tissue with bone-morphogenic-protein-7 (BMP7) showed brown adipogenesis. ${ }^{8}$ Based on these observations, BAT recruitment in man seems feasible but has not yet been shown. Therefore, we investigated BAT activity in ten morbidly obese patients before and one year after laparoscopic adjustable gastric banding (LAGB) surgery as a model for caloric restriction.

The increase in energy expenditure that is observed upon mild cold exposure in absence of shivering in skeletal muscle is defined as non-shivering thermogenesis (NST). ${ }^{9}$ Active BAT in adult man is suggested to contribute to NST. Indeed, subjects with active BAT show significantly higher NST than subjects without BAT activity. ${ }^{10,11}$ Therefore we also studied NST in our subjects. 


\section{Methods}

Approval was obtained from the local ethical commission. Written informed consent was received from two male and eight female morbidly obese subjects, with a mean $\mathrm{BMI}$ of $41.7 \pm 4.4 \mathrm{~kg} / \mathrm{m}$ and a body mass of $127.0 \pm 17.7 \mathrm{~kg}$. One female subject used levothyroxin for hypothyroidism and was euthyroid for several years. Diabetes mellitus and use of beta-blockers were strict exclusion criteria.

\section{Study protocol}

All measurements took place several weeks before surgery between 8 a.m. to 1 p.m. under overnight fasted conditions. In the morning, subjects put on light standardized clothing (socks 0.02 clo, shirt 0.09 clo, sweatpants 0.28 clo, underwear 0.04 clo, total clo factor $0.43 \mathrm{clo}^{12}$ ). Body composition (body fat $\%$, fat mass (FM), fat free mass (FFM)) was determined by dual x-ray absorptiometry (DXA, Hologic, type Discovery A, USA). To determine body core temperature, a telemetric pill was orally ingested (CoreTemp, USA). Skin temperature was measured by applying iButtons at 14 ISO-defined skin sites. ${ }^{13}$ Next, subjects were placed in a specially adapted climate controlled tent (Colorado altitude training, USA). The temperature inside the tent was regulated by an air conditioner (accuracy of tent air temperature: $\pm 0.5^{\circ} \mathrm{C}$ ). Energy expenditure was measured by indirect calorimetry using a ventilated hood system (Oxycon, Jaeger, Germany).

\section{Personal cooling protocol and 18F-FDG-PET-CT}

In all subjects we performed the personal cooling protocol described earlier. ${ }^{10}$ In short, subjects were placed in the climate tent. They rested semi-supine on a nephrodialysis chair, covered with a water-perfused mattress. To ensure maximal non-shivering thermogenesis, every subject was cooled until they reported shivering. Shivering was confirmed by electromyography of the $\mathrm{m}$. pectoralis major. Upon the first signs of shivering the temperature was raised slightly (on average $1.8 \pm 0.6^{\circ} \mathrm{C}$ ) to insure only non-shivering thermogenesis (NST). One hour of measuring under thermoneutral conditions (room temperature before weight loss; $22.8 \pm 0.6^{\circ} \mathrm{C}$, after weight loss; $23.7 \pm 0.9^{\circ} \mathrm{C}, \mathrm{P}=0.079$, t0-t60) was followed by two hours of individual mild cold (t60-t180). Laser Doppler Flowmetry (Perimed PF5000, Sweden) was used to determine if a subject was in its thermoneutral zone. ${ }^{14}$ At the end of the first hour of cooling (t120), 10ml FDG was injected. After the second hour of cooling 
Table 1A. Subject characteristics in ten patients (two male, eight female) with measurements before and after weight loss. BMI indicates Body Mass Index.

\begin{tabular}{lccc} 
& Before & After & P-value \\
\hline Age $(\mathrm{yr})$ & $40 \pm 9$ & $41 \pm 9$ & \\
BMI $\left(\mathrm{kg} / \mathrm{m}^{2}\right)$ & $41.7 \pm 4.4$ & $29.8 \pm 4.2$ & $<0.001$ \\
Body mass $(\mathrm{kg})$ & $127.0 \pm 17.7$ & $90.8 \pm 16.7$ & $<0.001$ \\
Body fat $(\%)$ & $48.6 \pm 5.2$ & $34.8 \pm 7.9$ & $<0.001$ \\
Fat mass $(\mathrm{kg})$ & $62.1 \pm 10.9$ & $32.4 \pm 10.5$ & $<0.001$ \\
Fat free mass $(\mathrm{kg})$ & $63.5 \pm 11.7$ & $57.7 \pm 12.2$ & $<0.001$
\end{tabular}

Table 1B. Total brown adipose tissue (BAT) activity before and after weight loss. Group values $(\mathrm{n}=10)$ of BAT activity in kiloBequerel $(\mathrm{kBq})$, BAT volume $\left(\mathrm{cm}^{3}\right)$, average BAT activity $\left(\mathrm{kBq} / \mathrm{cm}^{3}\right)$ and total and maximal Standard Uptake Value (SUV ${ }^{\text {Total }}$, SUV ${ }^{\mathrm{Max}}$ ). In addition, values are shown for the group of five subjects that were indicated as BAT-positive (BAT+) based on their BAT-presence after weight loss.

\begin{tabular}{lcccccc} 
& \multicolumn{2}{c}{ Before } & \multicolumn{2}{c}{ After } & \multicolumn{2}{c}{ P-value } \\
& Group & BAT+ & Group & BAT+ & Group & BAT+ \\
\hline BAT activity $(\mathrm{kBq})$ & $7.8 \pm 18.0$ & $15.6 \pm 24.0$ & $51.5 \pm 87.8$ & $103.0 \pm 103.6$ & 0.147 & 0.153 \\
BAT volume $\left(\mathrm{cm}^{3)}\right.$ & $7.1 \pm 16.2$ & $14.2 \pm 21.6$ & $42.5 \pm 71.6$ & $85.0 \pm 83.9$ & 0.148 & 0.154 \\
BAT activity $\left(\mathrm{kBq} / \mathrm{cm}^{3}\right)$ & $0.2 \pm 0.5$ & $0.4 \pm 0.6$ & $0.6 \pm 0.6$ & $1.1 \pm 0.1$ & 0.074 & 0.062 \\
SUV Total & $30.3 \pm 72.4$ & $60.6 \pm 97.5$ & $118.0 \pm 210.6$ & $236.0 \pm 255.0$ & 0.238 & 0.259 \\
SUVMax & $1.4 \pm 3.0$ & $2.8 \pm 3.9$ & $3.1 \pm 3.9$ & $6.3 \pm 3.2$ & 0.197 & 0.212
\end{tabular}

${ }^{18} \mathrm{~F}-\mathrm{FDG}$-PET-CT-imaging was performed to quantify metabolically active BAT. The scanning protocol, FDG activity $(74 \mathrm{MBq})$ and data analysis were identical to our previous study. ${ }^{2}$

\section{Follow-up}

All subjects underwent LAGB without any perioperative or postoperative complications. One year after the LAGB procedure, all measurements were repeated.

\section{Statistical analysis}

Total BAT activity was expressed in kiloBequerel $(\mathrm{kBq})$ and Standard Uptake Value 

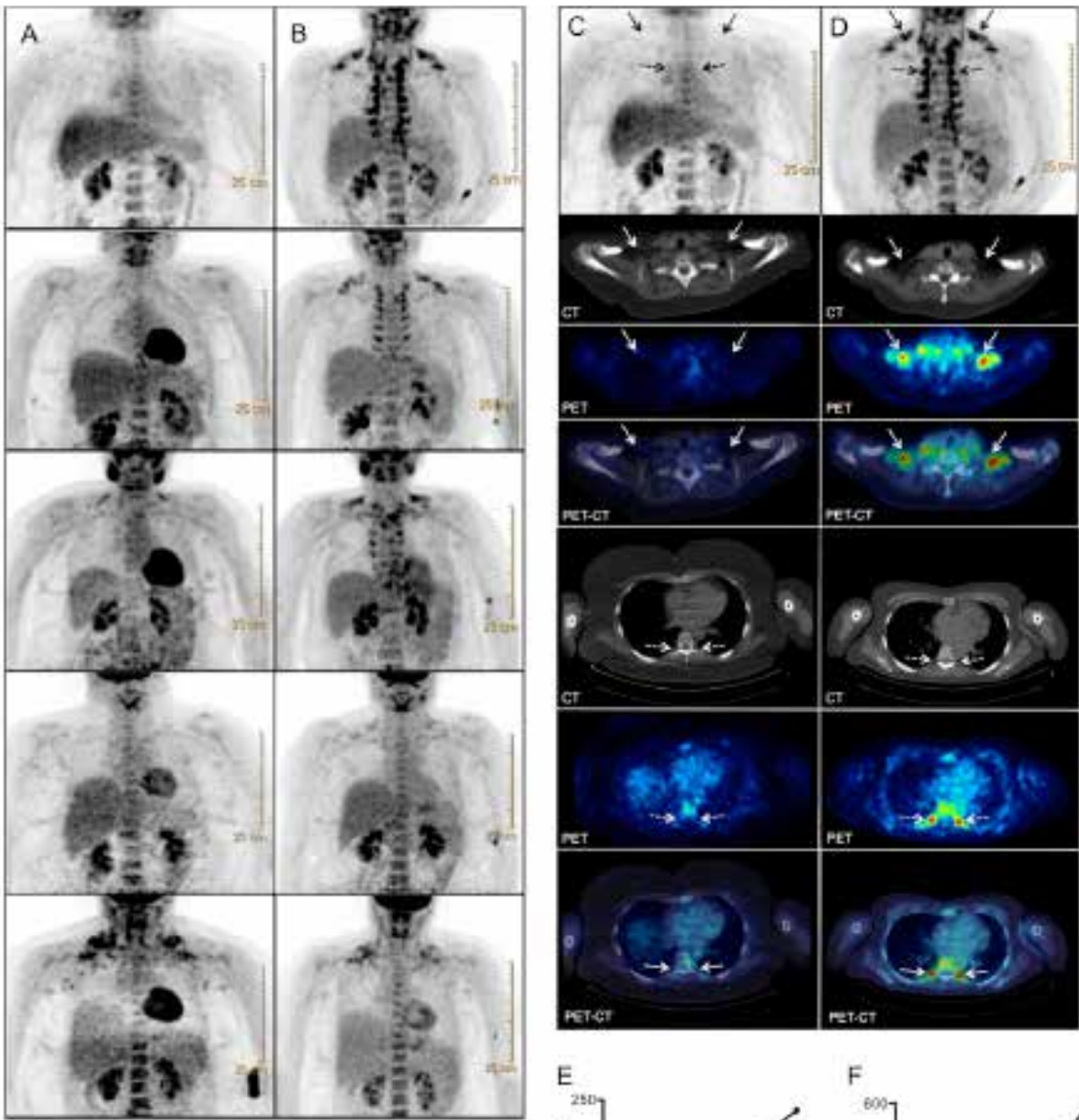

Rete
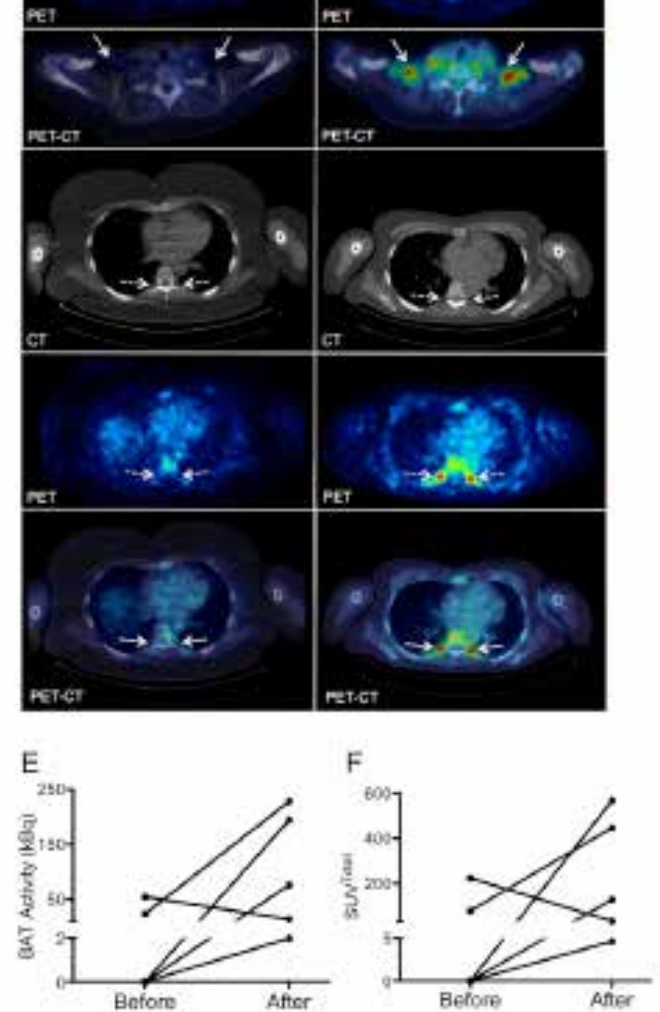

Figure 1. Brown adipose tissue (BAT) activity before and after weight loss. (A) PET-images of five morbidly obese subjects before bariatric surgery (A) and after weight loss (B). (C) PET-image, CT-image and PET-CTfusion-image of the subject that showed the largest increase in BAT activity before (C) and after weight loss (D). BAT activity was recruited in both supraclavicular and paravertebral areas, indicated by full and dashed arrows respectively. Full arrows indicate bilateral supraclavicular regions where BAT activity was observed after weight loss. Dashed arrows indicate bilateral paravertebral regions where BAT activity was observed after weight loss. (E) BAT activity in kiloBequerel $(\mathrm{kBq})$ and Total Standard Uptake Value (SUVTotal, F) before and after weight loss for the five subjects depicted in (A) and (B).

Abbreviations; CT: Computed Tomography, PET: Positron Emission Tomography. Baseline characteristics of subjects shown are from 10 . 

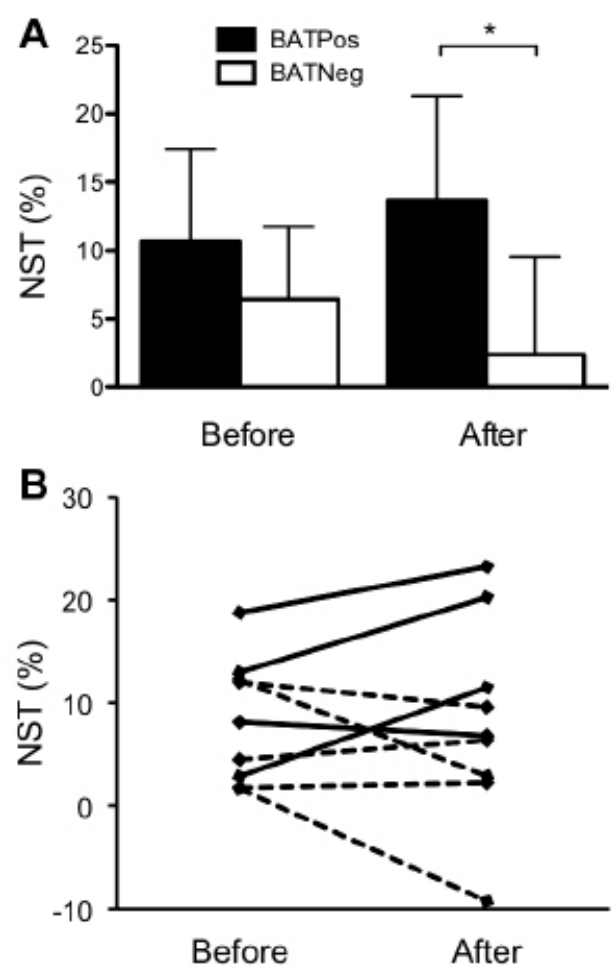

Figure 2. Relation between Brown Adipose Tissue (BAT) activity and Non-Shivering Thermogenesis (NST). (A) NST in BAT-positive (BATPos, n=5) and BAT15-negative (BATNeg, n=5) subjects before and after weight loss. (B) Non-shivering thermogenesis (NST) before and after weight loss. Full lines indicate subjects with BAT activity after weight loss (BAT-positive, $n=5$, one value not shown), dashed lines indicate subjects without BAT activity after weight loss (BAT-negative, $\mathrm{n}=5$ ). ${ }^{\star} \mathrm{P}<0.05$

(SUV, as calculated by uptake $(\mathrm{kBq} / \mathrm{mL})$ / injected dose $(\mathrm{kBq})$ / patient weight $(\mathrm{g})$ ). BAT activity of each region is determined by the average SUV uptake (SUVMean) times the volume of the region $\left(\mathrm{cm}^{3}\right)$, expressed as SUV'otal. Basal metabolic rate $(B M R)$ correlated significantly to fat-free mass (FFM) before and after weight loss (respectively $r=0.912, P=0.001$ and $r=0.948, P<0.001$ ). Therefore, we corrected BMR for FFM using linear regression. ${ }^{15}$ Percentage of weight loss is expressed as [(Beginning weight - Follow-up weight) / Beginning weight) $x$ 100]. Repeated measurements were compared using paired student's t-tests. Based on BAT presence after weight loss, subjects were categorized as BAT-positive or BAT-negative which was compared using unpaired student's t-tests.

\section{Results and discussion}

One year after LAGB mean body composition decreased significantly by $36.1 \pm 8.3$ 
$\mathrm{kg}$ with a mean percentage of weight loss of $28.65 \pm 6.23 \%$ (Table $1 \mathrm{~A}$ ). BAT activity was measured after applying an individualised cooling protocol. Thus, maximal BAT activity is achieved by low environmental temperatures just above the temperature that causes shivering. ${ }^{10}$ Mean room temperatures during maximal NST were significantly higher after weight loss (WL) (before WL: $14.2^{\circ} \mathrm{C}$ (range 12-17), after $W L: 15.7^{\circ} \mathrm{C}$ (range 12-21), $\mathrm{P}=0.026$ ). Before surgery, we observed - albeit minimal - cold induced BAT activity in supraclavicular depots in two female subjects. Their mean BAT activity $(39.0 \mathrm{kBq}$; range $23.6,54.5 \mathrm{kBq})$ and volume $\left(35.6 \mathrm{~cm}^{3}\right.$; range $22.5,48.6 \mathrm{~cm}^{3}$ ) were low (for group results see Table $1 \mathrm{~B}$, baseline characteristics based on ${ }^{10}$ ). After WL, we observed BAT in five out of ten subjects, among whom were the two above mentioned female subjects (Figure 1). Of these five BAT-positive subjects (all female, the two male subjects did not show BAT activity), one subject increased her BAT activity (23.6 kBq to $228.3 \mathrm{kBq}$ ), three previously BAT-negative subjects were BAT-positive after WL and one female subject decreased her BAT activity $(54.5 \mathrm{kBq}$ to $13.8 \mathrm{kBq}$ ). In addition to supraclavicular BAT activity, the depots of active BAT also included paravertebral depots in three patients (Figure 1). There were no significant differences in body composition between subjects characterized as BAT-positive and BAT-negative after WL, neither before nor after WL.

The basal metabolic rate (BMR) showed a significant decrease after WL $(95.3 \pm 4.5$ versus $88.8 \pm 4.8$ Watt, $P=0.028$ ). BAT-positive subjects (after $W L$ ) showed significantly higher NST compared to BAT-negative subjects $(13.7 \pm 7.7$ versus $2.4 \pm 7.1 \%, P=0.042$, Figure 2), in line with previous non-interventional studies on BAT and NST. ${ }^{10,11}$

We show that the presence and activity of BAT in adult humans is dynamic and can increase after weight reduction. This important finding shows for the first time using functional tools ( ${ }^{18} \mathrm{~F}-\mathrm{FDG}-\mathrm{PET}-\mathrm{CT}$ and indirect calorimetry) that BAT recruitment in humans is possible. Reduced activity of BAT could potentially impair total daily energy expenditure and be of importance in the development of obesity. LAGB induces a caloric restriction that caused significant WL. It does not seem likely LAGB could be responsible for other mechanisms that could have increased BAT, since the digestive tract is not altered. For example, gut hormones do not change after LAGB. ${ }^{16}$

The increase in BAT presence after WL could be a direct effect of a decreased insulation due to loss of subcutaneous adipose tissue. WL significantly changed the thermal distribution, as demonstrated by the observed skin and core temperatures 
Table 2. Core temperature (TCore), mean skin temperature (TSkin), proximal skin temperature (TProx) and distal skin temperature (TDist) during baseline measurements (Base) and cold exposure (Cold) before and after weight loss. The gradient between core and mean skin temperature is denoted as TCore-TSkin. $\Delta$ TCore and $\Delta$ TSkin denote differences between baseline measurements and cold exposure. Subjects are indicated as BAT-positive/BAT-negative based on their BAT-presence after weight loss. P-values shown are paired samples student's t-tests for group, five BAT-positive (BAT + ) and five BAT-negative (BAT-) subjects before and after weight loss. ${ }^{\star} \mathrm{P}<0.05,{ }^{*} \mathrm{P}<0.001$

\begin{tabular}{|c|c|c|c|c|c|c|c|c|c|c|}
\hline & & \multicolumn{3}{|c|}{ Before } & \multicolumn{3}{|c|}{ After } & \multicolumn{3}{|c|}{ P-value } \\
\hline & & Group & BAT+ & BAT- & Group & BAT+ & BAT- & Group & BAT+ & BAT- \\
\hline \multirow[t]{2}{*}{ TCore $\left({ }^{\circ} \mathrm{C}\right)$} & Base & $37.2 \pm 0.3$ & $37.3 \pm 0.3$ & $37.1 \pm 0.4$ & $37.0 \pm 0.3$ & $37.1 \pm 0.3$ & $36.9 \pm 0.3$ & 0.176 & 0.532 & 0.103 \\
\hline & Cold & $37.4 \pm 0.4$ & $37.5 \pm 0.3$ & $37.3 \pm 0.4$ & $37.1 \pm 0.3$ & $37.1 \pm 0.3$ & $37.0 \pm 0.4$ & $0.010 *$ & 0.142 & $<0.001 * *$ \\
\hline \multirow[t]{2}{*}{ TSkin $\left({ }^{\circ} \mathrm{C}\right)$} & Base & $31.6 \pm 0.6$ & $31.6 \pm 0.8$ & $31.6 \pm 0.5$ & $33.3 \pm 0.5$ & $33.1 \pm 0.4$ & $33.4 \pm 0.5$ & $<0.001^{* *}$ & $0.013 *$ & $<0.001^{* *}$ \\
\hline & Cold & $27.7 \pm 1.5$ & $27.6 \pm 2.0$ & $27.9 \pm 1.0$ & $29.6 \pm 1.5$ & $30.0 \pm 1.7$ & $29.2 \pm 1.3$ & $<0.001^{* *}<$ & $<0.001^{* *}$ & $0.044^{*}$ \\
\hline \multirow{2}{*}{$\begin{array}{l}\text { TCore- } \\
\text { TSkin }\end{array}$} & Base & $5.6 \pm 0.6$ & $5.6 \pm 0.7$ & $5.5 \pm 0.6$ & $3.8 \pm 0.7$ & $4.0 \pm 0.7$ & $3.6 \pm 0.7$ & $<0.001^{* *}$ & $0.011^{*}$ & $<0.001^{* *}$ \\
\hline & Cold & $9.7 \pm 1.7$ & $10.0 \pm 2.1$ & $9.5 \pm 1.4$ & $7.5 \pm 1.7$ & $7.1 \pm 1.9$ & $7.8 \pm 1.6$ & $<0.001^{* *}$ & $0.001^{*}$ & $0.026^{*}$ \\
\hline$\Delta \mathrm{TCore}$ & & $0.2 \pm 0.2$ & $0.3 \pm 0.1$ & $0.1 \pm 0.2$ & $0.01 \pm 0.1$ & $-.01 \pm 0.1$ & $0.05 \pm 0.1$ & $0.008^{*}$ & $0.008 *$ & 0.326 \\
\hline$\Delta$ TSkin & & $-3.9 \pm 1.3$ & $-4.1 \pm 1.7$ & $-3.8 \pm 0.9$ & $-3.7 \pm 1.5$ & $-3.1 \pm 1.6$ & $-4.2 \pm 1.2$ & 0.491 & $0.034^{*}$ & 0.401 \\
\hline \multirow[t]{2}{*}{$\operatorname{TProx}\left({ }^{\circ} \mathrm{C}\right)$} & Base & $31.6 \pm 1.2$ & $32.1 \pm 1.5$ & $31.0 \pm 0.5$ & $34.4 \pm 0.5$ & $34.3 \pm 0.6$ & $34.5 \pm 0.4$ & $0.001 * *$ & $0.011^{*}$ & $<0.001 * *$ \\
\hline & Cold & $28.3 \pm 2.4$ & $28.8 \pm 3.4$ & $27.8 \pm 1.1$ & $31.0 \pm 2.0$ & $31.4 \pm 2.6$ & $30.5 \pm 1.4$ & $<0.001 * *$ & $0.006^{*}$ & $0.027^{*}$ \\
\hline \multirow[t]{2}{*}{ TDist $\left({ }^{\circ} \mathrm{C}\right)$} & Base & $31.8 \pm 1.1$ & $31.3 \pm 0.8$ & $32.2 \pm 1.2$ & $30.4 \pm 2.2$ & $29.5 \pm 1.5$ & $31.2 \pm 2.6$ & 0.085 & $0.042^{*}$ & 0.517 \\
\hline & Cold & $25.2 \pm 2.2$ & $24.6 \pm 2.7$ & $25.9 \pm 1.6$ & $24.6 \pm 2.3$ & $24.3 \pm 2.6$ & $24.9 \pm 2.1$ & 0.187 & 0.600 & 0.265 \\
\hline
\end{tabular}

(Table 2). Obese subjects show a low gradient between proximal and distal skin temperature during cold exposure, indicating a low level of vasoconstriction..$^{15}$ This gradient increased after $\mathrm{WL}$ (baseline; $0.2 \pm 1.9^{\circ} \mathrm{C}$ before $\mathrm{WL}$ and $-4.0 \pm 2.4^{\circ} \mathrm{C}$ after $\mathrm{WL}, \mathrm{P}=0.001$, cold exposure; $-3.1 \pm 1.9^{\circ} \mathrm{C}$ before $\mathrm{WL}$ and $-6.4 \pm 2.2^{\circ} \mathrm{C}$ after $W L, P=0.001)$, suggesting increased skin vasoconstriction. Insulation of the body's core, as measured by the gradient between core and mean skin temperature, decreased significantly after $W L$ (before $W L ; 5.6 \pm 0.6^{\circ} \mathrm{C}$, after $\mathrm{WL} ; 3.8 \pm 0.7^{\circ} \mathrm{C}$, $\mathrm{P}<0.001)$. This indicates the insulation of the body's core decreased, despite increased vasoconstriction of the skin. Compared to BAT-negative subjects, BAT-positive subjects showed a significantly smaller increase in core temperature 
( $\triangle \mathrm{TC}$ Core) during cold exposure, accompanied by a smaller decrease in mean skin temperature ( $\triangle$ TSkin) (Table 2 ), suggesting less vasoconstriction. ${ }^{15}$ Hence, increased thermogenesis in BAT could be responsible for this difference.

It should be noted that the BAT activity we observed in this study is low in comparison to the levels observed in lean young men $(51.5 \pm 87.8 \mathrm{kBq}$ versus $\left.428 \pm 394 \mathrm{kBq}, \mathrm{P}=0.008,{ }^{2}\right)$. BAT activity is suggested to be lower in older subjects, ${ }^{3}$ which could possibly affect the energy balance. Therefore, further studies are needed to investigate the impact of BAT recruitment at this level for the long-term treatment of obesity. Nevertheless, this study provides the first evidence that BAT can be recruited in adults. 


\section{References}

1. Nedergaard J, Bengtsson T, Cannon B. Unexpected evidence for active brown adipose tissue in adult humans. Am J Physiol Endocrinol Metab 2007;293:E444-52.

2. van Marken Lichtenbelt WD, Vanhommerig JW, Smulders NM, et al. Cold-activated brown adipose tissue in healthy men. N Engl J Med 2009;360:15008.

3. Saito $M$, Okamatsu-Ogura $Y$, Matsushita $M$, et al. High Incidence of Metabolically Active Brown Adipose Tissue in Healthy Adult Humans: Effects of Cold Exposure and Adiposity. Diabetes 2009;58:1526-31.

4. Virtanen KA, Lidell ME, Orava J, et al. Functional brown adipose tissue in healthy adults. N Engl J Med 2009;360:1518-25.

5. Isacsson A, Frederiksen SG, Nilsson P, Hedenbro JL. Quality of life after gastroplasty is normal: a controlled study. Eur J Surg 1997;163:181-6.

6. Cannon B, Nedergaard J. Brown adipose tissue: function and physiological significance. Physiol Rev 2004;84:277-359.

7. Petrovic N, Walden TB, Shabalina IG, Timmons JA, Cannon B, Nedergaard J. Chronic peroxisome proliferator-activated receptor gamma (PPARgamma) activation of epididymally derived white adipocyte cultures reveals a population of thermogenically competent, UCP1-containing adipocytes molecularly distinct from classic brown adipocytes. J Biol Chem 2009;285:7153-64.

8. Schulz TJ, Huang $\mathrm{TL}$, Tran TT, et al. Identification of inducible brown adipocyte progenitors residing in skeletal muscle and white fat. Proc Natl Acad Sci U S A 2011;108:143-8.

9. van Marken Lichtenbelt WD, Schrauwen P. Implications of non-shivering thermogenesis for energy balance regulation in humans. Am J Physiol Regul Integr Comp Physiol 2011.

10. Vijgen GH, Bouvy ND, Teule GJ, Brans B, Schrauwen P, van Marken Lichtenbelt WD. Brown adipose tissue in morbidly obese subjects. Plos One 2011;6:e17247.

11. Yoneshiro T, Aita S, Matsushita M, et al. Brown Adipose Tissue, WholeBody Energy Expenditure, and Thermogenesis in Healthy Adult Men. Obesity (Silver Spring) 2011;19:13-6.

12. Bligh J, Johnson KG. Glossary of terms for thermal physiology. J Appl Physiol 1973;35:941-61.

13. van Marken Lichtenbelt WD, Daanen HA, Wouters $L$, et al. Evaluation 
of wireless determination of skin temperature using iButtons. Physiol Behav 2006;88:489-97.

14. Kingma $B$, Frijns $A$, van Marken Lichtenbelt W. The thermoneutral zone: implications for metabolic studies. Front Biosci (Elite Ed) 2012;4:1975-85.

15. Wijers SL, Saris WH, van Marken Lichtenbelt WD. Cold-induced adaptive thermogenesis in lean and obese. Obesity (Silver Spring) 2010;18:1092-9.

16. Bose $M$, Machineni S, Olivan B, et al. Superior appetite hormone profile after equivalent weight loss by gastric bypass compared to gastric banding. Obesity (Silver Spring) 2010;18:1085-91. 


$$
\theta_{-1}
$$




\section{Chapter 5}

\section{Increased skeletal muscle mitochondrial function in morbidly obese subjects one year after bariatric surgery}




\section{Abstract}

\section{Background}

Obesity and type 2 diabetes are associated with impaired skeletal muscle mitochondrial metabolism. As an intrinsic characteristic of an individual, skeletal muscle mitochondrial dysfunction could be a risk factor for weight gain and obesityassociated comorbidities as type 2 diabetes. On the other hand, impaired skeletal muscle metabolism could be a consequence of obesity. We hypothesize that marked weight loss after bariatric surgery increases skeletal muscle mitochondrial function in formerly morbidly obese subjects.

\section{Methods}

Skeletal muscle mitochondrial function as assessed by high-resolution respirometry was determined in eight morbidly obese subjects (BMl; $41.3 \pm 4.7 \mathrm{~kg} / \mathrm{m}^{2}$, Body Fat; $48.3 \pm 5.2 \%$ ) before and one year after bariatric surgery (mean weight loss: $35.0 \pm 8.6 \mathrm{~kg})$.

\section{Results}

ADP-stimulated respiration significantly increased after weight loss upon mitochondrial complex I-linked substrates (for malate+glutamate; $4.23 \pm 1.38$ versus $6.79 \pm 2.03 \mathrm{O}_{2}$-flux, $\mathrm{P}=0.029$; for malate+octanoyl-carnitine+glutamate (MOG); $4.19 \pm 2.12$ versus $8.93 \pm 4.16 \mathrm{O}_{2}$-flux, $\mathrm{P}=0.028$ ) as well as upon parallel electron input into both complex I and II (for malate+octanoyl-carnitine+glutamate+succinate (MOGS); $10.02 \pm 3.23$ versus $13.97 \pm 6.63 \mathrm{O}_{2}$-flux, $\mathrm{P}=0.031$ ).

\section{Conclusions}

We conclude that low skeletal muscle mitochondrial function is a consequence of obesity that significantly improves after marked weight loss induced by bariatric surgery. The pronounced amount of weight loss associated with bariatric surgery appears to be crucial since previous studies with limited weight loss remained without effect on skeletal muscle mitochondrial function. Future molecular studies should investigate the processes that are related to the observed change in skeletal muscle mitochondrial function upon bariatric surgery. 


\section{Introduction}

Obesity is one of the Western world's primary health care issues and the increasing incidence of obesity is accompanied by an equal rise in comorbidities as type 2 diabetes, dyslipidemia and hepatosteatosis. ${ }^{1}$ The excess amount of adipose tissue in obesity is associated with a dysfunction in several tissues; hepatosteatosis, which causes impaired liver function and increased lipid deposition in skeletal muscle is suggested to induce insulin resistance., ${ }^{1,2}$ In skeletal muscle from obese and obese type 2 diabetic subjects, a decreased mitochondrial function compared to lean and nondiabetic subjects has been reported. This could exacerbate the negative effect of IMCL accumulation on insulin sensitivity. ${ }^{3-7}$ However, although diet-induced weight loss decreased intramyocellular lipid content and has been shown to improve skeletal muscle insulin sensitivity in nondiabetic overweight subjects, ${ }^{8,9}$ skeletal muscle mitochondrial function (as determined by NADH-oxidase enzyme levels) per se did not change..$^{8,10}$ This may suggest that reduced mitochondrial function in obesity is an intrinsic characteristic of obese subjects. However, it should be noted that weight loss in the above-mentioned studies was low $(9-18 \mathrm{~kg})^{8-10}$ in comparison to bariatric surgery $(37-42 \mathrm{~kg})^{11}$ and it cannot be excluded that further weight reduction does improve skeletal muscle mitochondrial metabolism. Indeed, it has been shown before that proteins associated with mitochondrial biogenesis in skeletal muscle were enhanced and related to improved insulin sensitivity after bariatric surgery, suggesting that skeletal muscle mitochondrial function may be suppressed by excessive body weight.12,13 However, to our knowledge no study has evaluated the effect of bariatric surgery on skeletal muscle mitochondrial function per se and therefore these findings need further confirmation. To test our hypothesis that skeletal muscle mitochondrial function improves after bariatric surgery we investigated ex-vivo skeletal muscle mitochondrial function in morbidly obese subjects before and one year after laparoscopic adjustable gastric banding (LAGB) surgery.

\section{Methods}

\section{Study protocol}

Approval was obtained from the Maastricht University Medical Centre institutional review board. Written informed consent was received from two male and six female morbidly obese subjects who were awaiting laparoscopic adjustable gastric banding 
(LAGB), with a mean BMI of $41.3 \pm 4.7 \mathrm{~kg} / \mathrm{m}^{2}$ (Table 1). One female subject used levothyroxine for hypothyroidism and was euthyroid for several years. Diabetes mellitus was an exclusion criterion.

\section{Body composition}

Body composition (Fat Free Mass, Fat Mass) was determined under fasted conditions by dual x-ray absorptiometry (DXA, type Discovery A, Hologic, Bedford, USA).

\section{Muscle biopsies}

Several weeks before surgery, a muscle biopsy according to Bergström et al. ${ }^{14}$ was taken under fasted conditions at $\sim 8: 30$ in the morning. Part of the muscle was directly used for the fresh preparation of permeabilized muscle fibers as described. ${ }^{3}$ The remaining part of the muscle was directly frozen in liquid nitrogen and stored at $-80^{\circ} \mathrm{C}$ until further analysis.

To determine skeletal muscle mitochondrial capacity, high-resolution respirometry was performed in permeabilized muscle fibers at $37^{\circ} \mathrm{C}$ in a two-chamber-Oxygraph (OROBOROS $^{\circledR}$ Instruments, Innsbruck, Austria), essentially according to Hoeks et al. ${ }^{15}$ Coupled (state 3 ) respiration, initiated by addition of $2 \mathrm{mM} \mathrm{ADP}$, was measured for complex I-linked substrate combination malate+glutamate, both in the presence and absence of the lipid substrate octanoyl-carnitine. Coupled respiration was then maximized with convergent electron input through complex I and complex II, by adding saturating concentrations of succinate $(10 \mathrm{mM})$. Subsequently, mitochondrial respiration uncoupled from ATP synthesis (state 4) was determined via addition of the ATP-synthase inhibitor oligomycin. In addition, the chemical uncoupler FCCP (carbonyl-cyanide-4-(trifluoromethoxy)-phenylhydrazone) was used to maximize oxygen flux to assess maximal mitochondrial capacity (state u respiration).

\section{Normalisation for mitochondrial DNA content}

To determine mitochondrial density, the mitochondrial DNA (mtDNA) copy numbers were determined according to Phielix et al., ${ }^{3}$ and all muscle biopsy samples were run in the same analysis. For comparison, oxygen fluxes per wet-weight muscle mass were normalized for mtDNA. The mitochondrial DNA copy numbers did not differ significantly before and after weight loss (mtDNA copy number; $960.6 \pm 425.1$ before, $868.8 \pm 386.0$ after weight loss, $P=0.371$ ). All results showed similar trends 
and significance when oxygen fluxes were not corrected for mtDNA (results not shown). Oxygen fluxes $\left(\mathrm{O}_{2}\right.$ flux $)$ shown are expressed as picomoles $\mathrm{O}_{2} \times$ seconds $^{-1}$ $x$ milligrams of tissue ${ }^{-1} \times$ mtDNA $\times 10^{6}$.

\section{Plasma values}

Plasma TSH and FT4 were measured using an electrochemiluminescence immunoassay (Roche, Basel, Switzerland) and a fluoroimmunoassay (PerkinElmer, Massachusetts, USA). Plasma insulin, glucose, FFA and the HOMA-IR were calculated as described. ${ }^{16}$

\section{Follow-up}

All eight subjects underwent LAGB without any perioperative or postoperative complications. One year after LAGB a second muscle biopsy was taken.

\section{Statistical analysis}

Reported data were expressed as means \pm SD. Statistical analyses were performed with PASW Statistics 18.0 for Mac OS X 10.6.4. Repeated measurements before and one year after bariatric surgery were compared using paired student's t-tests. A P-value below 0.05 was considered to be significant.

\section{Results and discussion}

Body composition was significantly altered one year after surgery (Table 1) with a mean weight loss of $27.9 \pm 6.6 \%$. After a mean follow-up of 18 months, there was no difference in body composition compared to the one-year time point (body mass; $91.7 \pm 18.8$ versus $91.5 \pm 18.3 \mathrm{~kg}$, BMl; $29.8 \pm 4.8$ versus $29.7 \pm 4.7 \mathrm{~kg}$ / $\mathrm{m}^{2}, \mathrm{P}=0.844$ ), which indicates patients were weight stable at the moment of the second muscle biopsy.

ADP-stimulated (state 3 ) respiration increased after weight loss upon complex I-linked substrates (for malate+glutamate; $4.23 \pm 1.38$ versus $6.79 \pm 2.03 \mathrm{O}_{2}$ flux, $\mathrm{P}=0.029$; for malate+octanoyl-carnitine+glutamate (MOG); $4.19 \pm 2.12$ versus $8.93 \pm 4.16 \mathrm{O}_{2}$ flux, $\mathrm{P}=0.028$ ) as well as upon parallel electron input into both complex I and complex II (for malate+octanoyl-carnitine+glutamate+succinate (MOGS); 10.02 \pm 3.23 versus $13.97 \pm 6.63 \mathrm{O}_{2}$ flux, $\mathrm{P}=0.031$ ), although did this not reach statistical significance for malate+glutamate+succinate (MGS; $9.50 \pm 2.43$ versus $12.31 \pm 5.50 \mathrm{O}_{2}$ flux, $\mathrm{P}=0.086$, Figure 1). Leak respiration upon the ATP-synthase inhibitor oligomycin, 
i.e. respiration not related to ATP synthesis (state 4 respiration), was determined as a marker for mitochondrial uncoupling, but remained unaffected by weight loss $\left(6.13 \pm 2.66\right.$ versus $5.42 \pm 2.37 \mathrm{O}_{2}$ flux, $\mathrm{P}=0.093$, Figure 1$)$. The relative contribution of state 4 to state 3 respiration (uncoupling ratio; UCR) decreased significantly after weight loss $(0.63 \pm 0.19$ versus $0.45 \pm 0.07, P=0.049$, Figure 1$)$. Maximally uncoupled mitochondrial respiration, as analysed after addition of FCCP in the presence of octanoyl-carnitine, did not increase significantly after weight loss $(14.9 \pm 4.45$ versus $18.04 \pm 8.18 \mathrm{O}_{2}$ flux, $\mathrm{P}=0.212$, Figure 1).

In this study we investigated the effect of pronounced weight loss induced by bariatric surgery on skeletal muscle mitochondrial function in morbidly obese subjects. ADP-stimulated (state 3 ) respiration increased significantly one year after bariatric surgery. Our data suggest that low skeletal muscle mitochondrial function in morbidly obese subjects is reversible by bariatric surgery-induced weight loss.

Skeletal muscle fat oxidation is negatively related to $\mathrm{BMI},{ }^{17}$ resulting in an increased muscle fat accumulation in obesity. ${ }^{9,18}$ This impaired lipid oxidation is associated with skeletal muscle insulin resistance and thus suggests a pathophysiological basis for obesity-related type 2 diabetes. ${ }^{9}$ In line with this notion, skeletal muscle mitochondria from obese type 2 diabetics displayed a reduced

activity of the mitochondrial oxidative enzyme NADH-reductase, suggesting an impairment of mitochondrial function, ${ }^{5}$ which was confirmed by in vivo measurements. ${ }^{19}$ Furthermore, a detailed ex vivo analysis of mitochondrial function in permeabilized skeletal muscle fibres showed that the observed in vivo mitochondrial dysfunction in overweight type 2 diabetes (mean BMI; 28.9 $\mathrm{kg} / \mathrm{m}^{2}$ ) was associated with a decreased intrinsic mitochondrial capacity, i.e. ADP-stimulated (state 3 ) mitochondrial respiration corrected for mitochondrial density. ${ }^{3}$ Skeletal muscle mitochondrial state 3 respiration was also shown to be reduced in non-diabetic morbidly obese subjects eligible for bariatric surgery (mean $\mathrm{BMI} ; 40 \mathrm{~kg} / \mathrm{m}^{2}$ ) compared to lean controls. ${ }^{6}$ To observe whether obesity is causative for a decreased mitochondrial function, several reports have studied the effect of weight loss on skeletal muscle mitochondrial function, in both type 2 diabetic and nondiabetics. A diet-induced weight loss of $10.6 \mathrm{~kg}$ did not induce changes in mitochondrial NADH-reductase activity in nondiabetic obese subjects (mean BMI before weight loss; $\left.33.4 \mathrm{~kg} / \mathrm{m}^{2}\right)^{8}$ and one report even observed a decrease in mitochondrial cytochrome c oxidase activity after weight loss (12-17 kg weight 
Table 1. Subject characteristics for two male and six female morbidly obese subjects before and after weight loss. ${ }^{\star} \mathrm{P}<0.05,{ }^{*} \mathrm{P}<0.001$. BMI denotes Body Mass Index.

\begin{tabular}{lccc} 
Characteristics & Before & After & P-value \\
\hline Age (years) & $40 \pm 9$ & $41 \pm 9$ & $<0.001^{* *}$ \\
Body mass $(\mathrm{kg})$ & $126.71 \pm 19.98$ & $91.71 \pm 18.81$ & $<0.001^{* *}$ \\
BMI $\left(\mathrm{kg} / \mathrm{m}^{2}\right)$ & $41.28 \pm 4.75$ & $29.82 \pm 4.78$ & $<0.001^{* *}$ \\
Body Fat \% (BF\%) & $48.30 \pm 5.21$ & $34.11 \pm 8.47$ & $<0.001^{* *}$ \\
Fat Mass $(\mathrm{kg})$ & $61.45 \pm 11.47$ & $32.15 \pm 11.79$ & $<0.001^{* *}$ \\
Fat Free Mass $(\mathrm{kg})$ & $63.79 \pm 13.02$ & $58.98 \pm 13.18$ & $<0.001^{* *}$
\end{tabular}

loss, initial $\left.\mathrm{BMl} ; 33-34 \mathrm{~kg} / \mathrm{m}^{2}\right) .^{10}$ In contrast, after a more pronounced diet-induced weight loss in nondiabetics (13-32 kg weight loss, initial $\left.\mathrm{BMl} ; 33.8 \mathrm{~kg} / \mathrm{m}^{2}\right)$, an improved oxidative enzyme (succinate dehydrogenase) activity was observed. ${ }^{20}$ Bariatric surgery results in pronounced weight loss and after Roux-en-Y-gastricbypass and bilio-pancreatic-diversion surgery (42-63 kg weight loss, mean initial $\mathrm{BMl} ; 45.9-53.5 \mathrm{~kg} / \mathrm{m}^{2}$ ) proteins associated with mitochondrial biogenesis (PGC1a and MFN2) were enhanced in skeletal muscle and related to improved insulin sensitivity as assessed by euglycemic-hyperinsulinaemic clamp. ${ }^{12,13}$ Finally, in subjects with a $\sim 50 \mathrm{~kg}$ weight loss after Roux-en-Y-gastric-bypass, skeletal muscle fatty acid oxidation (as assessed ex vivo with ${ }^{14} \mathrm{C}$-palmitate) did not change and was decreased compared to lean controls. ${ }^{21}$ However, the postoperative BMI was relatively high $\left(36.5 \mathrm{~kg} / \mathrm{m}^{2}\right)$ compared to the current study $\left(30 \mathrm{~kg} / \mathrm{m}^{2}\right)$.

In contrast to the abovementioned weight loss studies using biochemical assessments of mitochondrial enzyme activities to assess skeletal muscle mitochondrial function we here performed a detailed characterisation of mitochondrial function via ex vivo mitochondrial respiration in permeabilized muscle fibers. In this study we show that ADP-stimulated state 3 respiration increased after a mean $35-\mathrm{kg}$ weight loss. The mitochondrial density (as assessed by mtDNA copy numbers) did not change after weight loss and therefore the observed improvement in mitochondrial respiration is an intrinsic mitochondrial characteristic. The improvement in muscle mitochondrial metabolism suggests that the observed weight loss could 'restore' obesity-induced impairments in skeletal muscle mitochondrial function. Further studies should elucidate the molecular and clinical changes associated with the increase skeletal muscle mitochondrial function. All included morbidly obese subjects were non-diabetic and plasma values (Table 2) for plasma insulin showed a nearly significant decrease $(P=0.064)$. However, the homeostasis model for insulin resistance (HOMA-IR) did not change after weight loss $(P=0.324)$. Although fasting insulin levels tended to be lower after weight loss (mean reduction; $28.6 \%$ ) 
A

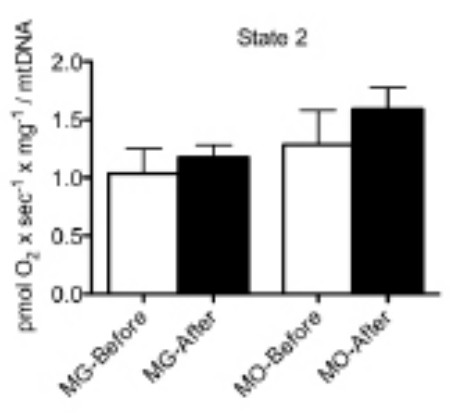

C

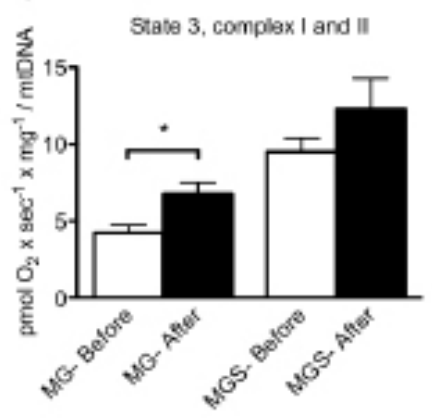

E

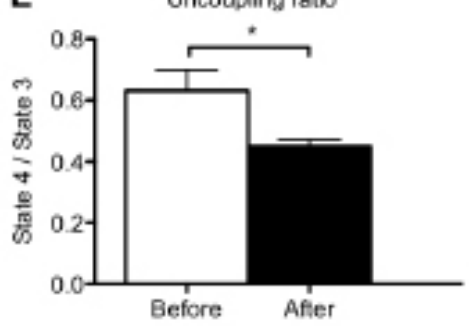

B

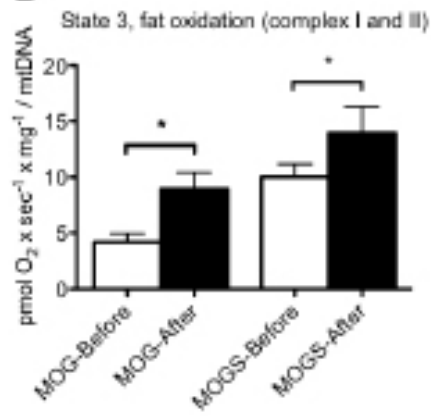

D
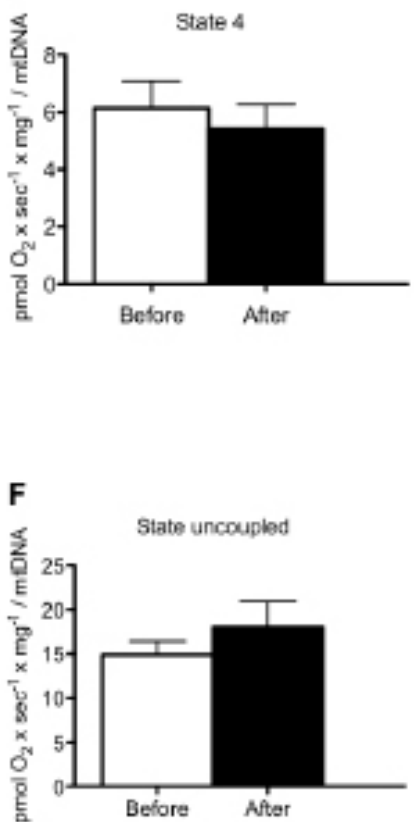

Figure 1. Skeletal muscle fibre respirometry before (white bars) and after weight loss (black bars). A: Oxygen consumption in the presence of endogenous substrates (state 2 respiration) was similar before and after weight loss $(\mathrm{P}=0.310)$. B: ADP stimulated state 3 respiration upon a lipid substrate for complex I (malate, octanoyl-carnitine and glutamate (MOG) and complex I\&II substrates (malate, octanoyl-carnitine, glutamate and succinate (MOGS)). C: State 3 respiration upon complex I substrates (malate, glutamate (MG)) and complex I\&II substrates (malate, glutamate and succinate (MGS)). D: Leak (state 4) respiration obtained by addition of the ATP synthase inhibitor oligomycin. E: The relative contribution of state 4 to state 3 respiration (uncoupling ratio; UCR). F: Maximal respiration upon FCCP (state uncoupled). O2 flux shown: picomoles $\mathrm{O} 2$ consumption $\mathrm{x}$ seconds- $1 \mathrm{x}$ milligrams of tissue- $1 \mathrm{x} \mathrm{mtDNA} x \mathrm{106}$. Values shown are mean+S.E.M., $\mathrm{n}=8 .{ }^{*} \mathrm{P}<0.05$ 
Table 2. Plasma values before and after weight loss. Values shown for two male and six female morbidly obese subjects before and after weight loss. ${ }^{\star} \mathrm{P}<0.05,{ }^{*} \mathrm{P}<0.001$. TSH denotes Thyroid-Stimulating Hormone, FT4; Free Thyroxine, HOMA-IR; Homeostasis Model Assessment for Insulin Resistance, FFA; Free Fatty Acids.

\begin{tabular}{llll} 
& Before & After & P-value \\
\hline TSH $(\mathrm{mU} / \mathrm{mL})$ & $2.03 \pm 0.7$ & $1.85 \pm 0.4$ & 0.341 \\
FT4 $(\mathrm{pmol} / \mathrm{L})$ & $13.53 \pm 1.94$ & $14.85 \pm 1.43$ & $0.009^{*}$ \\
Glucose $(\mathrm{mM})$ & $4.86 \pm 0.38$ & $5.22 \pm 1.04$ & 0.199 \\
Insulin $(\mu \mathrm{U} / \mathrm{mL})$ & $25.44 \pm 8.52$ & $16.64 \pm 5.16$ & 0.064 \\
HOMA-IR & $4.90 \pm 2.87$ & $3.80 \pm 1.13$ & 0.324 \\
FFA $(\mu \mathrm{M})$ & $588.34 \pm 234.34$ & $567.0 \pm 283.43$ & 0.750
\end{tabular}

further studies in morbidly obese diabetic populations are necessary to determine the effect of weight loss on skeletal muscle mitochondrial function and its relation to obesity-induced insulin resistance.

In summary, this study shows an increase in ex vivo skeletal muscle mitochondrial function in morbidly obese subjects one year after LAGB-induced weight loss. The level of weight loss in this study was higher than previous reports that did not observe differences in mitochondrial function after weight loss. Possibly, the very pronounced weight loss after bariatric surgery is necessary to recover mitochondrial function in morbid obesity. 


\section{References}

1. Haslam DW, James WP. Obesity. Lancet 2005;366:1197-209.

2. Timmers S, Schrauwen $P$, de Vogel J. Muscular diacylglycerol metabolism and insulin resistance. Physiol Behav 2008;94:242-51.

3. Phielix $E$, Schrauwen-Hinderling $V B$, Mensink $M$, et al. Lower intrinsic ADP-stimulated mitochondrial respiration underlies in vivo mitochondrial dysfunction in muscle of male type 2 diabetic patients. Diabetes 2008;57:2943-9. 4. Boyle KE, Zheng D, Anderson EJ, Neufer PD, Houmard JA. Mitochondrial lipid oxidation is impaired in cultured myotubes from obese humans. Int $J$ Obes (Lond) 2011.

5. Kelley DE, He J, Menshikova EV, Ritov VB. Dysfunction of mitochondria in human skeletal muscle in type 2 diabetes. Diabetes 2002;51:2944-50.

6. Bakkman L, Fernstrom M, Loogna P, Rooyackers O, Brandt L, Lagerros YT. Reduced respiratory capacity in muscle mitochondria of obese subjects. Obes Facts 2010;3:371-5.

7. Schrauwen $P$, Schrauwen-Hinderling V, Hoeks J, Hesselink MK. Mitochondrial dysfunction and lipotoxicity. Biochim Biophys Acta 2010;1801:26671.

8. Toledo FG, Menshikova EV, Azuma K, et al. Mitochondrial capacity in skeletal muscle is not stimulated by weight loss despite increases in insulin action and decreases in intramyocellular lipid content. Diabetes 2008;57:987-94.

9. Kelley DE, Goodpaster B, Wing RR, Simoneau JA. Skeletal muscle fatty acid metabolism in association with insulin resistance, obesity, and weight loss. Am J Physiol 1999;277:E1130-41.

10. Simoneau JA, Veerkamp JH, Turcotte LP, Kelley DE. Markers of capacity to utilize fatty acids in human skeletal muscle: relation to insulin resistance and obesity and effects of weight loss. FASEB J 1999;13:2051-60.

11. Buchwald $H$, Avidor $Y$, Braunwald $E$, et al. Bariatric surgery: a systematic review and meta-analysis. JAMA 2004;292:1724-37.

12. Gastaldi G, Russell A, Golay A, et al. Upregulation of peroxisome proliferator-activated receptor gamma coactivator gene (PGC1A) during weight loss is related to insulin sensitivity but not to energy expenditure. Diabetologia 2007; 50:2348-55.

13. Hernandez-Alvarez MI, Chiellini $C$, Manco $M$, et al. Genes involved in mitochondrial biogenesis/function are induced in response to bilio-pancreatic diversion in morbidly obese individuals with normal glucose tolerance but not in 
type 2 diabetic patients. Diabetologia 2009;52:1618-27.

14. Bergstrom J, Hermansen L, Hultman E, Saltin B. Diet, muscle glycogen and physical performance. Acta Physiol Scand 1967;71:140-50.

15. Hoeks J, van Herpen NA, Mensink $M$, et al. Prolonged fasting identifies skeletal muscle mitochondrial dysfunction as consequence rather than cause of human insulin resistance. Diabetes 2010;59:2117-25.

16. Wijers SL, Saris WH, van Marken Lichtenbelt WD. Cold-induced adaptive thermogenesis in lean and obese. Obesity (Silver Spring) 2010;18:1092-9.

17. Kim JY, Hickner RC, Cortright RL, Dohm GL, Houmard JA. Lipid oxidation is reduced in obese human skeletal muscle. Am J Physiol Endocrinol Metab 2000;279:E1039-44.

18. Boyle KE, Canham JP, Consitt LA, et al. A high-fat diet elicits differential responses in genes coordinating oxidative metabolism in skeletal muscle of lean and obese individuals. J Clin Endocrinol Metab 2011;96:775-81.

19. Schrauwen-Hinderling VB, Kooi ME, Hesselink $M K$, et al. Impaired in vivo mitochondrial function but similar intramyocellular lipid content in patients with type 2 diabetes mellitus and BMI-matched control subjects. Diabetologia 2007;50:113-20.

20. Kern PA, Simsolo RB, Fournier M. Effect of weight loss on muscle fiber type, fiber size, capillarity, and succinate dehydrogenase activity in humans. J Clin Endocrinol Metab 1999;84:4185-90.

21. Berggren JR, Boyle KE, Chapman WH, Houmard JA. Skeletal muscle lipid oxidation and obesity: influence of weight loss and exercise. Am J Physiol Endocrinol Metab 2008;294:E726-32. 


$$
\theta_{-1}
$$




\section{Chapter 6}

\section{Increased oxygen consumption in human adipose tissue from the "brown adipose tissue" region}




\section{Abstract}

\section{Background}

Since the discovery of functional 'brown adipose tissue (BAT)' in adult man, there has been a renewed interest in the physiology of human BAT. Imaging studies using FDG-PET-CT have shown increased glucose uptake in adipose tissue regions assumed to be BAT in humans. However, so far this adipose tissue depot has not been characterized with respect to cellular and mitochondrial capacity. We here determine basal cellular oxygen consumption in human adipose tissue (AT) obtained from biopsies taken from the neck region, known to be FDG-PET-CTpositive in previous studies.

\section{Methods}

Mitochondrial respirometry in AT from the human BAT region was compared to the cellular respiration in subcutaneous white adipose tissue (WAT) taken from the neck of the same subject.

\section{Results}

Adipose tissue from the human BAT region displayed increased cellular mitochondrial respiration, on average three times higher compared to subcutaneous WAT. The difference in basal respiration was much more pronounced than the previous reported $10-20 \%$ higher mitochondrial respiration in visceral adipose tissue, and illustrates that adipose tissue from human BAT region is a distinct adipose tissue depot with high mitochondrial capacity, similar to studies performed in rodents. Presence of uncoupling protein 1 (UCP-1) was confirmed by immunostaining as performed before.

\section{Conclusions}

The results of this study suggest that human adipose tissue, taken from the region that was shown to be BAT-positive, can indeed be distinguished from subcutaneous WAT by the presence of UCP-1 and a significantly higher basal mitochondrial activity. Future studies should be directed to the further metabolic and bioenergetic characterization of this adipose tissue depot. 


\section{Introduction}

Since the discovery of functional brown adipose tissue (BAT) in adult man, ${ }^{1-3}$ there has been a vast increase in the scientific interest in the physiology of human BAT. PET-CT-studies (Positron-Emission-Tomography-and-Computed-Tomography) using the glucose tracer ${ }^{18} \mathrm{~F}$-FDG (Fluorine-18-Fluoro-Deoxy-Glucose) have shown that cold-induced BAT activity is inversely related to body fat percentage and subjects with glucose uptake in BAT have higher thermogenesis. ${ }^{2,4,5}$ In addition, other studies have now indirectly measured in vivo BAT activity by means of different metabolic tracers. ${ }^{6,7}$ Collectively, these studies show increased metabolic activity in an anatomical region presumed to contain BAT in humans, located in the adipose tissue depot dorsal from the neck muscles. To date, adipose tissue in this region has not been characterized in great detail ex vivo, although some, mainly smaller studies have shown the presence of UCP1 and thermogenic preadipocytes in this adipose tissue. ${ }^{3,6,8}$ Very recently, we showed that adipocytes from this region can in fact be classified as beige fat cells; adipocytes genetically distinct from white and brown adipose tissue, but with the capacity to induce high levels of UCP1 expression as in BAT. ${ }^{9}$

Here we had the unique opportunity to further characterize perioperative neck adipose tissue (AT) biopsies taken from the area that is now recognized as human "BAT" based on FDG-PET-CT positive glucose uptake and determined cellular respirometry in comparison to subcutaneous WAT from the neck from the same subject. We aimed to investigate if the AT from the BAT region can indeed be distinguished from normal WAT based on mitochondrial capacity. It is important to mention that mitochondrial respiration measurements in adipose tissue are not trivial, and only a few reports describe respiration measurements in human WAT. ${ }^{10,11}$ One of these studies shows that visceral adipose tissue is characterized by a $10-20 \%$ higher mitochondrial respiration when compared to subcutaneous adipose tissue..$^{10}$ In this study we measured cellular oxygen consumption in small adipose tissue samples and determined basal mitochondrial respiration and compared it to subcutaneous WAT taken from the same region. 


\section{Methods}

Approval was obtained from the medical ethical committee of the Maastricht University Medical Centre. Thirteen patients indicated for thyroid gland surgery (indications provided in Table 1) gave informed consent for perioperative AT from the BAT region and WAT biopsies. All subjects were euthyroid to rule out influence of thyroid hormone (Table 2).

\section{Biopsy procedure}

Previously successful UCP-1-positive adipose tissue biopsies were taken in the operative area involved in this type of surgery, ${ }^{2}$ and were here repeated. During surgery, both the surgeon (N.B.) and the researcher (G.V.) visually inspected the exposed operative area for presence of small 'islands' $( \pm 1-2 \mathrm{~cm})$ of AT from the BAT region along the sternocleidomastoid muscle and more cranially along the thyroid gland. These adipose tissue depots are typically light brown/beige and have a firm consistency compared to WAT. When AT from the BAT region was recognized a biopsy of $0.5 \mathrm{~cm}^{3}$ was taken together with $0.5 \mathrm{~cm}^{3}$ of WAT. AT biopsies showed both univacuolar and multivacuolar adipocytes, characterized as white adipocytes and brown/beige adipocytes, respectively (Figure 1).

\section{Tissue processing}

The tissue biopsies were directly placed in a tube with Phosphate-Buffer-Solution (PBS). Part of the AT from the BAT region and WAT ( $\pm 10 \%$ of the total biopsy) were snap-frozen in isopentane and stored at $-80^{\circ} \mathrm{C}$ until further analysis. The remaining AT from the BAT region and WAT was placed in separate $2 \mathrm{~mL}$ tubes with $10 \mathrm{mM} \mathrm{CaCl} 2$ solution and Collagenase solution (PBS, 1\% Bovine-SerumAlbumin and $0.1 \%$ Collagenase-type- 1 ) and then minced carefully. The tubes with minced AT from the BAT region and WAT were placed in a $37^{\circ} \mathrm{C}$ shaking water bath (75 RPM) for 30 minutes and then centrifuged for 5 minutes at 1200 RPM at room temperature. Then the tubes with tissue were loosely shaken manually to disrupt the pellet and centrifuged again. Subsequently, the separated top layer of both the AT from the BAT region and WAT was pipetted off in two separate tubes with $2.3 \mathrm{~mL}$ MiR05 respiration buffer (OROBOROS ${ }^{\circledR}$, Innsbruck, Austria). The interphase was removed and the pellet was also added to the respiration buffer. 
Table 1. Indications and surgical therapy per included subject. MENIIA-syndrome indicates the Multiple Endocrine Neoplasia syndrome type IIA, as was confirmed by genetic screening in subject families; a preventive, total thyroidectomy is indicated due to the high lifetime risk of developing thyroid gland cancer, mostly of the medullary type. Hyperparathyroidism indicates supraphysiological activity of the parathyroid gland, which is located adjacent to the thyroid gland itself. Parathyroidectomy indicates resection of the parathyroid gland, hemithyroidectomy indicates resection of the left/right half of the thyroid gland and total thyroidectomy indicates a total resection of the thyroid gland as a whole.

\begin{tabular}{lll} 
Subject & Indication & Surgery \\
\hline 1 & hyperparathyroidism & parathyroidectomy \\
2 & suspected thyroid gland mass & hemithyroidectomy \\
3 & suspected thyroid gland mass & hemithyroidectomy \\
4 & cyst left thyroid gland & hemithyroidectomy \\
5 & MENIIA-syndrome & total thyroidectomy \\
6 & suspected thyroid gland mass & hemithyroidectomy \\
7 & suspected thyroid gland mass & hemithyroidectomy \\
8 & MENIIA-syndrome & total thyroidectomy \\
9 & MENIIA-syndrome & total thyroidectomy \\
10 & struma & hemithyroidectomy \\
11 & hyperparathyroidism & parathyroidectomy \\
12 & struma & hemithyroidectomy \\
13 & suspected thyroid gland mass & hemithyroidectomy
\end{tabular}

Table 2. Subject characteristics for eight male and five female subjects indicated for thyroid gland surgery. BMI denotes Body Mass Index; TSH denotes Thyroid-Stimulating Hormone (normal range 0.4-3.5 mU/L), FT4 denotes plasma free thyroxine (normal range 4.0-18.0 $\mathrm{pmol} / \mathrm{L}$ ).

\begin{tabular}{ll} 
Parameter & Mean \pm S.D. \\
\hline Male / Female & $8 / 5$ \\
Age (years) & $43 \pm 19$ \\
Body mass $(\mathrm{kg})$ & $75.3 \pm 14.9$ \\
$\mathrm{BMI}\left(\mathrm{kg} / \mathrm{m}^{2}\right)$ & $25.7 \pm 4.9$ \\
$\mathrm{TSH}(\mathrm{mU} / \mathrm{L})$ & $2.1 \pm 1.4$
\end{tabular}

The respiration buffer containing the tissue was poured in two separate respiration chambers. Since the chamber volume is $\pm 2 \mathrm{~mL}$, the remaining $0.3 \mathrm{~mL}$ could be stored for determination of DNA and protein contents.

\section{Respiration measurements}

To determine oxygen consumption of AT from the BAT region and WAT, high-resolution respirometry was performed at $37^{\circ} \mathrm{C}$ by polarographic oxygen sensors in a two-chamber Oxygraph (OROBOROS $^{\circledR}$ Instruments, Innsbruck, Austria). Basal respiration was measured once the tissue preparation was added 
to the respiration chamber without addition of exogenous substrates for about 5-7 minutes. Once stable, mitochondrial respiration uncoupled from ATP synthesis (state 4) was determined via addition of the ATP synthase inhibitor oligomycin (1.0 $\mu \mathrm{L}, 2 \mathrm{mg} / \mathrm{mL})$ for about 4-5 minutes. In addition, saturating concentrations $(0.5 \mu \mathrm{L}$, $10 \mathrm{mM}$ ) of the chemical uncoupler FCCP (carbonyl-cyanide 4-(trifluoromethoxy)phenylhydrazone) were used to maximize oxygen flux, which allows for assessment of maximal mitochondrial capacity (state $u$ respiration). Baseline respiration, state 4 and state $u$ were measured according to Hoeks et al. ${ }^{12}$. After completion of respiration measurements, chamber-solutions were pipetted off and snap-frozen in liquid nitrogen and stored at $-80^{\circ} \mathrm{C}$ until further analysis. To account for any differences in the amount of collagenase-liberated cells added to the respiration chambers, the results were normalized to the DNA content measured spectrofluorometrically in the tissue preparations collected after measurement. ${ }^{13} \mathrm{All}$ raw oxygen fluxes (expressed as picomoles $\mathrm{O}_{2}$ consumption $\times$ seconds ${ }^{-1}$ ) for both WAT and AT from the BAT region were normalized for the DNA content measured in the cell suspensions derived from the oxygraph chambers $(\mathrm{mg} / \mathrm{mL})$. The DNA contents of these cell suspensions were not different for WAT and AT from BAT region $(0.037 \pm 0.014 \mathrm{mg} / \mathrm{mL}$ vs. $0.043 \pm 0.021 \mathrm{mg} / \mathrm{mL}, \mathrm{P}=0.108)$. Corrected oxygen fluxes are expressed as picomoles $\mathrm{O}_{2}$-consumption $\times$ seconds ${ }^{-1} \times(\mathrm{mg} / \mathrm{mL})^{-1}$. All results showed similar trends and significance when oxygen fluxes were uncorrected, or when corrected for cellular protein content (results not shown). For comparison of the relative decrease in oxygen consumption upon the addition of oligomycin, the oligomycin-insensitive leak-state-4 respiration was divided by baseline respiration. This uncoupling ratio (UCR) was not different in AT from the BAT region compared to WAT (AT from the BAT region; 0.83 \pm 0.17 , WAT; $0.79 \pm 0.20, P=0.501$ ). The relative contribution of baseline respiration and state 4 respiration to the maximally uncoupled respiration, as calculated by baseline respiration or state 4 respiration divided by state $u$, was higher in AT from the BAT region but this did not reach 


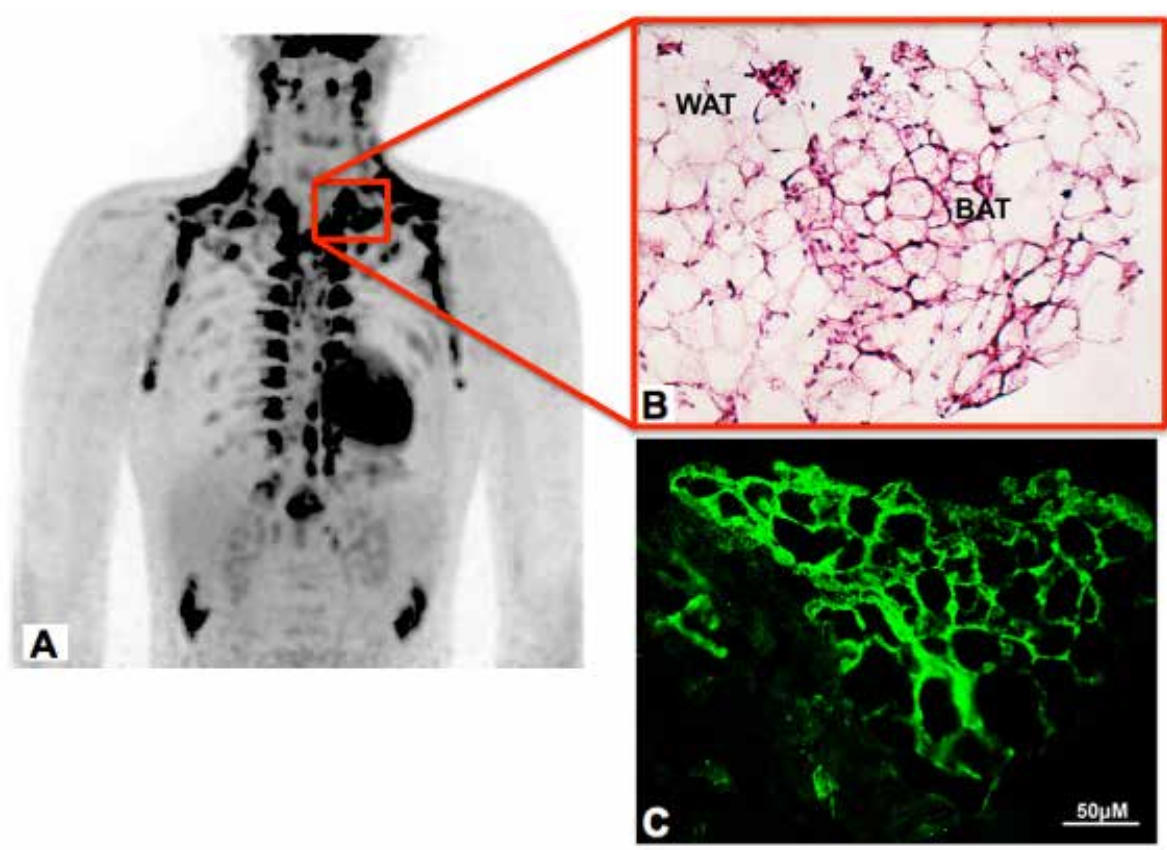

Figure 1. Histology of adipose tissue taken from the neck region during thyroid gland surgery. A: 18F-FDGPET-CT image of a lean young male subject after mild cold exposure. Black coloured areas indicate 18F-FDG glucose uptake. The red box indicates the anatomical adipose tissue area bordered by the medial edge of the sternocleidomastoid muscle, the lower edge of the thyroid gland and the jugulum. B: Hematoxylin-eosin (HE) section $(5 \mu \mathrm{m})$ of 'beige' coloured adipose tissue showing both univacuolar and multivacuolar adipocytes, characterized as white adipose tissue (WAT) and brown adipose tissue (BAT), respectively. C: Immunofluorescence-staining for uncoupling-protein-1 (UCP-1) was positive in the multivacuolar adipocytes, confirming the presence of brown adipocytes. BAT denotes brown adipose tissue and WAT denotes white adipose tissue. Shown histology derives from a perioperative biopsy taken during a preventive total thyroidectomy for MENIIA-syndrome in a 19 -year old male subject with a BMI of $21.9 \mathrm{~kg} / \mathrm{m} 2$.

statistical significance (baseline / state u; AT from the BAT region; 0.83 \pm 0.21 , WAT; $0.70 \pm 0.33, P=0.292$, state 4 / state $u$; AT from the BAT region; $0.68 \pm 0.17$, WAT; $0.54 \pm 0.26, P=0.122$ ).

\section{Statistical analysis}

Reported data was expressed as means \pm SD. Statistical analyses were performed with PASW Statistics 18.0 for Mac OS X 10.6.4. WAT and BAT respiration measurements within subjects were compared using paired student's t-tests. Comparisons between for age groups were made using unpaired student's t-tests. A P-value below 0.05 was considered significant. 


\section{Results}

\section{Presence of UCP-1 in adipose tissue biopsies}

In the adipose tissue biopsies from the BAT region additional immunofluorescence staining for the brown/beige adipose tissue-specific uncoupling protein 1 (UCP-1, Figure 1) was positive in the multivacuolar adipocytes, confirming the presence of brown/beige adipocytes in this adipose tissue depot. UCP-1 immunofluorescence for the WAT biopsies was completely negative, confirming no UCP-1 was present in the subcutaneous WAT biopsies.

\section{Adipose tissue respirometry in AT from the BAT region compared to WAT}

Figure 2 shows the respiration levels of subcutaneous (WAT) and AT from the BAT region after normalization for DNA. Baseline respiration without addition of substrates was more than three times higher in AT from the BAT region compared to WAT (430.9 \pm 410.3 vs. 132.6 $\pm 90.8 \quad \mathrm{O}_{2}$ flux, $\left.\mathrm{P}=0.017\right)$. After addition of oligomycin to achieve leak state 4 respiration, oxygen fluxes for both AT from the BAT region and WAT significantly decreased (baseline vs. state 4; AT from the BAT region; $430.9 \pm 410.3$ vs. $364.1 \pm 367.7 \mathrm{O}_{2}$ flux, $\mathrm{P}=0.002$, for WAT; $132.6 \pm 90.8$ vs. $104.6 \pm 78.6 \mathrm{O}_{2}$ flux, $\left.\mathrm{P}<0.001\right)$. Upon titration of FCCP oxygen fluxes increased to maximally uncoupled respiration (increase after FCCP; for AT from the BAT region; $364.1 \pm 367.7$ to $491.1 \pm 423.8 \mathrm{O}_{2}$ flux, $\mathrm{P}<0.001$, for WAT; $104.6 \pm 78.6$ to 189.4 $\pm 123.2 \mathrm{O}_{2}$ flux, $\left.\mathrm{P}=0.003\right)$.

\section{Age differences in respiration control ratios}

The relative contribution of state 4 to state $u$ respiration in WAT was significantly correlated to age $\left(r^{2}=0.466, P=0.010\right.$, Figure 3$)$. For AT from the BAT region, the ratio of state 4 vs. state $u$ for AT from the BAT region was significantly higher for subjects aged under 50 years $(n=6)$ compared to older subjects $(\geq 50$ years, $n=7$, state 4 /state $u ; 0.78 \pm 0.11$ vs. $0.60 \pm 0.17 \mathrm{O}_{2}$ flux, $P=0.042$, Figure 3 ).

\section{Discussion}

In this study oxygen consumption of adipose tissue from the human BAT region was compared to subcutaneous white adipose tissue, taken from the same anatomical region in the neck. The respiration in AT from the BAT region was on average 


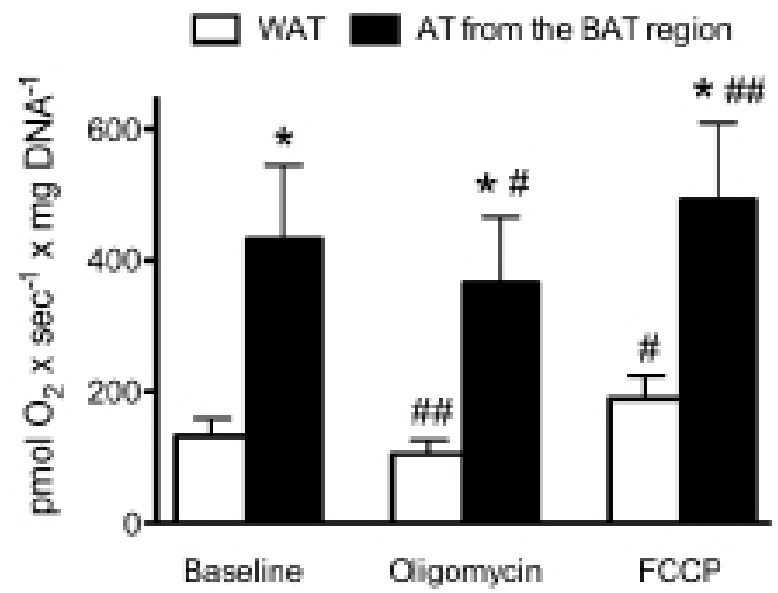

Figure 2. Cellular respiration levels of human white adipose tissue (WAT) and adipose tissue (AT) taken from regions shown to contain brown adipose tissue (BAT). Oxygen consumption in WAT and AT from the BAT region shown for baseline respiration, oligomycin-insensitive leak state 4 respiration upon addition of oligomycin and maximally uncoupled state u respiration after addition of FCCP. All uncorrected oxygen fluxes (expressed as picomoles $\mathrm{O} 2$ consumption $\mathrm{x}$ seconds-1) for both AT from the BAT region and WAT were normalized for the DNA content in chamber solutions $(\mathrm{mg} / \mathrm{mL})$. Values shown are means + S.E.M. ${ }^{\star} \mathrm{P}<0.05$ for comparison between AT from the BAT region versus WAT baseline, oligomycin and FCCP-stimulated oxygen fluxes, respectively. $\# \mathrm{P}<0.05$, \#\# $<0.001$ for comparison between baseline vs. oligomycin and oligomycin vs. FCCP-stimulated oxygen fluxes within tissues for WAT and AT from the BAT region.

three times higher compared to subcutaneous WAT. These findings are in line with previous animal studies that show mitochondrial respiration in BAT is relatively high. ${ }^{14}$

Recently detailed metabolic imaging studies on BAT activity in man using PET-CT have been performed. ${ }^{3,6,7}$ Upon cold exposure BAT oxidative metabolism as determined using ${ }^{11} \mathrm{C}$-acetate uptake increased $\pm 150 \%$ whereas BAT perfusion using $\left(\left({ }^{15} \mathrm{O}\right) \mathrm{H}_{2} \mathrm{O}\right)$ increased $\pm 200 \%$ and glucose uptake using ${ }^{18} \mathrm{~F}$-FDG increased $\pm 600 \% .{ }^{6,7}$ The same parameters did not change significantly in WAT and baseline BAT oxygen uptake and perfusion were already doubled compared to WAT. ${ }^{6,7}$ Here we show that tissue taken from the region identified to be BAT already uses $300 \%$ more oxygen in the basal state and thus displays increased cellular respiration. This $300 \%$ higher basal respiration is much higher than the previous reported $10-20 \%$ higher respiration in visceral adipose tissue compared to WAT, indicating that in humans AT from the BAT region is a very special metabolic active adipose tissue depot. ${ }^{10}$ It is important to note that biopsies from the neck region do not solely consist of brown or beige adipocytes, as may be the case in more homogenous BAT depots in rodents. Rather, as observed in Figure 1 and before, ${ }^{15}$ the adipose tissue 
of this region is a mixture of white and UCP1-positive adipocytes, with a varying composition between subjects. This indicates that mitochondrial respiration in pure brown/beige adipocytes is likely much higher, and illustrates that strategies that are able to induce human brown/beige adipocytes may effectively result in an adipose tissue depot with a very high metabolic potential. Due to the limited amount of human tissue available and the amount needed for respiration studies, we could not perform a further characterization of the neck adipose tissue, such as mitochondrial content, which most likely explains the higher basal metabolic activity. More important, it is generally thought that the main component of heat production (extra oxygen consumption) in BAT derives from UCP1-mediated uncoupled 'leak' respiration. ${ }^{16}$ The UCP-1 mediated thermogenesis needs to be activated by external stimuli, e.g. a sympathetically induced norepinephrine-release. Indeed,

A
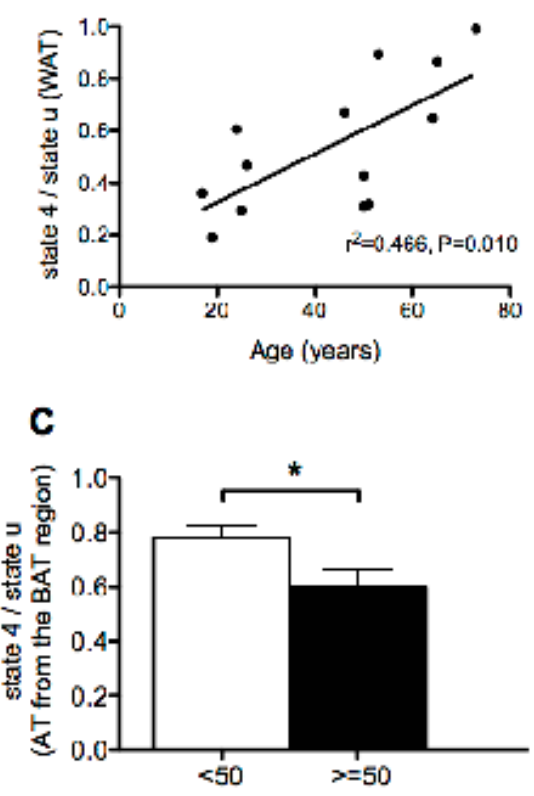

B

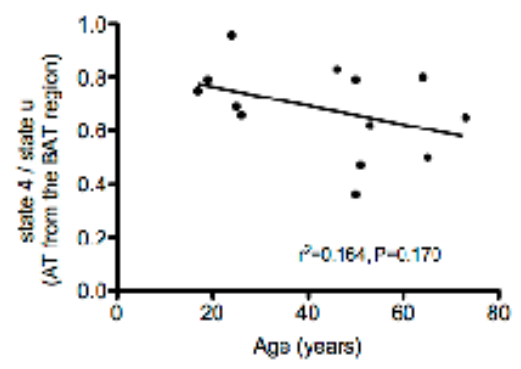

Figure 3. Relationships between subject age and mitochondrial respiration states of WAT and AT from the BAT region. A: Relationship between age and the relative contribution of state 4 leak respiration to maximally uncoupled state u respiration in WAT. B: Relationship between age and the relative contribution of state 4 leak respiration to maximally uncoupled state $\mathrm{u}$ respiration in AT from the BAT region. C: Comparison of the relative contribution of state 4 leak respiration to maximally uncoupled respiration in AT from the BAT region from subjects below and above 50 years of age. ${ }^{\star} \mathrm{P}<0.05$ 
in mice maximal respiration in BAT upon addition of NE increases 7-8 fold over basal respiration, ${ }^{17}$ and thus much higher than the FCCP-induced respiration in our study. Unfortunately, we were unable to determine NE-stimulated mitochondrial respiration in the isolated adipocytes from the BAT region: initial attempts were not successful, and the limited amount of this valuable human tissue available did not allow optimization of the procedure. In that respect, it is also known from rodent studies that BAT from several (e.g. 6-8 mice) animals needs to be pooled to render isolated brown adipocytes that respond to NE ex vivo. Nevertheless, the reported basal respiration levels in AT from the BAT region, together with the fact that this tissue is inhomogeneous with respect to white and brown/beige fat cell composition, suggest that true respiration in pure human brown/beige adipocytes may be high.

Interestingly, the contribution of state 4 to state $u$ in AT from the BAT region was higher in subjects $<50$ years, which would be consistent with the notion that younger subjects are suggested to possess more active BAT. ${ }^{18}$

Recent reports show that within WAT there is a subpopulation of cells that has the capacity to become thermogenic. ${ }^{19,20}$ These 'beige' or BRITE (brown-in-white) cells possess UCP-1 and are thus capable of thermogenesis upon sufficient stimulation. ${ }^{20}$ Several studies have now reported the development of beige/BRITE cells upon diverse stimuli. ${ }^{20-23}$ In vitro cultured human preadipocytes that were isolated from adipose tissue taken in the same anatomical region as in this study became thermogenic adipocytes upon stimulation by both $ß$-agonists and proliferatoractivated-receptor-gamma (PPAR-gamma). Intriguingly, we recently showed that adipose tissue taken from the same area as in this report in fact is composed of such 'beige cells', genetically distinct from white and brown fat. ${ }^{9}$ Respiration analysis of cloned beige cells showed that respiratory activity of these cells is comparable to classical brown adipocytes. ${ }^{9}$ These findings suggest that the amount of "brown adipose tissue" in humans may be inducible when activated by the right stimulators, consistent with our recent finding of induction of human "BAT" after weight loss. ${ }^{24}$ In summary, we here show that the basal mitochondrial respiration of adipose tissue from the region characterized as "BAT" can be distinguished from subcutaneous adipose tissue by a $\sim 300 \%$ higher mitochondrial respiration. This illustrates that AT from human BAT region is a distinct adipose tissue depot with high mitochondrial capacity. Future studies should be directed to further study the metabolic and bioenergetic aspects of this interesting depot. Cultured adipocytes from this AT 
depot may be of specific interest to identify molecular targets that are able to stimulate this tissue. Such studies could play an important role in obesity treatment by inducing BAT activity and increasing energy expenditure. 


\section{References}

1. Nedergaard J, Bengtsson T, Cannon B. Unexpected evidence for active brown adipose tissue in adult humans. Am J Physiol Endocrinol Metab 2007;293:E444-52.

2. van Marken Lichtenbelt WD, Vanhommerig JW, Smulders NM, et al. Cold-activated brown adipose tissue in healthy men. N Engl J Med 2009;360:15008.

3. Virtanen KA, Lidell ME, Orava J, et al. Functional brown adipose tissue in healthy adults. N Engl J Med 2009;360:1518-25.

4. Yoneshiro T, Aita S, Matsushita M, et al. Brown Adipose Tissue, WholeBody Energy Expenditure, and Thermogenesis in Healthy Adult Men. Obesity (Silver Spring) 2011;19:13-6.

5. Vijgen GH, Bouvy ND, Teule GJ, Brans B, Schrauwen P, van Marken Lichtenbelt WD. Brown adipose tissue in morbidly obese subjects. Plos One 2011;6:e17247.

6. Orava J, Nuutila P, Lidell ME, et al. Different metabolic responses of human brown adipose tissue to activation by cold and insulin. Cell Metab 2011;14:272-9.

7. Ouellet $\mathrm{V}$, Labbe $S M$, Blondin DP, et al. Brown adipose tissue oxidative metabolism contributes to energy expenditure during acute cold exposure in humans. J Clin Invest 2012;122:545-52.

8. Lee P, Swarbrick MM, Ting Zhao J, Ho KK. Inducible Brown Adipogenesis of Supraclavicular Fat in Adult Humans. Endocrinology 2011.

9. Wu J, Bostrom P, Sparks LM, et al. Beige adipocytes are a distinct type of thermogenic fat cell in mouse and human. Cell 2012;150:366-76.

10. Kraunsoe $\mathrm{R}$, Boushel $\mathrm{R}$, Hansen $\mathrm{CN}$, et al. Mitochondrial respiration in subcutaneous and visceral adipose tissue from patients with morbid obesity. J Physiol 2010;588:2023-32.

11. Hallgren $\mathrm{P}$, Korsback $\mathrm{S}$, Sjostrom L. Measurements of adipose tissue respiration in a closed chamber using an oxygen sensor: methodological considerations. J Lipid Res 1986;27:996-1005.

12. Hoeks J, van Herpen NA, Mensink $M$, et al. Prolonged fasting identifies skeletal muscle mitochondrial dysfunction as consequence rather than cause of human insulin resistance. Diabetes 2010;59:2117-25.

13. Labarca C, Paigen K. A simple, rapid, and sensitive DNA assay procedure. Annal Biochem 1980;102:344-52.

14. Fain JN, Reed N, Saperstein R. The isolation and metabolism of brown fat 
cells. J Biol Chem 1967;242:1887-94.

15. Zingaretti MC, Crosta F, Vitali A, et al. The presence of UCP1 demonstrates that metabolically active adipose tissue in the neck of adult humans truly represents brown adipose tissue. FASEB J 2009;23:3113-20.

16. Cannon B, Nedergaard J. Brown adipose tissue: function and physiological significance. Physiol Rev 2004;84:277-359.

17. Matthias A, Ohlson KB, Fredriksson JM, Jacobsson A, Nedergaard J, Cannon B. Thermogenic responses in brown fat cells are fully UCP1-dependent. UCP2 or UCP3 do not substitute for UCP1 in adrenergically or fatty scid-induced thermogenesis. J Biol Chem 2000;275:25073-81.

18. Saito $M$, Okamatsu-Ogura $Y$, Matsushita $M$, et al. High Incidence of Metabolically Active Brown Adipose Tissue in Healthy Adult Humans: Effects of Cold Exposure and Adiposity. Diabetes 2009;58:1526-31.

19. Kajimura S, Seale P, Spiegelman BM. Transcriptional control of brown fat development. Cell Metab 2010;11:257-62.

20. Petrovic N, Walden TB, Shabalina IG, Timmons JA, Cannon B, Nedergaard J. Chronic peroxisome proliferator-activated receptor gamma (PPARgamma) activation of epididymally derived white adipocyte cultures reveals a population of thermogenically competent, UCP1-containing adipocytes molecularly distinct from classic brown adipocytes. J Biol Chem 2009;285:7153-64.

21. Seale P, Conroe HM, Estall J, et al. Prdm16 determines the thermogenic program of subcutaneous white adipose tissue in mice. J Clin Invest 2011;121:96105.

22. Schulz TJ, Huang TL, Tran TT, et al. Identification of inducible brown adipocyte progenitors residing in skeletal muscle and white fat. Proc Natl Acad Sci U S A 2011;108:143-8.

23. Bostrom $\mathrm{P}, \mathrm{Wu}$ J, Jedrychowski MP, et al. A PGC1-alpha-dependent myokine that drives brown-fat-like development of white fat and thermogenesis. Nature 2012;481:463-8.

24. Vijgen GH, Bouvy ND, Teule GJ, et al. Increase in Brown Adipose Tissue Activity after Weight Loss in Morbidly Obese Subjects. J Clin Endocrinol Metab 2012. 


$$
\theta_{-1}
$$




\section{Chapter 7}

\section{Vagus nerve stimulated increased energy expenditure in relation to brown adipose tissue activity}




\section{Abstract}

\section{Background}

Human brown adipose tissue (BAT) activity is inversely related to obesity and positively related to energy expenditure. BAT is highly innervated and it is suggested the vagus nerve mediates peripheral signals to the central nervous system, there connecting to sympathetic nerves that innervate BAT. Vagus nerve stimulation (VNS) is used for refractory epilepsy, but is also reported to generate weight loss. We hypothesize VNS increases energy expenditure by stimulating BAT activity.

\section{Methods}

Fifteen patients with stable VNS therapy (age: $45 \pm 10 y$ rs; body mass index; $25.2 \pm 3.5$ $\mathrm{kg} / \mathrm{m}^{2}$ ) were included. Ten subjects were measured twice, once with active and once with inactivated VNS. Five other subjects were measured twice, once with active VNS at room temperature and once with active VNS under cold exposure in order to determine maximal cold-induced BAT activity.

\section{Results}

Basal metabolic rate (BMR) was significantly higher when VNS was turned on (mean change; $+2.2 \%$ ). Mean BAT activity was not significantly different between active VNS and inactive VNS (BAT SUVMean; $0.55 \pm 0.25$ versus $0.67 \pm 0.46, P=0.619$ ). However, the change in energy expenditure upon VNS intervention (On-Off) was significantly correlated to the change in BAT activity $(r=0.935, \mathrm{P}<0.001)$.

\section{Conclusions}

Activated VNS significantly increased energy expenditure. The observed change in energy expenditure was significantly related to the change in BAT activity. This suggests that the VNS-induced increase energy expenditure in humans is at least partly due to BAT activation. Chronic VNS might have a beneficial effect on the energy balance and play an important role in weight management. 


\section{Introduction}

Obesity results from an imbalance between energy intake and energy expenditure and most applied obesity therapies are focussed on decreasing energy intake. ${ }^{1}$ However, in addition to a reduction in energy intake, increasing energy expenditure could be an effective means to prevent or treat obesity. In rodents, activated brown adipose tissue (BAT) is the main contributor to the regulated energy expenditure. ${ }^{2}$ Here, during cold exposure BAT activation increases energy expenditure by producing heat (thermogenesis) to prevent hypothermia. ${ }^{2}$ Recently functional brown adipose tissue (BAT) was also shown in adult man by means of FDG-PETCT $\quad\left({ }^{18} \mathrm{~F}\right.$-fluorodeoxyglucose positron-emission tomography and computed tomography). ${ }^{3,4}$ Interestingly, BAT activity was inversely correlated with body mass index (BMI) and body fat percentage (BF\%). 3,5 Moreover, subjects with active BAT show a significantly higher energy expenditure compared to subjects without BAT activity. ${ }^{5,6}$ This suggests BAT is related to the development of obesity and increasing BAT activity could be a new treatment modality for obesity. Rodent studies and histological analyses of human tissue samples have shown BAT is highly sympathetically innervated. 7,8 Stimulation of sympathetic b-receptors on brown adipocytes was already suggested as a possible target for the treatment of obesity several years ago' ${ }^{9}$. More recent studies report stimulation and suppression of BAT activity in rodents which is mediated through the vagus nerve. ${ }^{10,11}$ It is suggested the vagus nerve mediates peripheral signals to the central nervous system (CNS), which in turn connects to the sympathetic nerves that innervate BAT.11 Interestingly, electrical stimulation of the cervical part of the vagus nerve (vagus nerve stimulation (VNS)) generates weight loss in rodents. ${ }^{12,13}$ Weight loss was also reported as a secondary outcome of VNS in humans, where it is used to treat refractory epilepsy. ${ }^{14-19}$ The weight loss induced by VNS may be the result of increased energy expenditure through BAT stimulation. However, most human trials are primarily focused on the effect of VNS on epilepsy from a neurological viewpoint and to date no prospective study has investigated the effect of VNS on energy expenditure. Therefore, this study aims to define the relation between VNS energy expenditure and BAT activation in a patient cohort on chronic stable VNS therapy for refractory epilepsy. We hypothesize that VNS increases energy expenditure by stimulating BAT activity. 


\section{Methods}

Approval for the study protocol was obtained from the ethics committee of the Maastricht University Medical Centre. The study was registered in the Clinical Trial Register under the ClinicalTrials.gov Identifier NCT01491282. Written informed consent was obtained from 15 patients on stable VNS therapy using the Vagus Nerve Stimulation Therapy System (VNS Therapy ${ }^{\circledR}$, Cyberonics Inc., Houston, Texas, USA) for refractory epilepsy. All included subjects had stable therapy (i.e. no recent adjustments in VNS settings or epilepsy medication (Table 1) and were aged between 18 and 65 years. Exclusion criteria were daily epileptic seizures, pregnancy, ketogenic diet, mental retardation and psychological instability.

\section{Principle of vagus nerve stimulation}

VNS is an approved procedure for refractory epilepsy patients. The therapeutic goal of VNS is a reduction in seizure frequency, seizure severity, and an improvement in general well-being. The VNS Therapy ${ }^{\circledR}$ System consists of a programmable pulse generator implanted subcutaneously at the level of the upper left chest. A helical bipolar electrode connected to the left vagus nerve transmits electric signals generated by the pulse generator. The stimulation parameters are adjusted via telemetric control to an optimal value for every individual patient. Standard starting parameters are: output current $0.25 \mathrm{~mA}$, pulse width $250-500 \mathrm{msec}$, frequency $30 \mathrm{~Hz}$, On-period $30 \mathrm{sec}$, Off-period $5 \mathrm{~min}$. If the effect is suboptimal, the output current is increased in steps of $0.25 \mathrm{~mA}$ to a maximum of $3 \mathrm{~mA}$. The most common standard therapeutic setting is: output current 1-2 mA, pulse width $250 \mathrm{msec}$, frequency $30 \mathrm{~Hz}$, On-period $30 \mathrm{sec}$, Off-period $5 \mathrm{~min}$. A second option is the introduction of Rapid Cycling, where the On-period is $7 \mathrm{sec}$ and the subsequent Off-period $20 \mathrm{sec}$. Patients responding to Rapid Cycling are thought to need this extra stimulation to acutely suppress an upcoming seizure in order to obtain a reduction in their seizure frequency.

\section{Study protocol}

Energy expenditure was measured using indirect calorimetry and BAT activity was assessed by means of FDG-PET-CT during actual VNS and when VNS was inactivated. This was possible since the anticonvulsive effect of VNS is considered chronic and short-term inactivation does not lead to an acute increase in seizures. In addition, we compared BAT activity during VNS and during mild cold stimulation. The mild cold 
intervention served as a control since it is known to activate BAT. ${ }^{3,5}$ Ideally, one would like to compare the effect of active/inactive VNS with and without cold exposure, which would result in four FDG-PET-CT measurements in each subject. However, due to ethical considerations a maximum of two FDG-PET-CT measurements was allowed. Therefore, ten patients were measured in thermoneutral (TN) conditions with active (VNS-On) and inactive VNS (VNS-Off) respectively. In addition, five subjects were measured with active VNS in TN conditions (VNS-TN) and during mild cold exposure (VNS-Cold) respectively (Table 2). Subjects were measured under fasted conditions (no food intake from 10 p.m. the night before, only water consumption was allowed) from 9 a.m. to 2 p.m. under supervision of a specialized research nurse (L.L.). VNS-On and VNS-Off took place on separate occasions Table 1. Medication use per patient.

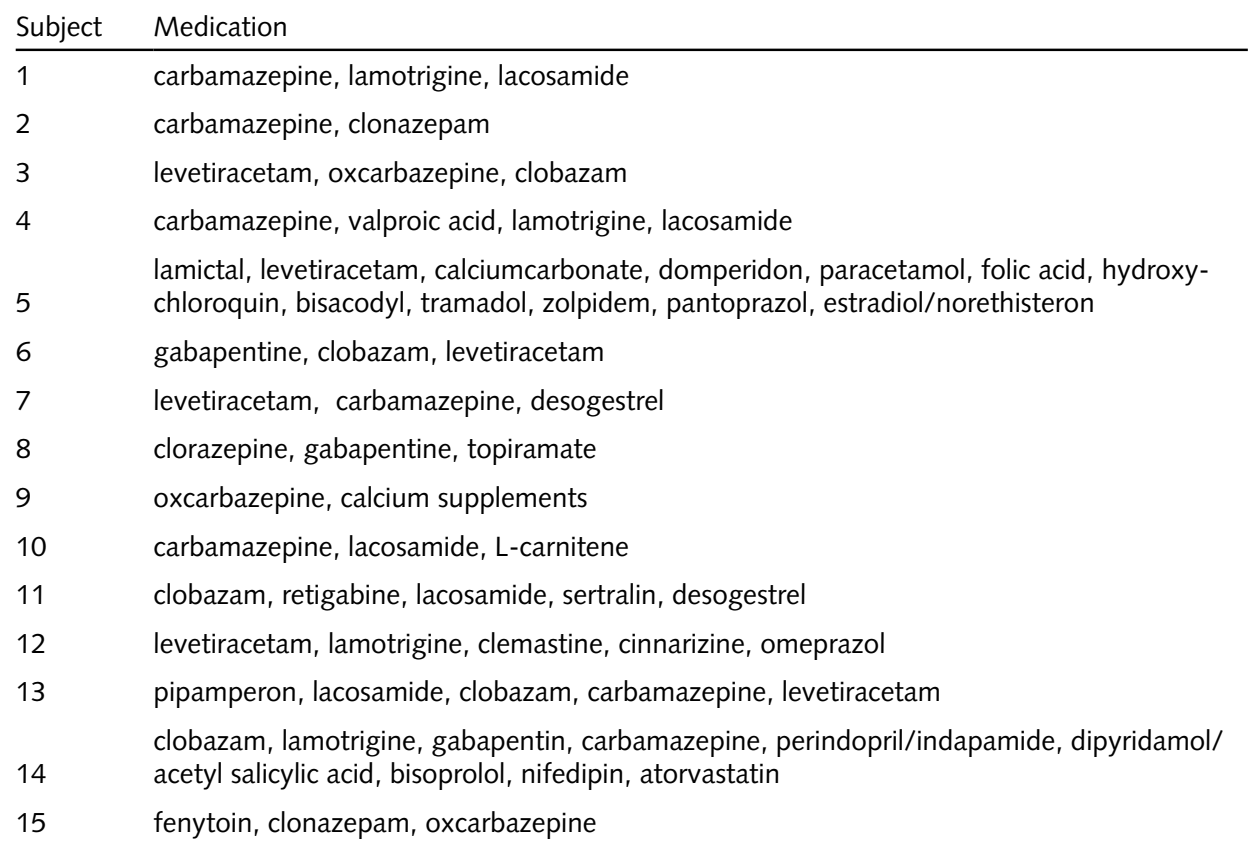

within 14 days. During VNS-Off the system was inactivated (output current $0 \mathrm{~mA}$, magnet function $0 \mathrm{~mA}$ ) at 9:30 a.m. prior to the measurements. At the end of the test day (2:00 p.m.) the VNS system was re-activated. During VNS-Cold the settings of the VNS system were not adjusted. VNS-TN and VNS-Cold were also 
performed within a 14-day period. Body composition (body fat\%, fat mass (FM), fat free mass (FFM)) was determined by dual x-ray absorptiometry (DXA, type Discovery A, Hologic, Bedford, MA, USA).

During the experiments the subjects wore standardized clothing (socks 0.02 clo (clothing insulation factor), shirt 0.09 clo, sweatpants 0.28 clo, underwear 0.04 clo, total clo factor $0.43 \mathrm{clo}){ }^{20} \mathrm{~A}$ catheter was placed in the left antecubital vein for blood sampling during measurements and to inject ${ }^{18} \mathrm{~F}$-Fluoro-Deoxy-Glucose (FDG) for FDG-PET-CT (for further detail on scanning protocol see below). Body core temperature was monitored by a telemetric pill that was orally ingested (CoreTemp, HQ Inc., Palmetto, FL, USA). iButtons were placed at 14 ISO-defined skin sites to measure skin temperatures. ${ }^{21}$ For comparison between proximal and distal body temperatures, the proximal skin temperature was defined as the mean of the iButtons placed on the chest, abdomen, shoulder and lower back. The distal skin temperature was defined as the mean of the iButtons placed on the wrist and foot. Next, subjects were placed in a semi-supine position on a nephrodialysis chair covered with a water-perfused mattress (necessary for the cold exposure protocol, see below) in a specially equipped tent (Colorado Altitude Training, Louisville, CO, USA) where energy expenditure was measured by indirect calorimetry using a ventilated hood system (Omnical, Maastricht Instruments BV, Maastricht, the Netherlands). Laser Doppler Flowmetry (PF5000; Perimed, Jarfalla, Sweden) was used to determine skin blood flow gradients dorsal of the medial malleolus and at the glabrous skin of the ventral hand thenar to monitor whether a subject was in its thermoneutral zone as reported. ${ }^{22}$ The VNS-On/Off $(n=10)$ and VNS-TN $(n=5)$ measurements were performed under thermoneutral conditions (average room temperature VNS-On; $24.9 \pm 0.8^{\circ} \mathrm{C}$, VNS-Off; $25.2 \pm 1.0^{\circ} \mathrm{C}$, VNS-TN; $25.2 \pm 0.9^{\circ} \mathrm{C}$, $\mathrm{P}=0.7260$ ). After the first hour of measuring under thermoneutral conditions (t0-t60) 10ml FDG was injected. After the second hour (t60-t120), FDG-PETCT imaging was performed to quantify metabolically active BAT. The scanning protocol, FDG activity (74 MBq) and data analysis were identical to our previous studies on BAT activity.,23 During VNS-Cold a personal cooling protocol was used as described earlier in detail. ${ }^{5}$ In short, after measuring under thermoneutral conditions ( $\mathrm{t0}-\mathrm{t} 30$ ), the subject was cooled until they reported shivering. Upon the first signs of shivering the temperature was raised slightly (on average $2.3 \pm 0.6^{\circ} \mathrm{C}$ ) to 
ensure only non-shivering thermogenesis (NST). ${ }^{5}$ After one hour of stable cooling situation (t30-t90), 10ml FDG was injected, followed by the second hour of cooling (t90-t150) and subsequent FDG-PET-CT imaging.

\section{FDG-PET-CT analysis}

Tissue activity on FDG-PET-CT was expressed in standard uptake value (SUV; as calculated by uptake (kilobequerels per $\mathrm{ml}$ ) per injected dose (kilobequerels) per patient weight (grams)) using the PMOD Biomedical Image Quantification tool (PMOD Technologies Ltd., Zurich, Switzerland). Tissue activity of each region was determined by the mean SUV uptake (SUVMean). For comparison, standard cube volumes-of-interest (VOIs) were placed in the tissue type studied: Brown Adipose Tissue (BAT), deltoid muscle (Deltoid), biceps muscle (Biceps), triceps muscle (Triceps), erector spinae muscle at the level of vertebrae C7 (C7), T8 (T8), and L3 (L3), the mean of all analyzed muscles (Muscle), subcutaneous white adipose tissue (WAT Sc), visceral white adipose tissue (WAT Visc), the liver (Liver) and the brain at the level of the cerebellum (Brain) (Figure 1). The volume of these threedimensional cubes was $1.33 \mathrm{~cm}^{3}$ (i.e. three consecutive slices of $0.44 \mathrm{~cm}^{3}$ thickness). For BAT the placement of one cube in supraclavicular adipose tissue often interferes with adjacent muscle and hence the cube volume in BAT was composed of three separate slices of $1.0 \times 1.0 \times 0.44 \mathrm{~cm}$. When drawing the cubes, attention was paid to the Hounsfield Units ( $\mathrm{HU}$ ) on CT to define the specific tissue type and to avoid interference with adjacent tissue. For example, adipose tissue has a $\mathrm{HU}$ of -200 to -10 and muscle 10 to 100. Finally, FDG-PET-CT imaging during the Off-measurement failed in one subject due to a critical image reconstruction error. Consequently, values shown are for nine subjects in the VNS-On/Off group and five subjects in the VNS-TN/Cold group.

\section{Statistics}

Reported data were expressed as means \pm S.D. Statistical analyses were performed with PASW Statistics 18.0 for Mac OS X 10.6.4. Repeated measurements (VNS-On versus VNS-Off, and VNS-TN versus VNS-Cold) were compared using paired student's t tests. Comparisons between groups (VNS-On/Off versus VNS-TN/ Cold) were made using one-way ANOVA with a post-hoc Bonferroni correction. A $\mathrm{P}$-value below 0.05 was considered significant. 


\section{Results}

\section{Subject characteristics}

Ten male and five female patients with a mean age of $45 \pm 10$ years and a mean Body Mass Index (BMI) of $25.2 \pm 3.5 \mathrm{~kg} / \mathrm{m}^{2}$ that were (successfully) treated with VNS for refractory epilepsy were included (Table 1). VNS implantation was on average 59 \pm 19 months (range; $22-89$ months) ago and all subjects did not have any recent adjustments in their VNS settings or medication (last adjustment $>1$ month ago, VNS output current; $1.58 \pm 0.55 \mathrm{~mA}$, Table 2). Pre-VNS treatment body weight and $B M I$ were retraceable for 11 subjects and were not significantly different from weight and $\mathrm{BMI}$ during the study (implant weight and $\mathrm{BMl} ; 71.2 \pm 12.5 \mathrm{~kg}, 24.7 \pm 3.4$ $\mathrm{kg} / \mathrm{m}^{2}$, current weight and $\left.\mathrm{BMl} ; 72.9 \pm 11.6 \mathrm{~kg}, 25.2 \pm 3.5 \mathrm{~kg} / \mathrm{m}^{2}, \mathrm{P}=0.414\right)$. The subject characteristics were not different for the On/Off $(n=10)$ versus the TN/ Cold group $(n=5)$ (Table 2 ).

\section{Energy expenditure}

Basal metabolic rate (BMR) decreased significantly when VNS was turned off $(68.6 \pm 7.9 \mathrm{~J} / \mathrm{s}$ versus $67.2 \pm 8.1 \mathrm{~J} / \mathrm{s}, \mathrm{P}=0.038$, mean change; $2.2 \%$, range; -3.1 to $7.8 \%$, Table 3, Figure 1). All but one subject decreased their BMR; the male subject with increased BMR was very restless during the Off-measurement due to personal affairs. Exclusion of this subject revealed energy expenditures of $68.6 \pm 7.9 \mathrm{~J} / \mathrm{s}$ (VNS-On) and $66.6 \pm 8.31 \mathrm{~J} / \mathrm{s}$ (VNS-Off) $(P=0.005)$. The subject's data remained included in the further presented analyses.

Upon cold exposure BMR increased as compared to thermoneutral conditions (TN; $68.2 \pm 1.5 \mathrm{~J} / \mathrm{s}$ versus Cold; $75.1 \pm 7.7 \mathrm{~J} / \mathrm{s}, \mathrm{P}=0.107$, Table 3 ). BMR in the VNS-TN group was similar to $B M R$ in the VNS-On group $(P=0.919)$.

\section{Core and skin temperatures}

Body core and mean skin temperatures did not change significantly when VNS was turned off (core temperature; $37.0 \pm 0.3{ }^{\circ} \mathrm{C}$ versus $36.9 \pm 0.4{ }^{\circ} \mathrm{C}$, mean skin temperature; $33.5 \pm 0.8{ }^{\circ} \mathrm{C}$ versus $33.6 \pm 0.7{ }^{\circ} \mathrm{C}, \mathrm{P}=0.738$, Table 2). When 
skin temperatures were divided in proximal and distal zones ${ }^{21}$, the distal skin temperature was significantly increased during the Off-measurement $\left(31.7 \pm 1.6^{\circ} \mathrm{C}\right.$ versus $33.0 \pm 1.7^{\circ} \mathrm{C}, \mathrm{P}=0.016$, Table 2 ).

During cold exposure skin temperatures decreased significantly (TN: mean skin temperature; $33.2 \pm 0.4{ }^{\circ} \mathrm{C}$ versus Cold: $29.0 \pm 0.8^{\circ} \mathrm{C}, \mathrm{P}<0.001$, Table 2).

\section{Skin perfusion}

Perfusion of the medial foot and ventral hand did not change during VNS (foot; 8.2 \pm 3.9 Arbitrary Units (AU) versus 13.4 $\pm 21.4 \mathrm{AU}, \mathrm{P}=0.396$, hand; $54.5 \pm 35.3$ $\mathrm{AU}$ versus $81.7 \pm 70.5 \mathrm{AU}, \mathrm{P}=0.149$, Table 3 ). Cold exposure significantly decreased

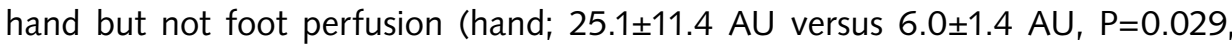
foot; 9.6 $\pm 8.0 \mathrm{AU}$ versus $6.8 \pm 4.4 \mathrm{AU}, \mathrm{P}=0.396$, Table 3 ).

\section{BAT activity and FDG uptake in other tissues}

Figure 2 shows representative images of FDG-uptake on PET-CT in the studied groups. The mean SUV for BAT showed no statistical difference during VNS (BAT SUVMean; $0.55 \pm 0.25$ versus $0.67 \pm 0.46, P=0.619$, Table 2 , Figure 3 ). In different muscles analyzed, the triceps muscle had a significantly increased FDG-uptake when VNS was turned off (Triceps SUVMean; $0.51 \pm 0.14$ versus $0.70 \pm 0.16, P=0.024$, Table

Table 2. Subject characteristics for all subjects, the intervention group with Vagus Nerve Stimulator (VNS) On and Off $(n=10)$ and for the group with VNS during thermoneutral (VNS-TN) conditions and cold exposure (VNS-Cold) $(\mathrm{n}=5)$. P-values shown for unpaired $\mathrm{t}$-tests between VNS-On/Off and VNS-TN/Cold. ${ }^{*} \mathrm{P}<0.05$

\begin{tabular}{lcccc} 
Characteristics & Group & VNS-On/Off & VNS-TN/Cold & P-value \\
\hline $\mathrm{n}$ & 15 & 10 & 5 & \\
Sex (Male/Female) & $10 \mathrm{M} / 5 \mathrm{~F}$ & $4 \mathrm{M} / 6 \mathrm{~F}$ & $1 \mathrm{M} / 4 \mathrm{~F}$ & \\
Age (yrs) & $45 \pm 10$ & $42 \pm 10$ & $49 \pm 8$ & 0.203 \\
Height (cm) & $170 \pm 10.0$ & $168.5 \pm 8.4$ & $173 \pm 13$ & 0.464 \\
Mass (kg) & $73.0 \pm 11.6$ & $70.1 \pm 11.7$ & $78.6 \pm 10.4$ & 0.194 \\
BMI (kg/m²) & $25.2 \pm 3.5$ & $24.6 \pm 3.0$ & $26.6 \pm 4.4$ & 0.316 \\
Fat Free Mass (kg) & $49.8 \pm 9.0$ & $48.8 \pm 9.5$ & $51.7 \pm 8.5$ & 0.568 \\
Fat Mass (kg) & $21.9 \pm 6.4$ & $20.4 \pm 5.3$ & $25.0 \pm 8.0$ & 0.199 \\
Body Fat $(\%)$ & $29.5 \pm 6.9$ & $28.5 \pm 6.4$ & $31.4 \pm 8.2$ & 0.459 \\
VNS output current (mA) & $1.58 \pm 0.55$ & $1.55 \pm 0.59$ & $1.65 \pm 0.52$ & 0.752 \\
VNS implant time (months) & $59 \pm 19$ & $64 \pm 15$ & $50 \pm 24$ & 0.185 \\
VNS implant mass $(\mathrm{kg})$ & $71.2 \pm 12.5$ & $69.8 \pm 13.9$ & $72.8 \pm 12.0$ & 0.717 \\
VNS implant BMI (kg/m²) & $24.7 \pm 3.4$ & $24.9 \pm 3.1$ & $24.5 \pm 4.1$ & 0.861
\end{tabular}


Table 3. Energy expenditure, core and skin temperatures, skin perfusion and Standard Uptake Values (SUV Mean) for Brown Adipose Tissue (BAT), all muscles analyzed (Muscle), deltoid muscle (Deltoid), biceps muscle (Biceps), triceps muscle (Triceps), erector spinae muscle at the level of vertebrae C7 (C7), T8 (T8), L3 (L3), subcutaneous white adipose tissue (WAT Sc), visceral white adipose tissue (WAT Visc), the liver (Liver) and the brain at the level of the cerebellum (Brain). AU denotes Arbitrary Units for skin perfusion. ${ }^{\star} \mathrm{P}<0.05,{ }^{* *} \mathrm{P}<0.001$

\begin{tabular}{lccccc} 
Parameter & On & Off & P-value & TN & P-value \\
\hline Energy expenditure $(\mathrm{J} / \mathrm{s})$ & $68.6 \pm 7.9$ & $67.2 \pm 8.1$ & $0.038^{*}$ & $68.2 \pm 1.5$ & 0.107 \\
Core temperature $\left({ }^{\circ} \mathrm{C}\right)$ & $37.0 \pm 0.3$ & $36.9 \pm 0.4$ & 0.738 & $36.9 \pm 0.2$ & 0.877 \\
Mean skin temperature $\left({ }^{\circ} \mathrm{C}\right)$ & $33.5 \pm 0.8$ & $33.6 \pm 0.7$ & 0.686 & $33.2 \pm 0.4$ & $<0.001^{* *}$ \\
Proximal skin temperature $\left({ }^{\circ} \mathrm{C}\right)$ & $34.6 \pm 1.1$ & $34.6 \pm 0.5$ & 0.996 & $34.0 \pm 1.3$ & $0.012^{*}$ \\
Distal skin temperature $\left({ }^{\circ} \mathrm{C}\right)$ & $31.7 \pm 1.6$ & $33.0 \pm 1.7$ & $0.016^{*}$ & $31.7 \pm 2.5$ & $<0.001^{*}$ \\
Perfusion Foot $(\mathrm{AU})$ & $8.2 \pm 3.9$ & $13.4 \pm 21.4$ & 0.396 & $9.6 \pm 8.0$ & 0.553 \\
Perfusion Hand $(\mathrm{AU})$ & $54.5 \pm 35.3$ & $81.7 \pm 70.5$ & 0.149 & $25.1 \pm 11.4$ & $0.029^{*}$ \\
SUVMean BAT & $0.55 \pm 0.25$ & $0.67 \pm 0.46$ & 0.619 & $0.65 \pm 0.29$ & $0.012^{*}$ \\
SUVMean Muscle & $0.57 \pm 0.14$ & $0.65 \pm 0.11$ & 0.100 & $0.78 \pm 0.14$ & $0.012^{*}$ \\
SUVMean Deltoid & $0.57 \pm 0.16$ & $0.62 \pm 0.10$ & 0.281 & $0.74 \pm 0.11$ & $0.031^{*}$ \\
SUVMean Biceps & $0.62 \pm 0.17$ & $0.69 \pm 0.17$ & 0.390 & $0.89 \pm 0.14$ & 0.130 \\
SUVMean Triceps & $0.51 \pm 0.14$ & $0.61 \pm 0.13$ & $0.024^{*}$ & $0.70 \pm 0.16$ & 0.260 \\
SUVMean C7 & $0.62 \pm 0.22$ & $0.66 \pm 0.13$ & 0.572 & $0.85 \pm 0.23$ & 0.209 \\
SUVMean T8 & $0.63 \pm 0.12$ & $0.72 \pm 0.10$ & 0.081 & $0.79 \pm 0.16$ & 0.475 \\
SUVMean L3 & $0.46 \pm 0.15$ & $0.60 \pm 0.16$ & 0.057 & $0.75 \pm 0.29$ & 0.389 \\
SUVMean WAT SC & $0.19 \pm 0.09$ & $0.24 \pm 0.08$ & 0.194 & $0.24 \pm 0.09$ & 0.742 \\
SUVMean WAT VisC & $0.36 \pm 0.16$ & $0.55 \pm 0.30$ & 0.111 & $0.47 \pm 0.13$ & 0.881 \\
SUVMean Liver & $1.60 \pm 0.46$ & $2.04 \pm 0.45$ & $0.038^{*}$ & $2.44 \pm 0.41$ & 0.405 \\
SUVMean Brain & $6.58 \pm 2.07$ & $6.82 \pm 1.65$ & 0.730 & $7.45 \pm 1.68$ & 0.183
\end{tabular}

3). However, for all muscles together there was no significant change in activity (Muscle SUVMean; $0.57 \pm 0.14$ versus $0.65 \pm 0.11, P=0.100$, Table 3 ). The FDG-uptake in the liver significantly increased during VNS (Liver SUVMean; $1.60 \pm 0.46$ versus $2.04 \pm 0.45, P=0.038$, Table 3). After cold exposure, all subjects showed increased BAT activity (BAT SUVMean; $0.65 \pm 0.29$ versus $3.40 \pm 1.63, P=0.012$, Table 3, Figure 3). Deltoid muscle activity was significantly decreased in the cold (Deltoid SUVMean; 
Figure 1. Basal metabolic rate (BMR) during active and inactive VNS in relation to BAT activity. A. Individual responses upon VNS intervention. Notice all but one (marked line) subjects decreased energy expenditure upon deactivation of the VNS (subject that increased energy expenditure also described in Results section). B. Mean group BMR upon VNS intervention. C. The change in energy expenditure ( $\Delta$ Energy Expenditure) upon VNS intervention (On/Off) correlated to the change in BAT activity. One subject (open dot) showed a high increase in energy expenditure upon VNS intervention. This subject was confirmed as an statistical outlier in the exponential growth equation shown and therefore excluded from the equation. Values shown are means+S.E.M. Significance shown for paired t-test in VNS intervention group consisting of 4 males and six females. ${ }^{\star} \mathrm{P}<0.05$
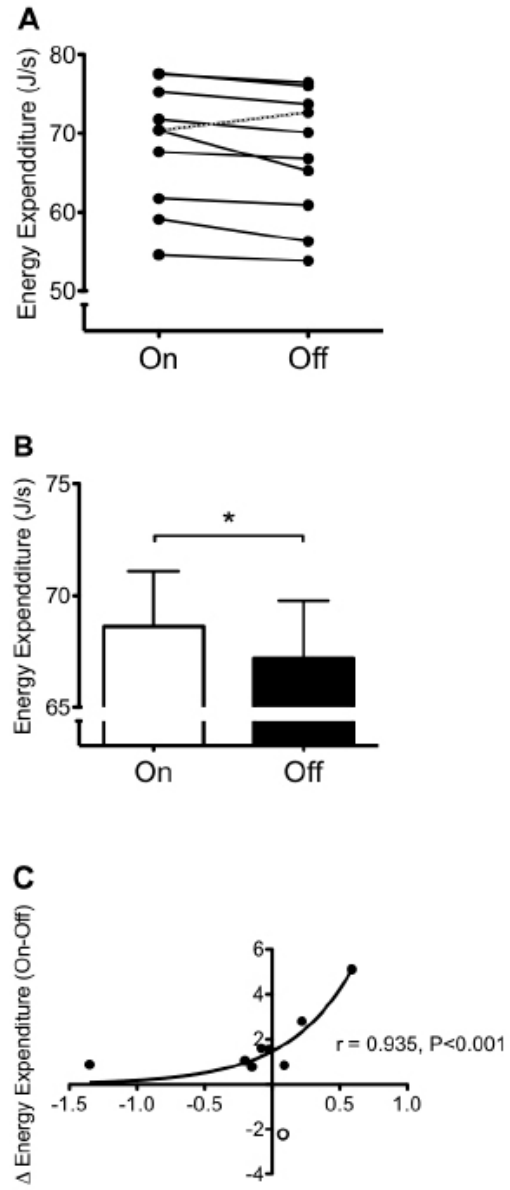

$\triangle$ BAT (On-Off)

$0,74 \pm 0.11$ versus $0.65 \pm 0.14, P=0.031$, Table 3 , Figure 3 ) and also activity for all muscles together decreased upon cold exposure (Muscle SUVMean; $0.78 \pm 0.14$ versus $0.72 \pm 0.14, P=0.012$, Table 3, Figure 3).

\section{Correlations}

Energy expenditure during VNS-On and VNS-Off measurements was not related to BAT activity, activity of other tissue (Muscle, WAT), skin perfusion, core and skin temperatures or any other study parameter. However, the change in energy expenditure upon VNS intervention (from VNS-On to VNS-Off) was positively 


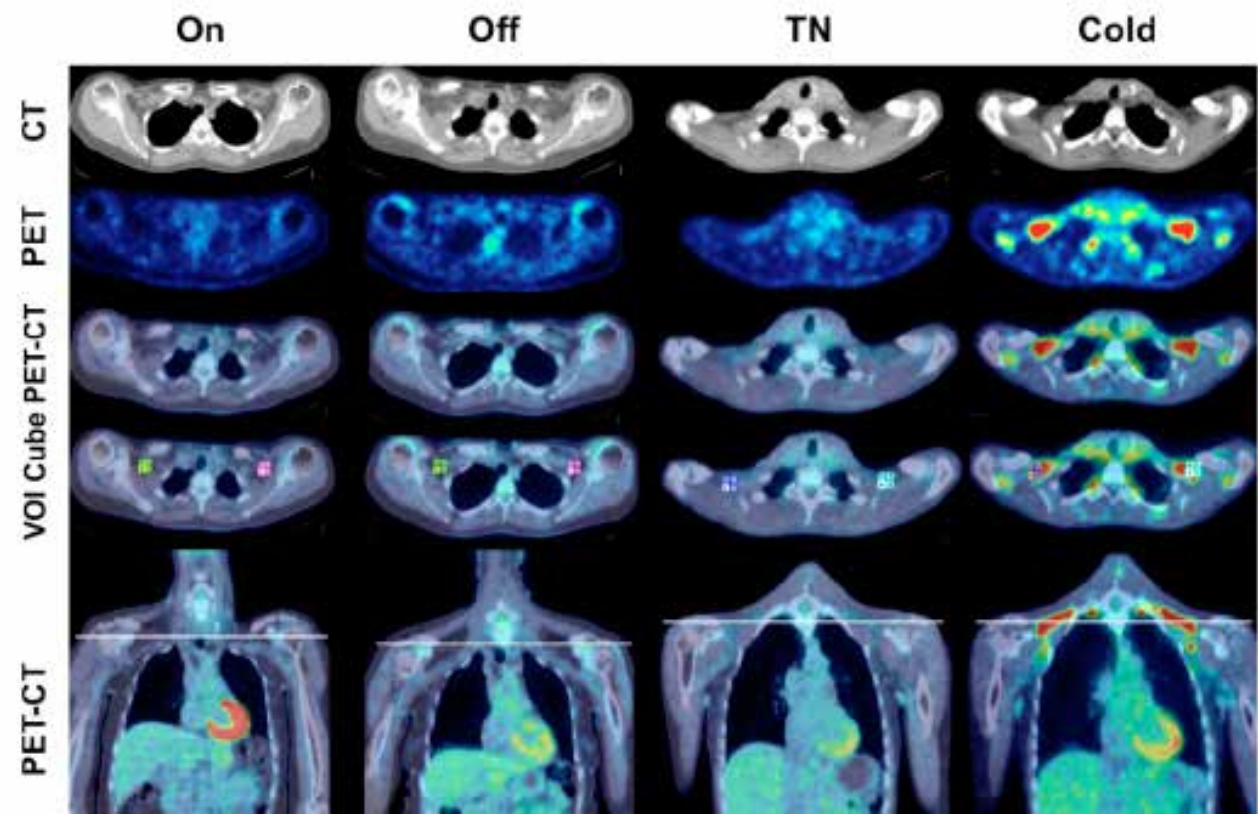

Figure 2. FDG-PET-CT images of intervention group and cold exposed subjects. Transaxial and coronal CT, PET and PET-CT fused images of VNS-On and VNS-Off from a subject in the VNS-On/Off group and images from a subject in the VNS-TN/Cold group during thermoneutral conditions (TN) and cold exposure (Cold). PET-CT image shown with and without Volume-Of-Interest (VOI) cubes for determination of activity. The white line in the coronal image indicates the transaxial slice shown above.

correlated to the change in BAT activity (exponential curve fitting, $r=0.935$, $\mathrm{P}<0.001$, Figure 1). VNS output current was not related to BAT activity or energy expenditure.

\section{Discussion}

This study shows that even short-term intervention of VNS therapy by turning off the VNS for only several hours significantly decreased energy expenditure in a cohort of treatment-stable VNS patients. In addition, the change in energy expenditure upon inactivation of VNS was significantly related to a change in BAT activity. Therefore, VNS possibly positively influences energy balance by increasing energy expenditure through involvement of BAT in humans. This might induce weight loss and as such VNS might contribute to weight management.

\section{Comparison with previous studies on VNS therapy}

An effect of VNS on metabolism had already been suggested by the authors of a 


\section{A}

8.07
4.01

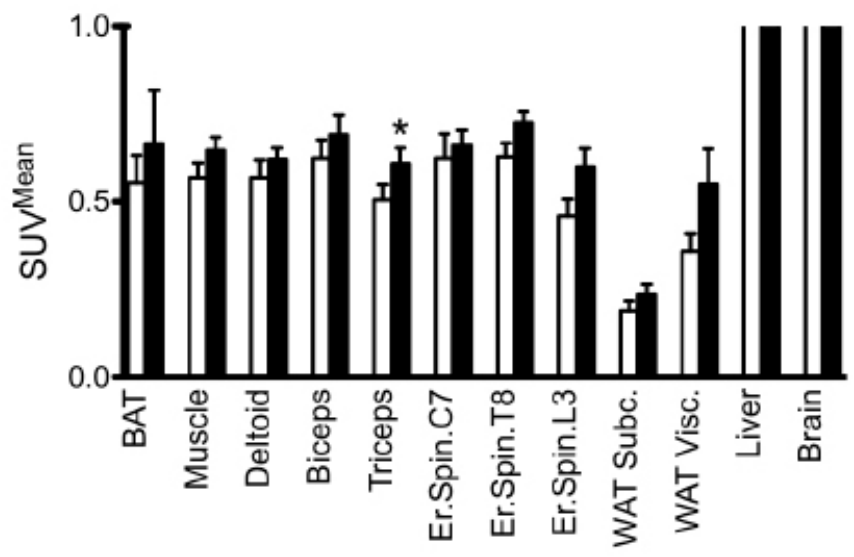

B

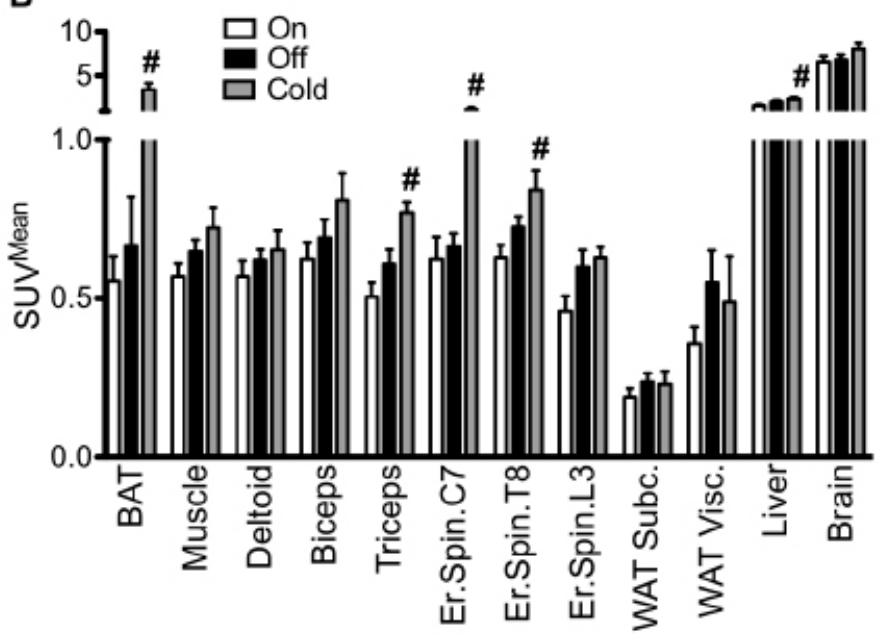

Figure 3. FDG-PET-CT activity of different tissue types upon VNS intervention. SUVMean (Standard Uptake Value)w values for Brown Adipose Tissue (BAT), all muscle (Muscle), deltoid muscle (Deltoid), biceps muscle (Biceps), triceps muscle (Triceps), erector spinae muscle at the level of vertebrae C7 (C7), T8 (T8), L3 (L3), subcutaneous white adipose tissue (WAT Sc), visceral white adipose tissue (WAT Visc), the liver (Liver) and the brain at the level of the cerebellum (Brain). A. Comparison of activity during VNS-On and VNS-Off. B. Comparison of activity between VNS-On, VNS-Off and VNS-Cold. Values shown are mean+S.E.M. ${ }^{*} \mathrm{P}<0.05$ for paired $\mathrm{t}$-tests between VNS-On and VNS-Off. $\# \mathrm{P}<0.05$ for one-way ANOVA with post-hoc Bonferroni correction between VNS-On, VNS-Off and VNS-Cold. 
brief report where weight loss was observed in 17/27 patients with VNS for epilepsy (2002). ${ }^{17}$ Pardo et al. studied a morbidly obese cohort of 14 VNS patients treated for depression (weight $91 \pm 27 \mathrm{~kg}, \mathrm{BMI} 43 \pm 5 \mathrm{~kg} / \mathrm{m}^{2}$ ) with VNS settings comparable to the present study ( $>24$ months VNS implant, Output current; 0.25-1.5 mA, Freq; $30 \mathrm{~Hz}$, Pulse; 500- $\mathrm{s}$, On-period; 30s, Off-period; 5 min, VNS current study; Output current; $1.58 \pm 0.55 \mathrm{~mA}$, Freq; $30 \pm 0 \mathrm{~Hz}$, Pulse; $266.7 \pm 64.5 \mu \mathrm{s}$, On-period; 23.9 \pm 10.5 s, Off-period; $3.7 \pm 2.1 \mathrm{~min}$ ) and observed a significant BMI-related weight loss after VNS therapy. ${ }^{16}$ The short-term intervention in normal weight subjects in the current study already showed a significantly increased energy expenditure during VNS. Although the mean difference in BMR was small, the weight loss observed by Pardo et al. in morbidly obese subjects suggests long-term VNS results in increased energy expenditure and could be important in weight maintenance.

Another study reported 33 obese patients (BMI; $30.1 \pm 3.6 \mathrm{~kg} / \mathrm{m}^{2}$ ) receiving VNS for depression and investigated the response to a food stimulus upon active and inactive VNS. ${ }^{19}$ Short-term VNS intervention significantly altered food craving. Duration of VNS intervention was not specified, but seems to have taken place within several hours, which is similar to the present study. ${ }^{19}$

All currently published data on the effect of VNS on metabolism in humans are retrospective. However, in animals a prospective study in overfed minipigs undergoing bilateral VNS (implanted $5 \mathrm{~cm}$ above the diaphragm) with stimulation parameters comparable to our study (VNS minipigs; Output current; 2mA, Freq; $30 \mathrm{~Hz}$, Pulse; 500- $\mu \mathrm{s}$, On-period; 30 s, Off-period; 5 min, VNS current study; see above) has revealed that weight gain was prevented in the VNS-treated minipigs and not in controls. ${ }^{24}$ VNS in these minipigs significantly decreased food intake, especially carbohydrate consumption ('sweet craving') as assessed by the respiratory quotient (RQ). ${ }^{24}$ In the present study we did not observe a difference in RQ after VNS intervention (RQ On; $0.82 \pm 0.03$ versus RQ Off; $0.82 \pm 0.04, P=0.8774$ ) and the mean $\mathrm{RQ}$ of \pm 0.8 confirms the prescribed overnight fast preceeding the $\mathrm{BMR}$ measurments. However, possibly $R Q$ does show a significant difference in man when studying VNS in longer time periods, for example before and after VNS implantation.

Finally, in a study on the effect of VNS on metabolism Sobocki et al. used a crossover design in pigs with activation and de-activation, each in a 4-week period and found that VNS prevented weight gain but did not affect metabolic rate as assessed by indirect calorimetry. ${ }^{25}$ However, metabolic rate was determined under general anesthesia, which probably affected BMR measurements. Possibly BMR did change under awake conditions, as was observed in the current report, which 
would explain the significant differences in weight gain observed by Sobocki et al.

\section{Mechanism of VNS increased energy expenditure}

Possible mechanisms by which VNS affects energy expenditure have been suggested in several studies. From an anatomical viewpoint, it is interesting to note that the majority of fibers present within the vagus nerve are afferent fibres (74\%) and only a minority (26\%) are efferent, indicating that the vagus nerve is both an afferent nerve and efferent nerve as well. ${ }^{26}$

Within the hypothalamus, several areas have been studied in respect to the regulation of energy balance. The hypothalamic arcuate nucleus receives vagus signals and projects them to the paraventricular nucleus (PVN), which in turn signals to the sympathetic nervous system and to the thyroid axis. ${ }^{27}$ Vagotomy blocks these responses and alters energy homeostasis. ${ }^{27}$ In the ventromedial nucleus of the hypothalamus $(\mathrm{VMH})$, an area adjacent to the PVN, both parasympathetic and sympathetic neurons are present, and it is suggested the $\mathrm{VMH}$ regulates the 'balance' between sympathetic and parasympathetic nerve activities. Therefore, $\mathrm{VMH}$ stimulation may affect sympathetic mechanisms. Electrical stimulation of the $\mathrm{VMH}$ increased energy expenditure and decreased $\mathrm{RQ}$ in rats, suggesting an increased fat oxidation. ${ }^{28}$ Indeed, $\mathrm{VMH}$ stimulation, but not PVN-stimulation, increases norepinephrine turnover and thermogenesis in interscapular BAT in rats, and this effect is completely inhibited by blockage of sympathetic ganglions. ${ }^{29,30}$ Interestingly, vagotomy has been demonstrated to decrease NE-turnover in BAT, ${ }^{11}$ suggesting that vagus signals may influence BAT thermogenesis. In the present study, we observed a significant change in energy expenditure that was related to changes in the activity in BAT and not to changes in any of the other tissues that we have measured. Despite the fact that mean BAT activity did not increase upon VNS, the change in BAT activity explained a large part of the change in energy expenditure. To our opinion, this suggests at least part of the effect of VNS intervention on energy expenditure can be explained by BAT activity.

\section{BAT presence and activity in refractory epilepsy}

Since BAT activity is reported to decrease with age ${ }^{31}$ and can be affected by several pathophysiological conditions, ${ }^{32}$ we considered the possibility of reduced presence of BAT in (these) patients with refractory epilepsy. Therefore, we investigated maximal BAT activity in VNS patients after mild cold exposure. Mild cold exposure is known to induce BAT activation in $100 \%$ of lean young subjects (age; $24.3 \pm 3.6$ years, BMI; 
$23.2 \pm 1.2 \mathrm{~kg} / \mathrm{m}^{2}$ ) and to be related to $B M R$ and cold-induced thermogenesis. $3,5,6$ Despite their relatively high mean age ( $49 \pm 8$ years) but comparable BMI $(26.6 \pm 4.4$ $\mathrm{kg} / \mathrm{m}^{2}$ ), cold-induced BAT activity was present in all five patients. After mild cold stimulation BAT presence and activity were significantly higher than under TN conditions (VNS-Cold; BAT SUVMean $3.40 \pm 1.63$ versus VNS-TN; BAT SUVMean $0.65 \pm 0.29, P=0.012)$, but slightly lower as compared to previously studied healthy young subjects (Age; $23 \pm 2$ years, BMI; $23.1 \pm 1.7 \mathrm{~kg} / \mathrm{m}^{2}$, BAT SUVMean; $7.19 \pm 2.09$ versus current study BAT SUVMean; $3.40 \pm 1.63, P=0.010$, Vosselman et al., unpublished data). We believe this may be attributed to an age-related decrease in BAT activity. ${ }^{31}$ Therefore, BAT thus does not appear to be decreased in patients with refractory epilepsy.

\section{Effect of VNS on muscle and liver metabolism}

In contrast to the significant increase in energy expenditure that was related to BAT activity, we observed a decrease in liver and triceps muscle glucose uptake on FDG-PET-CT upon VNS. This is in line with the observation that in rodents cannabinoid receptor 1 (CB1) antagonism is known to induce sympathetic nervous system (SNS) activity and increase energy expenditure and BAT activity (as measured by the temperature in BAT itself), but not liver and muscle activity. ${ }^{33}$ In addition, CB1 antagonism also induces fatty acid oxidation, ${ }^{34}$ which could be the effect of the increased BAT activation, since BAT was recently shown to be able to combust lipids. ${ }^{35}$ Moreover, it is known increased fatty acid oxidation can lead to inhibition of glucose uptake (the so-called Randle effect), ${ }^{36}$ which would decrease activity on FDG-PET-CT imaging since this selectively depicts glucose uptake. Therefore, although there is a decrease in FDG-PET-CT-activity in liver and muscle, there could still be increased metabolism upon VNS in these tissues resulting from increased fatty acid oxidation. This would be consistent with an increase in BAT fatty acid oxidation and increased energy expenditure upon VNS. However, in this study we were not able to study fatty acid metabolism since FDG-PET-CT is a glucose tracer. In this regard, more research focused on liver and muscle function as well as fatty acid metabolism upon VNS is needed. 


\section{Conclusion}

In summary, the present study demonstrates for the first time that VNS is accompanied by an increase in whole body energy expenditure, compared to short term deactivation of VNS. Moreover, the increase in energy expenditure was positively related to the change in BAT activity. This suggests that the VNS-induced increase in energy expenditure is at least in part due to changes in BAT activity. Therefore, chronic VNS might have a beneficial effect on energy balance and might play a role in weight management. However, the observed mean differences in energy expenditure are small, probably to a large extent because of the short duration of the intervention, and future, long-term, prospective studies should point out if VNS has a clinically and therapeutically significant effect on whole body metabolism. In addition, detailed metabolic imaging studies should assess the effect of VNS on BAT and other tissues in order to identify new possible targets in the treatment of obesity. 


\section{References}

1. Simpson SA, Shaw C, McNamara R. What is the most effective way to maintain weight loss in adults? BMJ 2011;343:d8042.

2. Cannon B, Nedergaard J. Brown adipose tissue: function and physiological significance. Physiol Rev 2004;84:277-359.

3. van Marken Lichtenbelt WD, Vanhommerig JW, Smulders NM, et al. Cold-activated brown adipose tissue in healthy men. N Engl J Med 2009;360:15008.

4. Virtanen KA, Lidell ME, Orava J, et al. Functional brown adipose tissue in healthy adults. N Engl J Med 2009;360:1518-25.

5. Vijgen GH, Bouvy ND, Teule GJ, Brans B, Schrauwen P, van Marken Lichtenbelt WD. Brown adipose tissue in morbidly obese subjects. Plos One 2011;6:e17247.

6. Yoneshiro T, Aita S, Matsushita M, et al. Brown Adipose Tissue, WholeBody Energy Expenditure, and Thermogenesis in Healthy Adult Men. Obesity (Silver Spring) 2011;19:13-6.

7. Fruhbeck G, Becerril S, Sainz N, Garrastachu P, Garcia-Velloso MJ. BAT: a new target for human obesity? Trends Pharmacol Sci 2009;30:387-96.

8. Zingaretti MC, Crosta F, Vitali A, et al. The presence of UCP1 demonstrates that metabolically active adipose tissue in the neck of adult humans truly represents brown adipose tissue. FASEB J 2009;23:3113-20.

9. Arch JR, Ainsworth AT, Cawthorne MA, et al. Atypical beta-adrenoceptor on brown adipocytes as target for anti-obesity drugs. Nature 1984;309:163-5.

10. Mano-Otagiri A, Iwasaki-Sekino A, Nemoto T, et al. Genetic suppression of ghrelin receptors activates brown adipocyte function and decreases fat storage in rats. Regul Pept;160:81-90.

11. Mano-Otagiri A, Ohata H, Iwasaki-Sekino A, Nemoto T, Shibasaki T. Ghrelin suppresses noradrenaline release in the brown adipose tissue of rats. J Endocrinol 2009;201:341-9.

12. Dedeurwaerdere S, Gilby K, Vonck K, Delbeke J, Boon P, Mclntyre D. Vagus nerve stimulation does not affect spatial memory in fast rats, but has both anti-convulsive and pro-convulsive effects on amygdala-kindled seizures. Neuroscience 2006;140:1443-51.

13. Laskiewicz J, Krolczyk G, Zurowski G, Sobocki J, Matyja A, Thor PJ. Effects of vagal neuromodulation and vagotomy on control of food intake and body weight in rats. J Physiol Pharmacol 2003;54:603-10. 
14. Kosel M, Schlaepfer TE. Beyond the treatment of epilepsy: new applications of vagus nerve stimulation in psychiatry. CNS Spectr 2003;8:515-21.

15. Privitera MD, Welty TE, Ficker DM, Welge J. Vagus nerve stimulation for partial seizures. Cochrane Database Syst Rev 2002:CD002896.

16. Pardo JV, Sheikh SA, Kuskowski MA, et al. Weight loss during chronic, cervical vagus nerve stimulation in depressed patients with obesity: an observation. Int J Obes (Lond) 2007;31:1756-9.

17. Burneo JG, Faught E, Knowlton R, Morawetz R, Kuzniecky R. Weight loss associated with vagus nerve stimulation. Neurology 2002;59:463-4.

18. Bodenlos JS, Kose S, Borckardt JJ, et al. Vagus nerve stimulation and emotional responses to food among depressed patients. J Diabetes Sci Technol 2007;1:771-9.

19. Bodenlos JS, Kose S, Borckardt JJ, et al. Vagus nerve stimulation acutely alters food craving in adults with depression. Appetite 2007;48:145-53.

20. Bligh J, Johnson KG. Glossary of terms for thermal physiology. J Appl Physiol 1973;35:941-61.

21. van Marken Lichtenbelt WD, Daanen HA, Wouters L, et al. Evaluation of wireless determination of skin temperature using iButtons. Physiol Behav 2006;88:489-97.

22. Kingma B, Frijns A, van Marken Lichtenbelt W. The thermoneutral zone: implications for metabolic studies. Front Biosci (Elite Ed) 2012;4:1975-85.

23. Vijgen GH, Bouvy ND, Teule GJ, et al. Increase in Brown Adipose Tissue Activity after Weight Loss in Morbidly Obese Subjects. J Clin Endocrinol Metab 2012.

24. Val-Laillet D, Biraben A, Randuineau G, Malbert $\mathrm{CH}$. Chronic vagus nerve stimulation decreased weight gain, food consumption and sweet craving in adult obese minipigs. Appetite 2010;55:245-52.

25. Sobocki J, Fourtanier G, Estany J, Otal P. Does vagal nerve stimulation affect body composition and metabolism? Experimental study of a new potential technique in bariatric surgery. Surgery 2006;139:209-16.

26. Prechtl JC, Powley TL. The fiber composition of the abdominal vagus of the rat. Anat Embryol (Berl) 1990;181:101-15.

27. Murphy KG, Bloom SR. Gut hormones and the regulation of energy homeostasis. Nature 2006;444:854-9.

28. Ruffin $M$, Nicolaidis $S$. Electrical stimulation of the ventromedial hypothalamus enhances both fat utilization and metabolic rate that precede and parallel the inhibition of feeding behavior. Brain Res 1999;846:23-9. 
29. Saito $M$, Minokoshi $Y$, Shimazu T. Accelerated norepinephrine turnover in peripheral tissues after ventromedial hypothalamic stimulation in rats. Brain Res 1989;481:298-303.

30. Perkins MN, Rothwell NJ, Stock MJ, Stone TW. Activation of brown adipose tissue thermogenesis by the ventromedial hypothalamus. Nature 1981;289:401-2. 31. Saito $M$, Okamatsu-Ogura $Y$, Matsushita $M$, et al. High Incidence of Metabolically Active Brown Adipose Tissue in Healthy Adult Humans: Effects of Cold Exposure and Adiposity. Diabetes 2009;58:1526-31.

32. Vijgen $\mathrm{GH}$, van Marken Lichtenbelt W. Brown adipose tissue: clinical impact of a re-discovered thermogenic organ. Front Biosci 2012;Accepted for publication. 33. Bajzer $M$, Olivieri $M$, Haas $M K$, et al. Cannabinoid receptor 1 (CB1) antagonism enhances glucose utilisation and activates brown adipose tissue in diet-induced obese mice. Diabetologia 2011;54:3121-31.

34. Nogueiras R, Veyrat-Durebex C, Suchanek PM, et al. Peripheral, but not central, CB1 antagonism provides food intake-independent metabolic benefits in diet-induced obese rats. Diabetes 2008;57:2977-91.

35. Bartelt A, Bruns OT, Reimer R, et al. Brown adipose tissue activity controls triglyceride clearance. Nat Med 2011;17:200-5.

36. Randle PJ, Garland PB, Hales CN, Newsholme EA. The glucose fattyacid cycle. Its role in insulin sensitivity and the metabolic disturbances of diabetes mellitus. Lancet 1963;1:785-9. 


$$
\theta_{-1}
$$




\section{Chapter 8}

General Discussion 


\section{Introduction}

Heat production in brown adipose tissue (BAT) serves a vital function in small mammals and human newborns in order to prevent hypothermia upon cold exposure. ${ }^{1}$ Recently, BAT has also been shown to be present and active in adult humans. ${ }^{2-4}$ In this thesis, we studied the physiology of human BAT from a cellular level to effects on whole body metabolism. In man, BAT activity is negatively related to the degree of obesity and positively related to energy expenditure. ${ }^{2,4,5}$ Since obesity results from a disturbed energy balance, it is interesting to study the physiology of human BAT. To increase the understanding of the function of human BAT in obesity, in this thesis we investigated BAT activity under pathophysiological conditions in morbid obesity. Moreover, we investigated if human BAT can be stimulated upon electrical nerve stimulation. In this final chapter the main findings of this thesis will be discussed and interpreted. In addition, possible clinical implications and future directions of BAT in treating disease will be addressed.

\section{Measuring human BAT on a cellular level}

In Chapter 6 for the first time cellular oxygen consumption was determined in surgical biopsies taken from the adipose tissue region known to contain BAT in man. When we compared the cellular oxygen consumption in this adipose tissue depot to subcutaneous WAT, we strikingly found a $\sim 300 \%$ higher level of oxygen consumption in adipose tissue derived from the BAT region. These results thus confirmed the presence of metabolically active BAT in the neck. However, our measurements were performed under baseline conditions without the addition of stimuli to increase BAT activity as cold or sympathetic nervous stimuli. In pure brown adipocytes derived from mice it was possible to increase BAT activity $700-800 \%$ upon NE-infusion. ${ }^{6}$ Therefore, possibly the addition of adequate stimuli can even further increase human BAT activity. On top of that, adipose tissue from the BAT region does not solely consists of 'pure' brown adipocytes, but rather seems to be a mixture of several types of adipocytes that vary in size in mitochondrial content. ${ }^{7}$ To characterize these cells, it is important to understand the origin of BAT. Classically, it was thought that during development preadipocytes irreversibly differentiated into the brown or white phenotype, without being able to change throughout life. However, histological observations suggested there also were adipocytes that could 'turn into one another'. ${ }^{8}$ In 2007 it was shown that addition of rosiglitazone (an antidiabetic drug known to stimulate the transcription factor PPAR-gamma) to cultured WAT-precursors induced the development of 
UCP1-containing thermogenic cells. ${ }^{9}$ These cells were genetically different from BAT, but able to generate a similar level of thermogenesis, and therefore baptized BRITE (brown-in-white) adipocytes. At the same time, the discovery of PRDM16, a zinc-finger protein, gave more insight into the development of $\mathrm{BAT}^{10}$ and later it was shown PRDM16 and BMP7 are crucial factors in the development of BAT.11,12 Following the experiments tracing back the development of BAT, it was also shown that prostaglandins (more specific COX-2, which is interestingly inhibited by most anti-inflammatory drugs) were able to induce 'browning'.13,14 Moreover, preadipocytes capable of becoming thermogenic were also shown to reside in skeletal muscle and a specific exercise-induced myokine capable of 'browning' was discovered; irisin. ${ }^{15-17}$ In man, in cultured biopsies taken from the same adipose tissue region as described in Chapter 6, it was shown to be possible to reproduce the rosiglitazone-effect in vitro. ${ }^{18}$ In addition, molecular characterization was done on human adipose tissue biopsies from our center, similar to the biopsies studied in Chapter 6. The results showed the adipocytes present in these tissue depots could be characterized as 'beige' adipose tissue, and not BAT. ${ }^{19}$ Full-grown beige cells were shown to form stable adipose tissue depots after transplantation in mice. ${ }^{19}$ In the murine beige cells studied cellular respirometry was performed, similar to the method we used in Chapter 6. Murine beige cells showed a significantly higher baseline respiration and oligomycin-insensitive uncoupled respiration compared to WAT. ${ }^{19}$ Also, under unstimulated conditions, the contribution of uncoupled respiration to baseline respiration was $78 \%$ for BAT and $60 \%$ for beige cells, suggesting UCP1-induced mitochondrial uncoupling is already present without any stimulus. In Chapter 6 , in our biopsies from the human BAT region, 83\% of baseline respiration was derived from uncoupled respiration, which confirms findings observed in mammals. ${ }^{19}$ Unfortunately, we were not able to determine the effect of, for example, ß-adrenergic stimulation on human biopsies, which has been shown to dramatically increase cellular respiration up to $700-800 \%$ of baseline. ${ }^{6}$

\section{Measuring human BAT on a whole body level}

The development of new imaging techniques as FDG-PET-CT allowed for detailed study of metabolically active tissue activity and in a prospective study under controlled conditions of cold exposure from our lab FDG-PET-CT showed active BAT in lean young adults in 2009. ${ }^{2}$ Other reports that were published in the same period also showed consistent BAT activity in humans and therefore a renewed curiosity for investigating BAT in man was born. 3,4,20 In this thesis, in Chapter $\mathbf{3}$ we 
found that - in combination with our group of previously studied lean subjects ${ }^{2}$ BAT activity was robustly related to BMI and percentage body fat. This suggests a sufficient level of BAT activity could play an important role in maintaining body weight. Chronic overfeeding previously showed large interindividual differences in weight gain, which were suggested to possibly result from undetermined genetic factors. ${ }^{21}$ Interestingly, genetic polymorphisms in the UCP1-gene have been shown to be related to the energy balance (Chapter 2). Moreover, recently it was shown that polymorphisms of the UCP1-gene and the gene for the $\beta 3$-adrenergic receptor, both essential for BAT thermogenesis (Chapter 2), may be associated with visceral fat accumulation during life. ${ }^{22}$ Therefore, genetic factors predisposing for low BAT activity could possibly contribute to the development of obesity.

Intermezzo

\section{Measuring human BAT using an individualized cooling protocol}

From a methodological point of view, it is important to note that in Chapter 3 and 4 all morbidly obese subjects underwent an individualized cooling protocol in order to induce maximal NST. To adequately measure BAT activity using FDG-PET-CT imaging presently only cold exposure is known to be able to consistently promote FDG-uptake in BAT. However, the cold exposure needs to be strong enough to induce maximal NST, but low enough to prevent ST. This is necessary, since shivering in skeletal muscle would increase the muscular FDG-uptake and possibly decrease uptake in BAT resulting in an inadequate interpretation of BAT activity. Most dedicated studies on BAT activity use a standardized cooling level for each studied subject, not taking into account the interindividual differences in body composition and thermoneutral zone. ${ }^{23}$ When exposed to a similar level of cold exposure, a morbidly obese subject will experience a less intensive cold stimulus compared to a lean subject through their difference in subcutaneous adipose tissue insulation. ${ }^{24}$ Therefore, we cooled each subject until shivering occurred. Subsequently, we gradually increased temperature until shivering just stopped (Figure 1). This protocol ensures a maximal level of NST, and thus a maximal level of BAT activity for each subject, independent of body composition. 


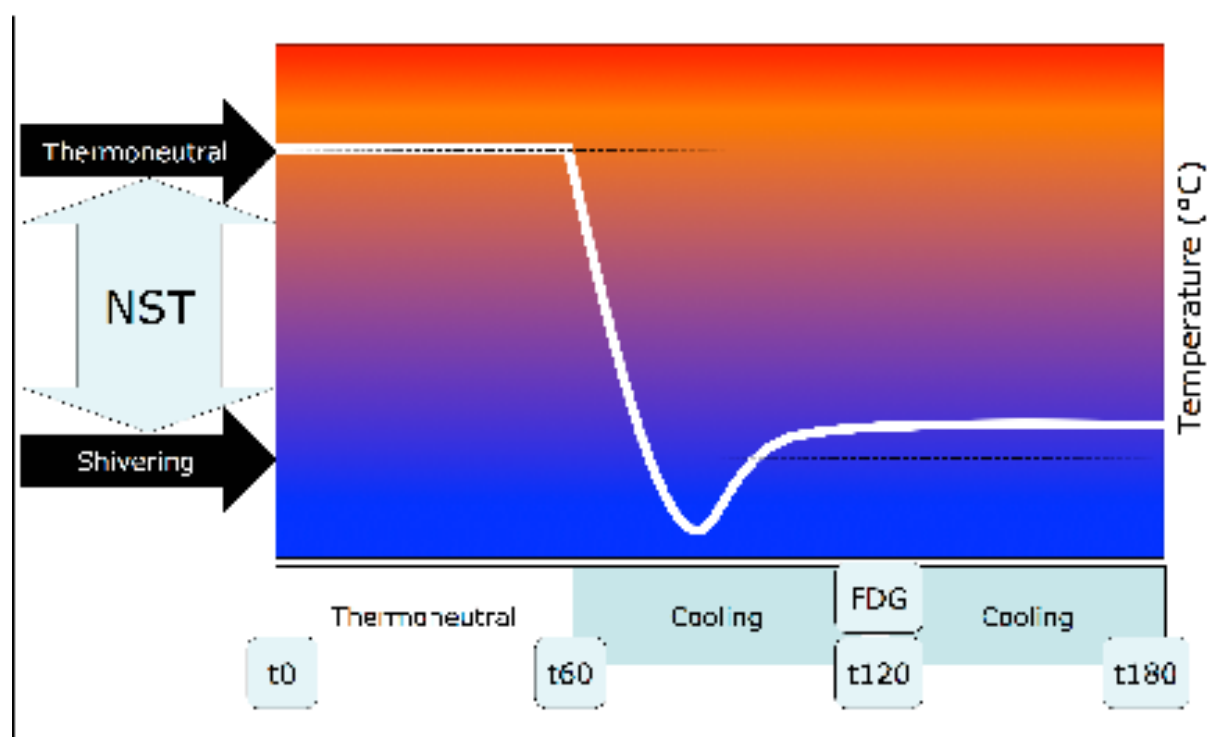

Figure 1. Individualized cooling protocol. During two hours of cooling maximal non-shivering thermogenesis (NST) is induced to stimulate brown adipose tissue (BAT) activity. Temperatures shown are room temperatures in degrees Celsius.

In Chapter 4, we observed an increase in BAT activity after a $\sim 35 \mathrm{~kg}$ weight loss. In addition, subjects with absent BAT activity during morbid obesity showed recruitment of BAT activity upon weight loss in regions that contained BAT activity in the lean subjects studied before. These results suggest BAT is rather a consequence than a cause of obesity. After observing an increase in BAT activity after weight loss in Chapter 4, we also studied the effect of weight loss on skeletal muscle mitochondrial uncoupling in Chapter 5. Although we did not observe a change in skeletal muscle mitochondrial uncoupling, the ADP-stimulated skeletal muscle metabolism did significantly increase after weight loss. This suggests that in addition to 'restoring' the level of BAT activity, sufficient weight loss is also able to recover obesity-associated impaired skeletal muscle metabolism. Although we were unable to define the molecular mechanism responsible for the observed recruitment of BAT, the observed BAT recruitment provides hopeful evidence for future studies targeting BAT recruitment in man. Moreover, as discussed in Chapter $\mathbf{2}$, in addition to obesity other studies have now shown relations between a low BAT 
activity and type 2 diabetes, hepatosteatosis and hypothyroidism, which even more emphasizes that BAT seems to be play a relevant role in both human physiology and pathophysiology.

Intermezzo

\section{Functional imaging of human BAT}

The first evidence of functional BAT in humans was shown using FDG-PET-CT imaging studies (Figure 2). ${ }^{25,26}$ Prospective studies using controlled cold exposure to maximize NST in BAT showed high levels of BAT activity which was related to body composition and energy expenditure. ${ }^{2-4}$ However, FDG-PET-CT relies on the concept of cellular uptake of radioactive glucose (FDG). Interestingly, detailed studies in the mammalian brown adipocyte have shown the main fuel for BAT are free fatty acids (FFAs). ${ }^{1}$ Glucose uptake in BAT is considered low ( 10\%) and is suggested to function in order to maintain the mitochondrial membrane proton gradient or to be part of the glycerol component of FFAs (Chapter 2). Estimations made from glucose clearance rates in dynamic FDG-PET-CT studies, assuming glucose contributes for $10 \%$ and FFAs $90 \%$ to BAT metabolism, suggest a metabolic contribution for BAT of $\sim 5 \%$ to the basal metabolic rate. ${ }^{27}$ However, this is a rough estimation since FFA-metabolism was not quantified and most

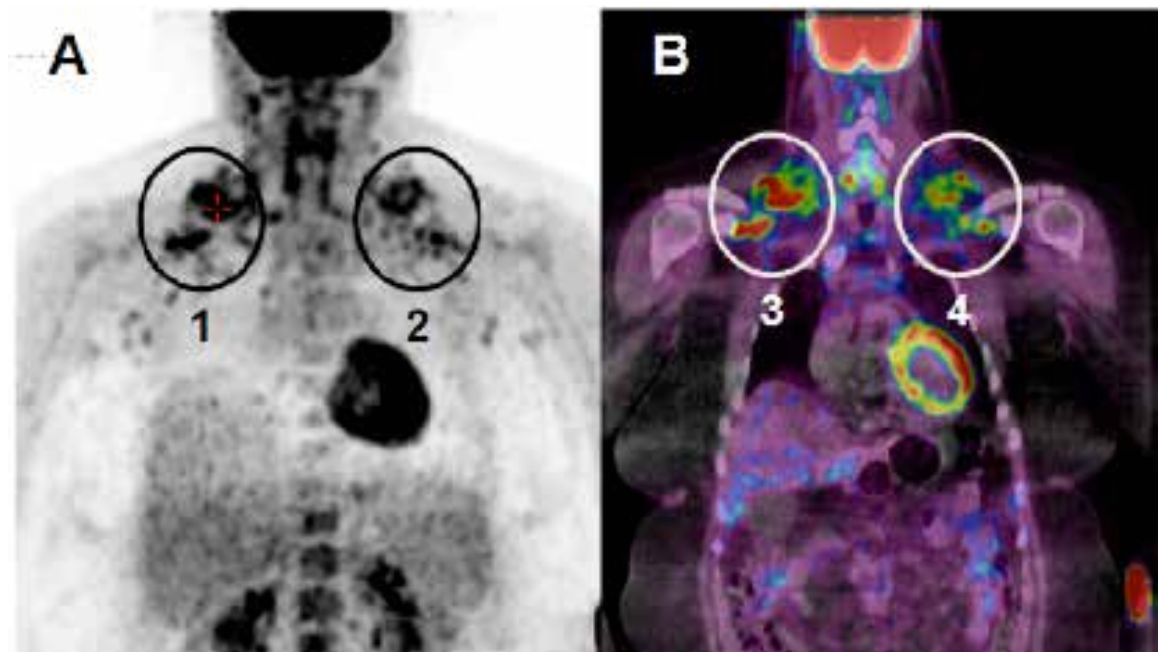

Figure 2. FDG-PET-CT-scan of morbidly obese subject. The PET-image (A) shows FDG uptake supraclavicular on the right (1) and left (2) side. The combined PET-CT (B) shows activity in adipose tissue (3 and 4), confirming this is BAT. 
FFA-lipolysis in BAT is performed intracellular. ${ }^{1}$ Therefore, although FDG-PET-CT has facilitated the discovery of functional BAT in man, ideally one would like to use other metabolic tracers to determine the metabolic capacity of BAT. Interestingly, fatty acid uptake in human BAT was recently shown by using the fatty acid tracer ${ }^{18} \mathrm{FTHA} .{ }^{28}$ During cold exposure, fatty acid uptake in BAT was significantly higher compared to WAT. The increase in energy expenditure in this report was high (up to $80 \%$ ), but possibly resulted to a large extent from skeletal muscle shivering due to the strong cold exposure in this study. Another study showed a significant relation between BAT perfusion (as measured by radioactive labelled water $\left(\left({ }^{15} \mathrm{O}\right)\right.$ $\mathrm{H}_{2} \mathrm{O}$ ) and whole-body energy expenditure, ${ }^{29}$ confirming the relation found using $F D G{ }^{2}$ but unfortunately did not allow for determination of the contribution of BAT to NST. The most recent dedicated report measured oxygen consumption in BAT during cold exposure by dynamic oxygen $\left({ }^{15} \mathrm{O}\right)$ PET imaging, a possibly very adequate marker for BAT metabolism. ${ }^{30}$ Remarkably, the authors conclude the contribution of BAT is only $\sim 1 \%$ of the basal metabolic rate, much lower than reported above ( $5 \%$ ). However, the cooling period in this study was very short (20 min), whereas previously it was shown maximal NST is not generated until at least 60-80 minutes of cold exposure. ${ }^{31}$ In summary, no robust estimation of the contribution of BAT to the human energy balance has been shown yet, but prospective studies using adequate tracers and cooling methods can potentially determine the metabolic value of BAT in man.

\section{Stimulating BAT in man}

The above-discussed results suggest human BAT recruitment is possible and future studies should point out the effect of stimulating BAT differentiation in-vivo and implanting in-vitro developed tissue grafts. At this moment the most powerful and reproducible physiological stimulator of BAT is cold exposure, which increases sympathetic nervous system activity that is suggested to $ß$-adrenergically induce mitochondrial uncoupling in BAT (Chapter 2). In 1985, it was found that the ß-agonist ephedrine could not induce BAT activity in humans. ${ }^{32,33}$ However, the available methods for the assessment of BAT activity were limited to measuring tissue temperature with thermoprobes and therefore this experiment was recently repeated in combination with FDG-PET-CT studies. ${ }^{34}$ Although increased heart rate, blood pressure and energy expenditure showed the SNS was already clearly activated by a relatively high dose of ephedrine $(1.0 \mathrm{mg} / \mathrm{kg})$, BAT remained inactive in subjects that possessed active BAT upon cold exposure. ${ }^{34}$ A comparable study 
using isoprenaline to induce $ß$-adrenergic activation also did not show BAT activity. ${ }^{35}$ However, a high level of systemic ephedrine infusion $(2.5 \mathrm{mg} / \mathrm{kg})$ indeed induced BAT activity in lean but not obese subjects. ${ }^{36}$ Nonetheless, the BAT activity was low compared to cold-induced BAT activity and the high heart rate ( 75 BPM) and increased blood pressure $(\sim 160 \mathrm{~mm} / \mathrm{Hg})$ that are accompanied with such high levels of ephedrine should not be underestimated. Therefore, possibly mimicking the effect of cold exposure on BAT could be performed by direct peripheral or central nervous stimulation (CNS). Interestingly, in 1989 electrical stimulation of the ventromedial hypothalamus $(\mathrm{VMH})$ in the CNS of rats showed increased norepinephrine (NE) turnover in BAT in rats. ${ }^{37}$ This suggests electrical stimulus could possibly induce BAT activity in man. In practice, electrical stimulation of the CNS by vagus nerve stimulation (VNS) is performed in refractory epilepsy patients. ${ }^{38}$ VNS decreases seizure frequency, possibly due to alterations in central nervous system activity. ${ }^{38}$ However, the exact mechanism of action of VNS is not known yet. Interestingly, VNS treatment has been reported to be associated with weight loss. ${ }^{39,40}$ Animal studies have also shown significant weight loss, associated with a decreased food intake. ${ }^{41-43}$ Based on this literature, in Chapter 7 we investigated the effect of VNS on energy expenditure and BAT activity in a treatment stable cohort of refractory epilepsy patients. Strikingly, we found that a short-term deactivation ( 4 hours) already significantly decreased energy expenditure, which suggests VNS indeed increases energy expenditure and thus promote weight loss. On top of that, we observed a relation between the change in energy expenditure upon VNS and the change in BAT activity as observed by FDG-PET-CT. Although mean BAT activity did not change significantly upon the short period of deactivation, this shows that the increase in energy expenditure upon VNS could be facilitated by changes in BAT activity. All studied patients with refractory epilepsy showed increased BAT activity upon cold exposure, suggesting potentially high levels of active BAT could be induced by VNS. Therefore, the results described in Chapter 7 justify a prospective study that observes VNS before and after implantation to unravel the long-term effect of VNS on BAT activity. Subsequently, the effect of VNS could be studied in diseases other than refractory epilepsy associated with low BAT activity. In addition, possibly direct sympathetic stimulation could provide a more direct induction of BAT activity. However, electrical sympathetic nervous system stimulation has never been performed and animal studies should be done first. In addition, other mechanisms to alter BAT activity should be studied, since VNS is an invasive surgical procedure with unknown long-term effects. 


\section{Does BAT matter?}

In the sections above, we discussed the high metabolism of human BAT under ex-vivo conditions and the large differences in human BAT activity in vivo in the context of the energy balance. On top of that, this thesis showed VNS increased energy expenditure, which could partly be explained by changes in BAT activity. Together, these results suggest human BAT could be of significant physiological value. To be able to estimate the actual contribution of human BAT to human energy expenditure, several assumptions can be made based on the results of this thesis. In Chapter 7, the basal metabolic rate (BMR) decreased significantly when VNS was turned off, with a mean change of $2.2 \%$ up to a maximum of $7.8 \%$. After weight loss, BAT-positive morbidly obese subjects showed a mean NST of $13.7 \%$ (Chapter 4 ) compared to a mean NST of $2.4 \%$ in BAT-negative subjects. Therefore it is very likely that a large part of the $\sim 10 \%$ difference in NST between BAT-positive and BAT-negative subjects was induced by active BAT. This again corresponds with the previous suggestion that NST in BAT could increase energy expenditure in man by $\sim 5-20 \%$ of BMR. ${ }^{27}$ However, the static FDG-PET-CT imaging used in this thesis is not able to directly measure the metabolic activity of BAT, but recent dynamic imaging studies using FDG and other metabolic tracers have suggested BAT activity contributes up to $1-4.5 \%$ of BMR. ${ }^{3,30,44}$ BMR accounts for $55-65 \%$ to the total daily energy expenditure, ${ }^{27}$ which would make the contribution of BAT $\sim 2.5 \%$ ( $5 \%$ of $\mathrm{BMR}=\sim 2.5 \%$ of total daily energy expenditure) of the total daily energy expenditure (Figure 3). In summary, the results obtained in this thesis show, in combination with other reports, human BAT is related to human energy expenditure. Future studies should unravel methods to induce high levels of human BAT activity comparable to observations in rodents. In addition, possibly BAT metabolism could be positively altered in pathophysiological conditions that are accompanied with disturbed BAT activity, which would open the door for using BAT activation as a therapeutic tool in medicine.

\section{Concluding remarks and future directions}

Since Conrad Gesner in 1551 first described BAT as a tissue type he could not clearly classify as adipose tissue or muscle, the knowledge on BAT dramatically increased throughout the centuries. Especially in the last decades extensive information has emerged from rodent studies that investigated BAT into great detail. ${ }^{1}$ In this thesis BAT activity was shown to be strongly and inversely related to obesity, suggesting BAT is important in the pathophysiology of overweight. Moreover, possessing active 
BAT was affiliated with a significantly higher level of NST. This is highly interesting, since NST is a relevant component of the human energy balance and thus low NST and low BAT could be risk factors for developing overweight. Furthermore, this thesis shows that high levels of weight loss that radically change body composition are accompanied with an increase in BAT activity. This is important since it shows that low BAT activity is a consequence of obesity rather than a cause. The observed BAT recruitment upon weight loss provides hope for therapeutic options to stimulate BAT activity when low or absent. Therefore, possibly increasing or maintaining sufficient BAT activity could be beneficial for health. Future studies targeting BAT activity in obese patients could point out if stimulating BAT could be used to treat obesity and other diseases associated with low BAT activity. In addition, in this thesis human BAT was studied on a cellular level in order to give more insight in the metabolic value of human BAT. It is very likely the human 'BAT' as studied in Chapter 6 is 'beige', which provides a strong potential for a therapeutic increase in thermogenic cells in man since culturing and transplantation of beige cells was shown to be successful in rodents. If the successful transplantation of beige depots performed in mice could also be executed in man, this would provide a therapy for

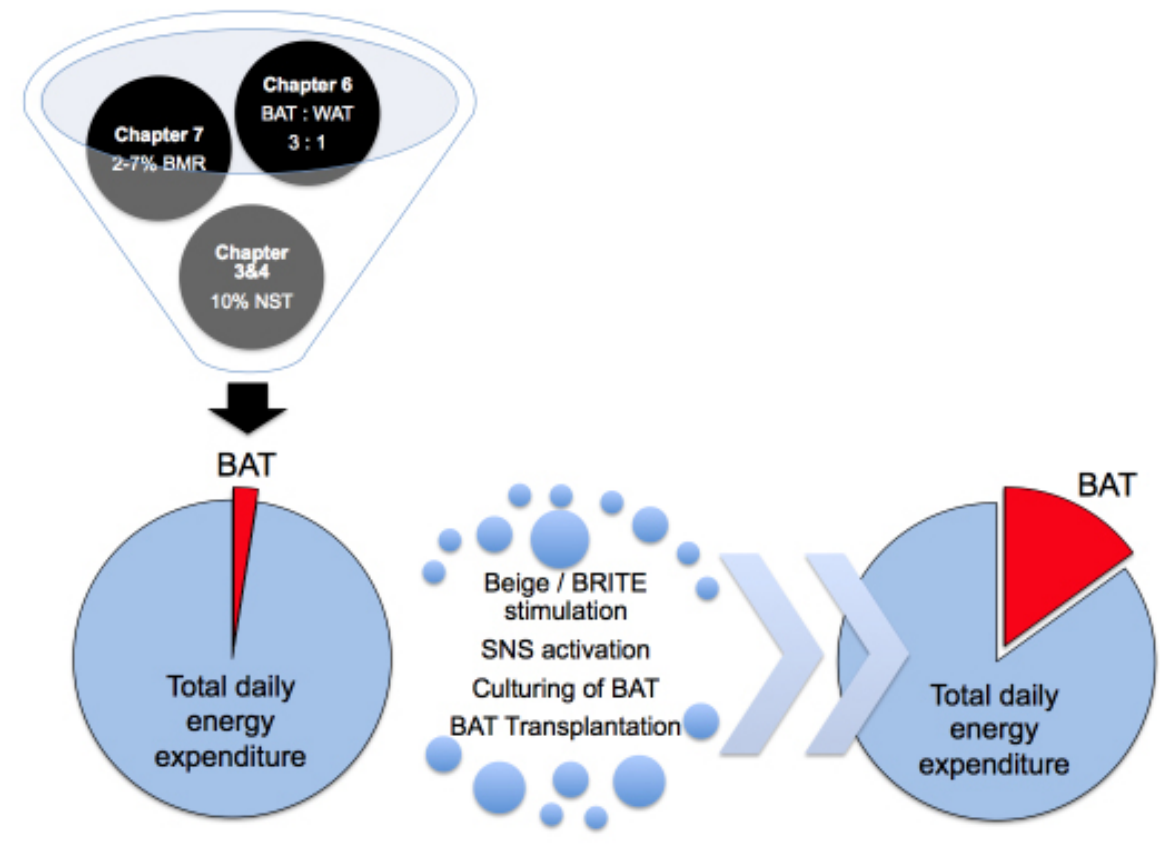

Figure 3. Estimation of the contribution of human BAT to total daily energy expenditure based on the results obtained from this thesis. Possibly, future studies targeting the factors shown in the cloud depicted in the middle could increase the contribution of human BAT. 
increasing BAT in man. Finally, this thesis investigated the possibility of stimulating BAT by electrical vagus nerve stimulation (VNS). VNS was shown to significantly increase energy expenditure, which could partly be explained by changes in BAT activity. Further research on the effect of VNS on energy expenditure and BAT activity could point out if VNS has a clinically and therapeutically significant effect on the energy balance. 


\section{References}

1. Cannon B, Nedergaard J. Brown adipose tissue: function and physiological significance. Physiol Rev 2004;84:277-359.

2. van Marken Lichtenbelt WD, Vanhommerig JW, Smulders NM, et al. Cold-activated brown adipose tissue in healthy men. N Engl J Med 2009;360:15008.

3. Virtanen $K A$, Lidell $M E$, Orava J, et al. Functional brown adipose tissue in healthy adults. N Engl J Med 2009;360:1518-25.

4. Saito $M$, Okamatsu-Ogura $Y$, Matsushita $M$, et al. High Incidence of Metabolically Active Brown Adipose Tissue in Healthy Adult Humans: Effects of Cold Exposure and Adiposity. Diabetes 2009;58:1526-31.

5. Yoneshiro T, Aita S, Matsushita $M$, et al. Brown Adipose Tissue, WholeBody Energy Expenditure, and Thermogenesis in Healthy Adult Men. Obesity (Silver Spring) 2011;19:13-6.

6. Matthias A, Ohlson KB, Fredriksswon JM, Jacobsson A, Nedergaard J, Cannon $B$. Thermogenic responses in brown fat cells are fully UCP1-dependent. UCP2 or UCP3 do not substitute for UCP1 in adrenergically or fatty scid-induced thermogenesis. J Biol Chem 2000;275:25073-81.

7. Zingaretti MC, Crosta F, Vitali A, et al. The presence of UCP1 demonstrates that metabolically active adipose tissue in the neck of adult humans truly represents brown adipose tissue. FASEB J 2009;23:3113-20.

8. Frontini A, Cinti S. Distribution and development of brown adipocytes in the murine and human adipose organ. Cell Metab 2010;11:253-6.

9. Petrovic N, Walden TB, Shabalina IG, Timmons JA, Cannon B, Nedergaard J. Chronic peroxisome proliferator-activated receptor gamma (PPARgamma) activation of epididymally derived white adipocyte cultures reveals a population of thermogenically competent, UCP1-containing adipocytes molecularly distinct from classic brown adipocytes. J Biol Chem 2009;285:7153-64.

10. Seale P, Bjork B, Yang W, et al. PRDM16 controls a brown fat/skeletal muscle switch. Nature 2008;454:961-7.

11. Seale P, Conroe HM, Estall J, et al. Prdm16 determines the thermogenic program of subcutaneous white adipose tissue in mice. J Clin Invest 2011;121:96105.

12. Tseng $\mathrm{YH}$, Kokkotou $\mathrm{E}$, Schulz TJ, et al. New role of bone morphogenetic protein 7 in brown adipogenesis and energy expenditure. Nature 2008;454:10004. 
13. Vegiopoulos A, Muller-Decker K, Strzoda D, et al. Cyclooxygenase-2 controls energy homeostasis in mice by de novo recruitment of brown adipocytes. Science 2010;328:1158-61.

14. Madsen L, Pedersen LM, Lillefosse $\mathrm{HH}$, et al. UCP1 induction during recruitment of brown adipocytes in white adipose tissue is dependent on cyclooxygenase activity. Plos One 2010;5:e11391.

15. Schulz TJ, Huang TL, Tran TT, et al. Identification of inducible brown adipocyte progenitors residing in skeletal muscle and white fat. Proc Natl Acad Sci U S A 2011;108:143-8.

16. Crisan $M$, Casteilla $L$, Lehr $L$, et al. A reservoir of brown adipocyte progenitors in human skeletal muscle. Stem Cells 2008;26:2425-33.

17. Bostrom $\mathrm{P}, \mathrm{Wu}$ J, Jedrychowski MP, et al. A PGC1-alpha-dependent myokine that drives brown-fat-like development of white fat and thermogenesis. Nature 2012;481:463-8.

18. Lee P, Swarbrick MM, Ting Zhao J, Ho KK. Inducible Brown Adipogenesis of Supraclavicular Fat in Adult Humans. Endocrinology 2011.

19. Wu ea. Beige Adipocytes Are a Distinct Type of Thermogenic Fat Cell in Mouse and Human. Cell 2012.

20. Cypess AM, Lehman S, Williams $G$, et al. Identification and importance of brown adipose tissue in adult humans. N Engl J Med 2009;360:1509-17.

21. Bouchard C, Tremblay A, Despres JP, et al. The response to long-term overfeeding in identical twins. N Engl J Med 1990;322:1477-82.

22. Yoneshiro T, Ogawa T, Okamoto N, et al. Impact of UCP1 and beta3AR gene polymorphisms on age-related changes in brown adipose tissue and adiposity in humans. Int J Obes (Lond) 2012.

23. Kingma B, Frijns A, van Marken Lichtenbelt W. The thermoneutral zone: implications for metabolic studies. Front Biosci (Elite Ed) 2012;4:1975-85.

24. Savastano DM, Gorbach AM, Eden HS, Brady SM, Reynolds JC, Yanovski JA. Adiposity and human regional body temperature. Am J Clin Nutr 2009;90:112431.

25. Christensen CR, Clark PB, Morton KA. Reversal of hypermetabolic brown adipose tissue in F-18 FDG PET imaging. Clin Nucl Med 2006;31:193-6.

26. Nedergaard J, Bengtsson T, Cannon B. Unexpected evidence for active brown adipose tissue in adult humans. Am J Physiol Endocrinol Metab 2007;293:E444-52.

27. van Marken Lichtenbelt WD, Schrauwen P. Implications of non-shivering thermogenesis for energy balance regulation in humans. Am J Physiol Regul Integr 


\section{Comp Physiol 2011.}

28. Ouellet V, Labbe SM, Blondin DP, et al. Brown adipose tissue oxidative metabolism contributes to energy expenditure during acute cold exposure in humans. J Clin Invest 2012;122:545-52.

29. Orava J, Nuutila $P$, Lidell ME, et al. Different metabolic responses of human brown adipose tissue to activation by cold and insulin. Cell Metab 2011;14:272-9.

30. Muzik O, Mangner TJ, Granneman JG. Assessment of oxidative metabolism in brown fat using PET imaging. Front Endocrinol (Lausanne) 2012;3:15.

31. van Ooijen $A M$, van Marken Lichtenbelt WD, van Steenhoven AA, Westerterp KR. Cold-induced heat production preceding shivering. $\mathrm{Br} J \mathrm{Nutr}$ 2005;93:387-91.

32. Astrup A, Bulow J, Christensen NJ, Madsen J. Ephedrine-induced thermogenesis in man: no role for interscapular brown adipose tissue. Clin Sci (Lond) 1984;66:179-86.

33. Astrup A, Bulow J, Madsen J, Christensen NJ. Contribution of BAT and skeletal muscle to thermogenesis induced by ephedrine in man. Am J Physiol 1985;248:E507-15.

34. Cypess AM, Chen YC, Sze C, et al. Cold but not sympathomimetics activates human brown adipose tissue in vivo. Proc Natl Acad Sci U S A 2012;109:10001-5.

35. Vosselman MJ, van der Lans AA, Brans B, et al. Systemic beta-Adrenergic Stimulation of Thermogenesis Is Not Accompanied by Brown Adipose Tissue Activity in Humans. Diabetes 2012.

36. Carey $A L$, Formosa MF, Van Every $B$, et al. Ephedrine activates brown adipose tissue in lean but not obese humans. Diabetologia 2012.

37. Saito M, Minokoshi $Y$, Shimazu T. Accelerated norepinephrine turnover in peripheral tissues after ventromedial hypothalamic stimulation in rats. Brain Res 1989;481:298-303.

38. Privitera MD, Welty TE, Ficker DM, Welge J. Vagus nerve stimulation for partial seizures. Cochrane Database Syst Rev 2002:CD002896.

39. Burneo JG, Faught E, Knowlton R, Morawetz R, Kuzniecky R. Weight loss associated with vagus nerve stimulation. Neurology 2002;59:463-4.

40. Bodenlos JS, Kose S, Borckardt JJ, et al. Vagus nerve stimulation acutely alters food craving in adults with depression. Appetite 2007;48:145-53.

41. Laskiewicz J, Krolczyk G, Zurowski G, Sobocki J, Matyja A, Thor PJ. Effects of vagal neuromodulation and vagotomy on control of food intake and body weight in rats. J Physiol Pharmacol 2003;54:603-10.

42. Val-Laillet D, Biraben A, Randuineau G, Malbert $\mathrm{CH}$. Chronic vagus nerve 
stimulation decreased weight gain, food consumption and sweet craving in adult obese minipigs. Appetite 2010;55:245-52.

43. Sobocki J, Fourtanier G, Estany J, Otal P. Does vagal nerve stimulation affect body composition and metabolism? Experimental study of a new potential technique in bariatric surgery. Surgery 2006;139:209-16.

44. van Marken Lichtenbelt W. Brown adipose tissue and the regulation of nonshivering thermogenesis. Curr Opin Clin Nutr Metab Care 2012. 

Summary of results 


\section{Summary of results}

The number of people suffering from obesity and its associated comorbidities is rapidly increasing and therefore it is necessary to find a therapy for this global health problem. Here, in the last decade the interest in brown adipose tissue (BAT) has increased since BAT stimulation might serve a role in the treatment of obesity. Naturally, BAT has the ability to produce heat when a subject is exposed to a cold environment. When BAT could be stimulated to produce heat, the body's energy expenditure increases and with a similar energy intake this could lead to weight loss. The activity of human BAT can be visualized by using FDG-PET-CT scans. This is an advanced scanning technique that depicts the uptake of radioactive glucose (FDG; 2-deoxy-2-18F-fluoro-D-glucose) by means of PET-CT scanning (PET-CT; positronemission-tomography-and-computed-tomography). In this thesis, FDG-PET-CT was used to measure human BAT activity. This thesis describes studies on human BAT activity in three specific groups of patients. The first group of patients was suffering from morbid obesity, an amount of overweight that causes high health risks. Therefore, these patients underwent laparoscopic adjustable gastric banding (LAGB) surgery to restrict food intake and to induce weight loss. BAT activity was measured before and one year after surgery in Chapter 3 and 4. In Chapter 6 we studied the activity of human brown and white adipocytes in a second group of patients that underwent thyroid gland surgery. We here took biopsies of adipose tissue in the areas that previously showed BAT activity on FDG-PET-CT scans. Finally, in Chapter $\mathbf{7}$ we studied patients with a vagus nerve stimulator for medically refractory epilepsy. In these patients we observed the effect of vagus nerve stimulation on energy expenditure en BAT activity.

The review in Chapter 2 showed that BAT is present and active in humans and possibly serves an important physiological role. Moreover, from a clinical point of view the relation between BAT activity and energy expenditure suggests the stimulation of BAT activity might be a new therapeutic target to treat obesity. Furthermore, evidence from in vivo and in vitro studies showed that altered BAT activity could also be relevant in the pathophysiology of the metabolic syndrome (type 2 diabetes, dyslipidemia, hepatosteatosis), thyroid disease, pheochromocytoma, cancer cachexia, hibernoma and the immune system. Based on the literature available, therapeutic suggestions are made to stimulate BAT by cold, insulin, the central nervous system, bile acids and gut hormones. A summary is provided of molecular studies that show possibilities for the development of de novo BAT derived from other types of adipocytes or their progenitors. Finally, 
targets for future studies on the role of BAT in disease are discussed. In Chapter $\mathbf{3}$ we studied BAT activity in morbidly obese subjects and showed that - in combination with our previous findings in lean subjects - BAT activity was strongly and inversely related to body mass index (BMI) and body fat percentage (BF\%). The morbidly obese subjects showed no BAT activity (BAT-negative) or lower BAT activity compared to lean subjects (BAT-positive). There was a significantly lower increase in energy expenditure upon cold exposure (non-shivering thermogenesis (NST)) in BAT-negative compared to BAT-positive subjects. This is important, since it may indicate that BAT is involved in human energy expenditure upon cold exposure. To unravel the observed low BAT activity in obesity in Chapter 3, we studied a group of morbidly obese patients selected for bariatric surgery in Chapter 4 . In this group, we investigated the effect of weight loss induced by bariatric surgery and found an increased BAT activity after weight loss. Strikingly, after weight loss BAT became active in regions that were BAT-negative before weight loss, showing for the first time recruitment of BAT in man. Again, after weight loss BAT-positive subjects had a significantly higher level of NST compared to BAT-negative subjects. These findings BAT could be important in weight management in obesity. Most dedicated studies on BAT activity show BAT becomes active after cold exposure and suggest an important contribution of BAT to non-shivering thermogenesis (NST). ${ }^{6}$ Interestingly, in man NST has also been associated with skeletal muscle mitochondrial uncoupling. ${ }^{7}$ Therefore, we investigated the effect of weight loss induced by bariatric surgery on skeletal muscle metabolism in Chapter 5. Our results show the level of skeletal muscle mitochondrial uncoupling remains unaffected by weight loss induced by bariatric surgery. This is in line with previous reports that did not show changes in skeletal muscle metabolism after diet-induced weight loss. However, in Chapter 5 we showed skeletal muscle mitochondrial ADP-dependent state 3 respiration significantly increased after high levels of weight loss as observed after bariatric surgery. This suggests that, in addition to a beneficial effect on BAT activity and NST, bariatric surgery positively affects skeletal muscle metabolism but not thermogenesis.

Both studies performed in Chapter $\mathbf{3}$ and $\mathbf{4}$ used FDG-PET-CT imaging to non-invasively quantify BAT activity. In Chapter 6, we determined cellular oxygen consumption in human adipose tissue obtained from the neck region that is known to be FDG-PET-CT-positive for BAT. Strikingly, in these tissue biopsies we observed an on average three times higher cellular mitochondrial respiration compared to subcutaneous white adipose tissue (WAT) from the same subject, measured under unstimulated conditions. These results show that adipose tissue from the 
human BAT region has an increased metabolism even without external stimuli. To therapeutically induce BAT activity, in Chapter 7 we explored the effect of vagus nerve stimulation on BAT activity in refractory epilepsy patients. Our results show that during active VNS there is a significantly increased energy expenditure. The observed increase in energy expenditure upon VNS was related to the change in BAT activity, suggesting the effect of VNS on energy expenditure is partly due to changes in BAT activity.

In the last years the knowledge on human BAT has strongly expanded. In this thesis, human BAT activity was studied and we observed a relation between BAT activity and obesity. This suggests low BAT activity could be important in the development of obesity. Fortunately, BAT activity increases after weight loss and therefore BAT could possibly be meaningful in weight management and health. Future studies aiming for increasing human BAT activity could possibly provide new options for the treatment of obesity. 

Dutch summary 


\section{Samenvatting}

Bruin vet is bekend sinds 1551 toen de Zwitserse bioloog Conrad Gessner bij het ontleden van marmotten een weefsel vond dat hij "noch als vet, noch als vlees" beschreef. In de eeuwen daarna werd er steeds meer bekend over bruin vet en in 1972 volgde de eerste gedetailleerde anatomische omschrijving van menselijk bruin vet. Bruin vet zit bij volwassenen rond de grote vaten en spieren in de hals, boven het sleutelbeen, langs de wervelkolom en rond de nieren.

Bruin vet verschilt duidelijk van wit vet; de witte vetcel heeft de capaciteit om overtollige energie vast te houden en bestaat met name uit een grote vacuole (blaas) waarin vetzuren worden opgeslagen voor gebruik in tijden van energietekort. Wanneer er sprake is van een overmatige hoeveelheid wit vet spreken we van overgewicht of obesitas. De bruine vetcel heeft een cytoplasma (celvloeistof) met daarin een grote hoeveelheid mitochondriën (energiecentrale) en bevat meerdere kleine vacuoles (blaasjes) gevuld met vetdruppels. De mitochondriën in bruine vetcellen zijn donker gekleurd en geven bruin vet zijn karakteristieke kleur, die in de praktijk varieert van karamelkleurig tot bruin/donkerrood. Naast een kleurverschil zijn er een aantal belangrijke eigenschappen die bruin vet onderscheiden van wit vet. De functie van bruin vet is het belangrijkste verschil met wit vet. Het is inmiddels aanvaard dat bruin vet energie gebruikt om warmte te produceren, in tegenstelling tot wit vet dat vooral functioneert als opslag van energie. Bruin vet kan die warmte produceren door een uniek proces dat plaats vindt in het mitochondrium van de bruine vetcel. Dit proces heet mitochondriële ontkoppeling, mogelijk gemaakt door het ontkoppelingseiwit 1 (uncoupling protein 1 (UCP1)). Tijdens de mitochondriële ontkoppeling in de bruine vetcel wordt warmte geproduceerd uit energie die in andere cellen gebruikt zou worden als bouwstof voor het lichaam. In de bruine vetcel wordt deze energie niet als bouwstof benut, maar gebruikt om het lichaam te verwarmen in een koude omgeving. Ten slotte is bruin vet ten opzichte van wit vet zeer goed doorbloed en heeft een rijke sympatische zenuwvoorziening.

Omdat overgewicht een steeds grotere rol speelt in onze samenleving is de interesse in het functioneren van bruin vet de laatste jaren toegenomen. Hierbij wordt met name gedacht aan een rol voor bruin vet stimulatie als behandeling van overgewicht. Als bruin vet wordt gestimuleerd tot warmteproductie wordt er meer energie gebruikt door het lichaam; bij een onveranderd eetpatroon zou dat kunnen zorgen voor gewichtsverlies.

De activiteit van bruin vet kan bij de mens zichtbaar worden gemaakt door bij milde koude een FDG-PET-CT scan te maken. FDG-PET-CT is een geavanceerde 
scantechniek, waarbij radioactieve glucose (suiker) wordt ingespoten. De stofnaam voor deze radioactieve glucose is FDG (2-deoxy-2-18F-fluoro-D-glucose), waarbij PET-CT (positron-emission-tomography-and-computed-tomography) de scantechniek is om de locatie in het lichaam van het opgenomen FDG af te beelden. Deze techniek hebben we gebruikt om de activiteit van bruin vet te meten. Dit proefschrift bevat studies naar de activiteit van bruin vet in gezonde proefpersonen en patiënten met een bepaald ziektebeeld. In dit proefschrift worden de resultaten beschreven van onderzoeken gedaan bij drie groepen patiënten. De eerste groep patiënten had ernstig overgewicht (morbide obesitas), waarbij er zoveel overgewicht was dat er sprake was van een groot gezondheidsgevaar. Daarom werd bij deze patiënten operatief een maagband geplaatst om te zorgen voor een beperking in voedselinname om af te vallen. De activiteit van bruin vet bij deze patiënten wordt voor de operatie en een jaar na de operatie beschreven in hoofdstuk 3 en 4. In hoofdstuk 6 onderzochten we bij een tweede groep patiënten de activiteit van bruine en witte vetcellen, afgenomen op plekken in de hals waar in eerdere onderzoeken op de FDG-PET-CT scan bruin vet activiteit werd gezien. We hadden bij deze patiënten de mogelijkheid om biopten (stukjes weefsel) van bruin en wit vet te nemen omdat ze een schildklieroperatie ondergingen. Daarbij is het nodig spieren en vet in de hals bloot te leggen om de schildklier goed te kunnen zien; daardoor hadden we de mogelijkheid om bruin en wit vet biopten te nemen in het operatiegebied. Als laatste wordt in hoofdstuk 7 de derde onderzoeksgroep beschreven met patiënten die een elektrische nervus vagus zenuwstimulator hebben om epilepsie te behandelen. Wij onderzochten bij deze patiënten of zenuwstimulatie bruin vet kan activeren. Bruin vet activiteit werd met FDG-PET-CT scans gemeten nadat de stimulator aan en uit stond.

In dit proefschrift wordt in hoofdstuk 2 een literatuur overzicht gegeven van eerdere klinisch georiënteerde onderzoeken naar bruin vet in de mens. De belangrijkste uitkomsten zijn: bruin vet activiteit laat een positief verband zien met de toename van energieverbruik in een koude omgeving en bruin vet activiteit is negatief gerelateerd aan van overgewicht. Daarnaast zijn er aanwijzingen dat bruin vet activiteit een rol speelt bij ziektes als type 2 diabetes, vetstofwisselingsstoornissen, vervette lever, schildklierziekten, kanker en afwijkingen in het immuunsysteem. Bruin vet zou mogelijk gestimuleerd worden door langere tijd in een koude omgeving te verblijven, door de zenuwen die bruin vet activeren elektrisch of met medicatie te stimuleren of door hormonen toe te dienen die de activiteit van bruin vet in cel- en dierstudies verhoogden. Hoofdstuk 3 beschrijft ons onderzoek naar bruin vet activiteit bij obesitas. De resultaten laten zien dat bruin vet activiteit zeer 
laag of zelfs afwezig is bij morbide obesitas en bij de patiënten zonder bruin vet activiteit was de warmteproductie in een koude omgeving veel lager. Nadat deze patiënten een maagbandoperatie hadden ondergaan en gemiddeld 36 kilogram slanker werden onderzochten wij opnieuw de bruin vet activiteit in hoofdstuk 4. Een jaar na de operatie werd er bij vier van de tien patiënten een toename van bruin vet activiteit gezien. Dit suggereert dat ernstig overgewicht de koude activatie van bruin vet voorkomt. Mogelijk kan dit van invloed zijn op het handhaven van een gezond gewicht. Naast bruin vet kan ook spierweefsel zijn mitochondriën voor ontkoppeling gebruiken. Hierdoor wordt in een koude omgeving in de spieren warmte geproduceerd zonder te rillen. In hoofdstuk $\mathbf{5}$ onderzochten wij in dezelfde morbide obese patiënten de functie van mitochondriën in spiervezels voor en na gewichtsverlies door een maagbandoperatie. De resultaten laten zien dat de activiteit van spiermitochondriën toenam na gewichtsverlies. Dit suggereert dat gewichtsverlies door middel van een maagbandoperatie naast een toename in bruin vet activiteit ook de spierstofwisseling kan laten toenemen. In hoofdstuk 6 werd het zuurstofverbruik van bruine vetcellen vergeleken met witte vetcellen van dezelfde persoon. Deze cellen waren afkomstig uit het halsgebied van patiënten die een schildklieroperatie ondergingen. Er werd weefsel afgenomen uit het gebied dat in de onderzoeken in hoofdstuk 3 en 4 op de FDG-PET-CT scan bruin vet activiteit liet zien. Omdat er nog eerder onderzoek is gedaan naar de activiteit van menselijke bruin vetcellen moest er een protocol worden ontwikkeld, waarbij gebruik gemaakt werd van de kennis opgedaan uit onderzoek naar menselijk wit vet en bruin vet van dieren. De resultaten die werden verkregen met gebruik van het ontwikkelde protocol laten zien dat het zuurstofverbruik in vetweefsel dat werd afgenomen op de plek die eerder op FDG-PET-CT scans bruin vet activiteit liet zien drie keer hoger is dan in witte vetcellen van dezelfde patiënt. Dit suggereert dat de menselijke bruine vetcel buiten het lichaam, zonder de toevoeging van stoffen die bruin vet activeren, een hoge stofwisseling heeft. Hoofdstuk 7 beschrijft het effect van zenuwstimulatie (nervus vagus stimulatie) op het energieverbruik en bruin vet activiteit. Er werd gezien dat zenuwstimulatie het energieverbruik verhoogt. Ook was er een relatie tussen de stijging van het energiegebruik en bruin vet activiteit. De stijging in energieverbruik kan dus voor een deel veroorzaakt zijn door bruin vet activatie.

Sinds Conrad Gessner in 1551 bruin vet voor het eerst beschreef is de kennis over bruin vet sterk toegenomen. Met name in de afgelopen jaren is er veel informatie verkregen uit studies in cellen en muismodellen. In dit proefschrift werd bruin vet activiteit onderzocht in de mens en werd er een relatie gezien tussen bruin vet 
activiteit en overgewicht. Dit suggereert dat een lage bruin vet activiteit belangrijk kan zijn bij het ontstaan en in stand blijven van overgewicht. Mogelijk is een lage bruin vet activiteit een risicofactor voor het ontwikkelen van overgewicht. Gelukkig is er een toename in bruin vet activiteit na fors gewichtsverlies. Behandelingen gericht op het stimuleren van bruin vet kunnen mogelijk een positieve invloed hebben op het gewicht en de gezondheid. Toekomstige studies gericht op het verhogen van bruin vet activiteit bij mensen met overgewicht kunnen mogelijk nieuwe opties geven voor het behandelen van obesitas. 

Dankwoord 


\section{Dankwoord}

Ik kan het bijna niet bevatten, maar het is zover; het proefschrift is af en de datum staat vast! In 2009 begon ik als verse drs. aan de uitdaging die er uiteindelijk toe zal leiden dat die -s er binnenkort af gesnoept kan worden! Het was echter nooit zover gekomen als ik niet zo ontzettend veel hulp, steun en motivatie had ontvangen van alle mensen om mij heen. Bij dezen wil ik daarom iedereen waarmee ik heb mogen samenwerken ontzettend bedanken voor de mooie tijd en gezelligheid die het mogelijk maakten deze enorme berg werk te verzetten.

Allereerst wil ik alle patiënten bedanken die tijd vrijmaakten om mee te doen aan onze studies. Bedankt voor alle bezoeken, uren in de kou, spierpijn en donatie van biopten ten behoeve van de wetenschap. Een aantal van $u$ heb ik mogen volgen in uw periode van gewichtsverlies na de operatie. Hier was het indrukwekkend om te zien hoe sociale levens weer opbloeien en mensen opeens weer midden in het leven staan doordat het overgewicht afneemt. Hoewel bruin vet daar soms ver weg van lijkt te staan, ligt hier voor mij wel de basis van mijn motivatie om iedere dag mijn werk te doen. Of het nu overgewicht betreft of zoveel andere ziektebeelden die ik tegenwoordig voorbij zie komen; uiteindelijk gaat het erom mensen te helpen.

Een speciaal woord van dank wil ik aan iedereen geven die mijn promotie mogelijk heeft gemaakt, met in het bijzonder de volgende mensen:

Mijn co-promotores Nicole, Wouter en mijn promotor Patrick. Nicole, toen ik in mijn derde studiejaar op jouw kamer kwam om te vragen hoe je nu het best chirurg kunt worden heb je mij direct onder je vleugels genomen. Direct kon ik beginnen met wetenschappelijk onderzoek, waarbij jouw altijd aanwezige enthousiasme zo aanstekelijk heeft gewerkt dat het uiteindelijk tot dit proefschrift heeft geleid. Ik wil je voor zoveel zaken bedanken; het 'regelen' van een baan direct na mijn studie, het 'lobbyen' bij alle disciplines om onze studies mogelijk te maken, de begeleiding tijdens mijn klinische en wetenschappelijk werk en je interesse in mijn persoonlijke leven. Ik kon altijd bij je terecht, waarvoor ik je zeer dankbaar ben. De manier waarop jij je werk doet en altijd opgewekt bent dwingt bij mij groot respect af en dienen als voorbeeld. Wouter, toen ik als 'clinicus' met het bruin vet onderzoek bij jou begon was mijn kijk op een promotietraject een stuk anders dan nu. Ik wilde zo snel mogelijk zo veel mogelijk doen en had daarbij absoluut geen tijd om te wachten op wat dan ook. Gelukkig wist jij me ervan te overtuigen dat er meer is 
dan het 'doel', namelijk de weg waarop je je bevindt. Hierbij denk ik met een warm gevoel terug aan een avond in een Londense pub waar we dit onderwerp onder het genot van vele pints en ales hebben besproken. De discussie vlamde op naar een niveau waarop Boris het uiteindelijk met een echte oorvijg van jou moest bekopen, maar die avond is mij wel duidelijk geworden waar jij het over had; althans, dat denk ik nu tenminste.. Ik wil je bedanken voor je geduld en een vorm van dagelijkse begeleiding waarbij je altijd tijd hebt voor je promovendi. Ook naast het werk op de universiteit waren we altijd welkom om mee te varen op de Iguana en in jullie prachtige huis wijntjes te komen drinken. Onder mijn optimisme is de boot de eerste keer onder de Kennedybrug door gevaren, een mooi moment dat ik niet vergeet. Patrick, ik heb diep respect voor jouw gedrevenheid als wetenschapper. Toen ik je leerde kennen kon ik niet geloven dat iemand zo snel zo veel kan bereiken. Die snelheid heb ik mogen ervaren wanneer ik een manuscript na maanden werk opstuurde en enkele uren later, meestal in de avond, terug kreeg met gedetailleerd en gevat commentaar. Door jouw hulp is mijn promotie vlot verlopen en ik hoop dat je me inmiddels vergeeft dat ik tijdens ons wielrentochtje een keer net iets sneller boven was dan jij. Ik wil je bedanken voor je begeleiding als promotor.

I would like to thank professor Van Baak, professor Daanen, professor Hesselink, professor Mottaghy and dr. Virtanen for critically evaluating this thesis. Dr. Virtanen, thank you for travelling from Finland to be part of the corona.

De basis van promotieonderzoek werd gelegd tijdens mijn WESP-stage in een verlaten cardiolab op de derde verdieping. Maartje, Ruben, Kirsten en Dennis, bedankt dat jullie me toe hebben gelaten in jullie domein en ik hoop in de toekomst nog eens met jullie te mogen samenwerken.

$\mathrm{Na}$ mijn studie werd ik door Nicole Bouvy en Sven van Helden aangenomen als arts-assistent bariatrische chirurgie. Sven, bedankt voor de mogelijkheid te assisteren op de OK en klinische ervaring op te doen. Ik hoor veel goede verhalen uit Zwolle en hoop je op 7 juni weer te mogen spreken om bij te praten. In deze tijd deelde ik een kamer met Stella, Magda en Bart. Stella en Magda, als echte dames ondersteunden jullie Bart en mij tijdens als ons gemopper. Bedankt voor de leuke gesprekken, adviezen en het geweldig lekkere Italiaanse avondje in Ceramique. Bart, Bartje, BPPvW, we hebben veel plezier gehad op deze 'inpandige' kamer zonder direct daglicht en op de poli waar jij net als ik vaak met enigszins aparte patiënten te maken had. Ik heb respect voor je gedrevenheid en was altijd zeer 
onder de indruk als je weer een A4-tje op de muur plakte met je zoveelste artikel in korte tijd. Ik wil je bedanken voor je kameraadschap en hoop ook binnenkort op jouw feestje te mogen komen. Tijdens mijn werk op de polikliniek chirurgie werd ik altijd gesteund door Bernadette en consorten, bedankt voor jullie hulp en flexibiliteit. Ook wil ik Evelien Goldberg in het bijzonder bedanken, als secretaresse van Nicole was jij altijd bereid te helpen, veel dank daarvoor. In deze periode kon ik door de hulp van Johanne Bloemen op maandagavond plaats nemen als adviseur in de commissie onderzoeksbeleid (COB). Daar zag ik alle subsidieaanvragen en hoogleraar-aanstellingen voorbijkomen, een zeer leerzame en leuke functie. Ilske, Marco, Margreet en professor Ten Cate, bedankt voor de leuke tijd in de COB. Toen ik na een tijdje had gezien hoe zo'n aanvraag in zijn werk ging besloot ik er zelf een te schrijven. $\mathrm{Na}$ een pittige presentatieronde kwam Ilske uiteindelijk met het goede nieuws dat ik een van de drie (uit dertig!) gehonoreerde aanvragen kreeg. Nog steeds ben ik enorm blij met deze Kootstra Talent Fellowship die het voor mij mogelijk maakte fulltime wetenschappelijk onderzoek te gaan doen. Johanne, zonder jouw tip in de COB plaats te nemen was dit misschien nooit mogelijk geweest, bedankt daarvoor.

Vanaf november 2010 werd ik voortaan gestationeerd op kamer 2.306 bij de afdeling Humane Biologie. Op deze afdeling heb ik als klinisch georiënteerd arts geleerd hoe basaal wetenschappelijk onderzoek in zijn werk gaat en gezien met hoeveel precisie en geduld dit gepaard gaat. Zonder de hulp van bijna iedereen op deze afdeling was dit proefschrift nooit tot stand gekomen. In het bijzonder wil ik de mensen bedanken waar ik direct mee samen heb gewerkt. Mijn kamergenootjes Madeleen, Bianca en Hasibe, bedankt voor jullie verdraagzaamheid. Als enige mannelijke kamergenoot heb ik een mooie en gezellige tijd gehad met jullie. Madeleen en Bianca, toen ik dacht dat mijn arbeidsethos wel in orde was leerde ik jullie kennen en kwam erachter dat ik helemaal niet zo hard werk. Jullie waren vaak zeven dagen per week dag en nacht met jullie onderzoek bezig, iets waar ik met verbazing naar keek. Gelukkig kwam ik jullie regelmatig tegen tijdens de spinsessies in // Fiore, een teken dat jullie ook sportief op topniveau zaten. Ik heb veel respect voor jullie gedrevenheid waardoor jullie zeker gerenommeerde wetenschappers gaan worden. Madeleen, ik wens je veel plezier en succes in Stockholm, ontzettend gaaf dat je in deze hippe stad je carrière kunt vervolgen. Bianca, soms ook wel het muizenmeisje genoemd, je bent een doorzetter als geen ander, veel plezier en succes in de toekomst! Vanuit mijn basis op kamer 2.306 begon de werkdag meestal met een bakkie met de andere collega's een paar kamers verderop. In de eerste kamer zaten Anouk, Mark, 
Christel en later Lisje. Anouk, nadat je terug kwam uit Afrika ben je ook in de bruin vet hype terecht gekomen. De acclimatisatiestudie is tot nu toe de kroon op je werk en laat zien dat je enorm gedreven bent. Ik wil je bedanken als betrokken collega en voor je vermogen om mij weer met beide benen op de grond te zetten als ik weer eens iets te hooghartig werd. Mark, Hanssen, Handsome, jij was bij binnenkomst al een topper en weet zelf heel goed waar je mee bezig bent. Je bent een mooie vent (letterlijk en figuurlijk ;), bedankt voor de leuke tijd. Christel, Crystal, de koningin van de respiratiekamers, bedankt voor je Brabantse gezelligheid. Lisje, ook jij bent vanuit Eindhoven naar het Maastrichtse gekomen, ik wens je veel succes en wil je bedanken voor de leuke uitjes en mooie avondjes. In de volgende kamer heerste er een aparte sfeer die ik misschien wel nooit meer mee mag maken. Boris, Erik en Maarten, wat zijn jullie samen topgasten! Het was altijd een gekkenhuis bij jullie; balletje-balletje, hardcore-kwartiertje, Dumpert, altijd wat te doen en voor mij vaak een opkikker om uit de dagelijkse sleur te komen. Erik, Knallert, we hebben elkaar goed leren kennen en ik heb diep respect voor jouw authenticiteit. Je blijft altijd jezelf en wist je door een moeilijke periode te slepen. Een hoogtepunt was jullie bruiloft, ik hoop je nog vaak te zien en nog veel feestjes met je te mogen maken. Boris, B-to-the-K, zonder jouw hulp had ik dit proefschrift écht niet kunnen schrijven. Je hielp met veel geduld bij mijn berekeningen en was nooit te beroerd om mee te denken. Naast het werk zijn we goede vrienden geworden met mooie Julius Caeser en Stalin pokeravonden, casinoritjes, een ludieke zwemtocht naar het schateiland en een prachtige promotie. Ik waardeer je keus om je gevoel te volgen en de wetenschappelijke wereld even te ontspringen. Maarten, Vosje, sinds je aanstelling in november 2009 hebben we veel lol gehad. Op congressen waren we vaste kamergenoten, waarbij we in Stockholm en Istanbul vergezeld werden door onze goede vriend dr. Death; 'don't worry, we are doctors'. We hebben veel en intensief samen gewerkt, waarbij jij altijd rust en overzicht houdt en de juiste balans tussen werk en privé kent. Op maandag altijd even kijken of je de voorpagina van de Limburger weer gehaald hebt en een carrière als filmster in Allstars zijn maar een paar voorbeelden van de mooie tijd als collega's. Samen waren we Boris' paranimf en ik ben enorm blij dat je ook bij mij aan mijn zijde wilt staan, het belooft een 
mooie dag te worden. Evie, jij bent mijn bench-to-bedside opvolger en werd direct op het zware thyroidproject geplaatst. Je bent een nuchtere, hardwerkende arts, ik wens je enorm veel plezier en succes met je promotietraject.

Op niveau 0 werd echt gewerkt en wil ik een aantal collega's bijzonder bedanken. Esther Phielix, als oxygraafleerling wist je mij wegwijs te maken in het lab en later hebben we samen op internationaal niveau de - lange termijn - thyroidstudie op weten te zetten. Bedankt voor de introductie in de wetenschap. Tineke, onze samenwerking was altijd erg gemoedelijk en je ging zelf als een speer door je promotietraject. Je keus voor een huis over de grens en een zwangerschap kenmerken je en ik weet zeker dat op korte termijn hoogleraar radiologie bent. Lena en Silvie, jullie waren altijd in voor een praatje en een drankje, bedankt voor de gezelligheid. Lauren, thank you for guiding me through the extremely complicated process of developing our method te measure human BAT and WAT. I really enjoyed our time and wish you all the best in the States. Gert en Esther, bedankt voor jullie bereidwilligheid al dat vet- en spierweefsel te snijden, kleuren en op beeld vast te leggen. Joris en Johanna, jullie zijn een uniek Nederlands/ Zweeds paar en als collega's hebben we prettig samen kunnen werken. Johanna, bedankt voor de eerste muizenmetingen en Joris bedankt voor al je hulp bij het analyseren en schrijven. Guy P., mijn naamgenoot en Bram, de Belgische charmeur, we hebben veel lol gehad tijdens de vrijmibo's en daaropvolgende kroegavonden, waarvoor dank. Jos, zonder jou waren die borrels er nooit geweest. Ondanks het soms matige enthousiasme van anderen voor een biertje regelde jij altijd een goed gevulde koude kamer. Het was erg gezellig. Gijs Goossens, de tourpoolwinnaar en koffiedrabkijker, het was mooi.

Dames van het secretariaat, Desiree, Claudia en Yolanda, bedankt voor alle paaseitjes, scans en koffiepraat.

Loek, Paul en Laurens, bedankt voor de onvoorwaardelijke ondersteuning van alle gammele Omnicals en Oxycons.

Alle FDG-PET-CT scans om de vaak minimale hoeveelheden bruin vet in kaart te brengen waren onmogelijk geweest zonder de gastvrijheid en hulp die ik kreeg op de afdeling Nucleaire Geneeskunde. Dames van het secretariaat, professor Teule, 
Boudewijn Brans, Jan Bucerius, dr. Van Kroonenburgh, dr. Prompers, Stefan, Cecile, Emiel, Cristel, Kyra, Servais, Mariëlle, Christian, Roel, Denise, Gerrit, bedankt voor de ondersteuning, leuke gesprekken en begrip voor onze bruin vet fobie.

Naast mijn collega's bij HB had ik regelmatig contact met de onderzoekers van de Heelkunde op de vijfde verdieping. Charlotte en Froukje, bariatric twins, we hebben mooie IFSO-congressen mogen meemaken en een leuke samenwerking gehad. Froukje, het is fijn te zien dat je bij de KNO thuis bent. Charlotte, voor jou de laatste loodjes en dan een mooi feestje, in de toekomst misschien wel weer collega's? Jan-Willem Greve, bedankt voor het beoordelen van de stukken die ik samen met Ruben heb geschreven en de heerlijke fruits de mer in Parijs. Ruben Schouten, je promotie naast je opleiding, voor jou een peulenschil. Bedankt voor het samen schrijven van twee artikelen en je optimisme. Verder wil nog alle andere collega's bedanken: Simon, Kostan, Dirk, Joep, 'Mo', Sander, Kaatje, Bas, Aron, Edgar, Freek, Liliane, Mechteld, Maarten, Marlou, Nina, Kevin, Kim, Tiara, Givan, Tim, Eva. Pieter, na onze ervaring in Heerlen zijn we samen gaan promoveren. We zagen elkaar vooral veel op de fiets waar we zo hard mogelijk door het Limburgs landschap probeerden te scheuren, bedankt voor de mooie tochtjes.

Bij ons thyroidproject hebben we veel hulp gekregen van de afdelingen Endocrinologie en Klinische chemie. Professor Schaper en Bas Havekes, bedankt voor de prettige samenwerking en succesvolle start van dit project. Judith Bons, dr. Menheere en Jan Bleus, bedankt voor de hulp om alle schildklierhormoonaanvragen in kaart te brengen.

Nadat ik in juli 2012 uit Maastricht met de stroming van de Maas mee in Rotterdam terechtkwam, ben ik met open armen in het Sint Franciscus Gasthuis ontvangen. Het was voor mij als Limburger niet niks om de Randstad op te zoeken, maar ik voelde me er snel thuis door het enorme enthousiasme en plezier van mijn collega's. Het spreekwoordelijke warme bad waarover in het SFG vaak wordt gesproken bestaat echt, ik kon er namelijk direct in gaan zitten. Bas, An, Joris, Maykel, Dennis, Talitha, Diederick, Brechtje, Kristel, Nieke, Nienke, Roel, Eva, Marin, Ralph (collega), MC, Sandra, Avi, Guyon, Arezo, Bianca, Ralf en Stefanie, bedankt voor een geweldige 
werksfeer. Alle leden van de maatschap wil ik bedanken om als ANIOS klinische ervaring op te mogen doen in het SFG. Dr. Mannaerts, Guido, bedankt voor de steun tijdens mijn sollicitatieproces, nu duimen maar.

Aan mijn middelbare schooltijd in Geleen heb ik een aantal mooie vriendschappen mogen overhouden die ik door de jaren heen steeds meer ben gaan waarderen. Sjoerd, Jef, Jordy, Mark, we zien elkaar nog vaak en hebben altijd prachtige avonden. Bij jullie is er altijd lol, zelfs als Haazen even de weg kwijt is in Chicago city. Dat we nog maar veel mooie avonden in café het Molentje aan de Debeijestraat mogen hebben. Samef, Sander, Cas, Faro, Wim, Pie, Moniek, Max (creatief brein achter dit boekje!), bedankt voor alle leuke huisfeestjes, Boxing Days en carnavalszondagen. Stef, Bart en Daniël, ook al komen jullie niet direct uit Geleen en omstreken, jullie zijn inmiddels wel stevig geïntegreerd in de vriendengroep, dank voor de mooie momenten. Samef en San, als Maastrichtse crew hebben we veel pintjes mogen weghakken, ik vond het altijd enorm gezellig.

De geneesco's, in ons derde studiejaar ontstond op het Herdenkingsplein een vriendengroep die in de jaren daarna voor veel leuke herinneringen heeft gezorgd. Jos, Ramon, Caro, Raph, Bart, Marieke, Mirte, Laura, Elsemieke, Marie-Claire, bedankt voor jullie vriendschap. Peeters, sinds dag 1 zijn we dikke matties, laten we die Ronde van Vlaanderen gewoon doen! Marcus, De Bemel, het moment dat we jou verloren was bizar, we missen je enorm.

Marc Schreinemacher, je hebt me geïntroduceerd in de wetenschap en we mochten zelfs samen publiceren. Het was altijd dikke pret, hopelijk zien we elkaar door de kortere afstand wat vaker in de toekomst.

Plons, gasten, wat hebben wij al veel mooie momenten mogen meemaken. We zijn net terug uit India waar we ons 10-jarig lustrum op gepaste wijze hebben mogen vieren. Rick, Krijn (Krinos), Gabo (Gabie), Raph (De Boy), Bennie (Benz), Antoine (Tonnetje), Niels (Nilus), op naar de volgende 10! Gabs, Raph en Lotte, bedankt voor al die limoncello-woensdagavondjes. 
Ramon, kerel, je werd aanvankelijk in de familie geïntroduceerd als nieuwe aanwinst. Al snel werden we los hiervan goede vrienden en hebben we een klik die ik met niemand anders heb, daarvoor wil ik je bedanken. We studeerden samen, sporten samen en delen lief en leed. We think alike, ik ben ontzettend blij dat je mijn paranimf wilt zijn.

Mijn familie heeft me altijd gesteund en begrip getoond. Pap en mam, bedankt voor al jullie aandacht, steun en vertrouwen, jullie zijn geweldige ouders. Ralf en Pascal, als broers zijn we de beste vrienden, ik ben trots dat ik jullie broer mag zijn. Maudje, je bent eigenlijk gewoon een zesde familielid, bedankt dat je je hebt laten inlijven bij de familie Vijgen. Oma en opa, bedankt voor al jullie aandacht en enthousiasme voor jullie oudste kleinzoon. Opa, je was mijn beste vriend, ik mis je enorm. Lisette, Leo, Vera, Floor, Ward, Julie, Tijn, Timme, bedankt voor jullie interesse en gezelligheid. Wim, Marie-Josée, Miriam, Paul, Ellen, Paul, Annette, Marie, Lena, Paul-Lucca, Sjir, bedankt voor alle leuke momenten. Ook de familie van Kats wil ik bedanken voor de hartelijke ontvangst, leuke feestjes en verdraagzaamheid voor die Limburgse jongen. Kees, Margriet en Koen, heel erg bedankt.

Lieve Maart, in ons tweede huwelijk gaat het beter dan ooit. Ik ben helemaal gek van je en zal dat altijd blijven. 

List of publications 


\section{Published papers}

Vijgen $\mathrm{GH}$, van Marken Lichtenbelt WD. The clinical impact of brown adipose tissue: therapeutic potential of a re-discovered thermogenic organ. Accepted in Frontiers in Bioscience.

Vijgen GH, Schouten R, Bouvy ND, Greve JW. Salvage banding for failed Roux-en-Y gastric bypass. Surgery for Obesity and Related Diseases. 2012 Nov-Dec;8:803-8.

Vijgen GH, Bouvy ND, Smidt M, Kooreman L, Schaart G, van Marken Lichtenbelt W. Hibernoma with metabolic impact? BMJ Case Rep. 2012 Aug 21;1-3.

Beijer E, Schoenmakers J, Vijgen GH, Kessels F, Dingemans AM, Schrauwen P, Wouters $M$, van Marken Lichtenbelt WD, Teule GJJ, Brans B. A role of active brown adipose tissue in cancer cachexia? Oncology Reviews. 2012 Jan;6:88-94.

Vijgen GH, Bouvy ND, Teule GJJ, Brans B, Hoeks J, Schrauwen P, van Marken Lichtenbelt WD. Increase in brown adipose tissue activity after weight loss in morbidly obese subjects. JCEM. 2012 Jul;97:1229-33.

Vijgen GH, Pelzers L, Schouten R, Greve JW, van Helden SH, Bouvy ND. Rebanding of laparoscopic adjustable gastric banding: success or failure? Obes Surg. 2012 Feb;22:287-92.

Vijgen GH, Bouvy ND, Schrauwen P, Teule GJJ, van Marken Lichtenbelt WD. Brown adipose tissue in morbidly obese subjects. PLoS One. 2011 Feb 24;6:1-6.

Schreinemacher MH, Vijgen GH, Dagnelie PC, Huizinga BF, Bloemen JG, Bouvy ND. Incisional hernias in old stoma wounds: a cohort study. Archives of Surgery. 2011 Jan;146:94-9. 


\section{Submitted}

Vijgen GH, Bouvy ND, Majoie M, Rijkers K, Cornips E, Leenen L, Brans B, van Marken Lichtenbelt WD. Brown adipose tissue after vagus nerve stimulation. Submitted.

Vijgen GH, Sparks LM, Bouvy ND, Schaart G, Hoeks J, Schrauwen P, van Marken Lichtenbelt WD. Mitochondrial respiratory analysis of human brown adipose tissue. Submitted.

Vijgen GH, Bouvy ND, Hoeks J, Schrauwen P, van Marken Lichtenbelt WD.

Skeletal muscle mitochondrial function before and after bariatric surgery. Submitted.

van der Lans AJJ, Hoeks J, Brans B, Vijgen GH, Visser M, Vosselman MJ, Jörgensen J, Wu J, Spiegelman B, Mottaghy F, Schrauwen P and van Marken Lichtenbelt WD. Cold acclimation revruits brown adipose tissue and increases non-shivering thermogenesis in humans. Submitted.

\section{Grants and awards}

2011 Collegium Chirurgicum Neerlandicum Travel Grant

2010 Kootstra Talent Fellowship 

Curriculum Vitae 


\section{About the author}

Guy Vijgen was born on the 13th of June 1985 in Geleen, the Netherlands. In 2003 he finished secondary school at the Graaf Huyn College in Geleen. Consecutively he started his medical training at the Maastricht University Medical Center. After graduating medical school in $\mathbf{2 0 0 9}$ he started working in a combined function as a resident in bariatric surgery and $\mathrm{PhD}$-student at the Department of Surgery in the Maastricht University Medical Center under the supervision of dr. N.D. Bouvy, dr. W.D. van Marken Lichtenbelt and prof. dr. P. Schrauwen. After obtaining a Kootstra Talent Fellowship in 2010 he became a fulltime PhD-student at the Department of Human Biology and the Department of Surgery. In July 2012, he started as a resident in the Department of General Surgery at the Sint Franciscus Gasthuis in Rotterdam under supervision of dr. G.H.H. Mannaerts. 
